\title{
The eye as a miRror
}

Citation for published version (APA):

Roblain, Q. (2021). The eye as a miRror: targeting microRNAs in ocular pathologies. [Doctoral Thesis, Maastricht University, University of Liège]. Maastricht University. https://doi.org/10.26481/dis.20210315qr

\section{Document status and date:}

Published: 01/01/2021

DOI:

10.26481/dis.20210315qr

Document Version:

Publisher's PDF, also known as Version of record

\section{Please check the document version of this publication:}

- A submitted manuscript is the version of the article upon submission and before peer-review. There can be important differences between the submitted version and the official published version of record.

People interested in the research are advised to contact the author for the final version of the publication, or visit the DOI to the publisher's website.

- The final author version and the galley proof are versions of the publication after peer review.

- The final published version features the final layout of the paper including the volume, issue and page numbers.

Link to publication

\footnotetext{
General rights rights.

- You may freely distribute the URL identifying the publication in the public portal. please follow below link for the End User Agreement:

www.umlib.nl/taverne-license

Take down policy

If you believe that this document breaches copyright please contact us at:

repository@maastrichtuniversity.nl

providing details and we will investigate your claim.
}

Copyright and moral rights for the publications made accessible in the public portal are retained by the authors and/or other copyright owners and it is a condition of accessing publications that users recognise and abide by the legal requirements associated with these

- Users may download and print one copy of any publication from the public portal for the purpose of private study or research.

- You may not further distribute the material or use it for any profit-making activity or commercial gain

If the publication is distributed under the terms of Article $25 \mathrm{fa}$ of the Dutch Copyright Act, indicated by the "Taverne" license above, 


\section{The eye as a miRror: targeting microRNAs in ocular pathologies}

Quentin Roblain 
The research described in this thesis was supported by a joined $\mathrm{PhD}$ funding program in the framework of the cooperation between the University of Liège and Maastricht University named "Towards a joint imaging valley".

This submitted dissertation contains unpublished confidential data. Members of the jury and anyone receiving this thesis manuscript are therefore bound to respect confidentiality and non-disclosure of the reported data to third parties by printout, electronic copy, and/or any other manner of communication.

Printing: Intercopy (Sart-Tilman, Liège)

Copyright () 2021 Quentin Roblain (roblain.quentin@gmail.com)

Cover design by: Samuel Buisseret 


\section{Maastricht \\ University}

\section{The eye as a miRror: targeting microRNAs in ocular pathologies}

\section{Dissertation}

To obtain the degree of Doctor in Biomedical and Pharmaceutical Sciences at the University of Liège and the degree of Doctor at Maastricht University, on the authority of the Rector Magnifici Prof. dr. Pierre Wolper and Prof. dr. Rianne M. Letschert in accordance with the decision of the Board of the Deans, to be defended in public on Monday $15^{\text {th }}$ of March 2021 at 13:00 hours

\section{Quentin Roblain}

Born in Liège, Belgium, on March $31^{\text {st }} 1992$ 


\section{Supervisors:}

Prof. Dr. Agnès Noël - University of Liège, Belgium

Prof. Dr. Stephane Heymans - Maastricht University, The Netherlands

\section{Co-supervisors:}

Dr. Julie Lecomte - University of Liège

Dr. Vincenza Caolo - Maastricht University

\section{Assessment committee:}

Prof. Dr. Paula da Costa Martins, Maastricht University, Chair assessment committee Prof. Dr. Lieve Moons, KULeuven, Belgium

Prof. Dr. Bernard Duchesne, University of Liège

Dr. Bernard Mari, Côte d'Azur University, France

Dr. Ingrid Struman, University of Liège

Dr. Theo Gorgels, Maastricht University

Dr. Sebastien Foulquier, Maastricht University 


\section{Table of content}

Preface

Chapter 1: MicroRNAs in the eye, key concepts 13

Chapter 2: Resistance to Retinopathy Development in Obese, Diabetic and Hypertensive ZSF1 rats: an exciting model to identify protective genes 55 Chapter 3: MicroRNA expression profile in diabetic patients and Akimba mice

Chapter 4: Intravitreal injection of anti-miRs against miR-142-3p reduces angiogenesis and microglia activation in a mouse model of laser-induced choroidal neovascularization 101

Chapter 5: General Discussion 127

Addendum 137 


\section{Preface}


Vision is our most essential sense and plays a critical role in every stages of our life. Everyone, if they live long enough, will experience at least one eye condition during their lifetime. While some eye conditions do not typically cause vision impairment, such as eyelid inflammation, conjunctivitis or dry eye syndrome, others can cause severe visual defects. Among these deleterious eye conditions, uncorrected refractive error, cataract, age-related macular degeneration (AMD), glaucoma, and diabetic retinopathy (DR) are the major causes of vision impairment worldwide [1]. Globally, it is estimated that at least 2.2 billion people live with a vision impairment, in which for 1 billion of these cases, vision impairment could have been prevented or has yet to be addressed [2]. Humans are fairly unique in their reliance on sight as the dominant of the five senses, since most of the information about our surrounding environment is gained thanks to the eyes. Eye conditions, despite causing no death, can thus dramatically affect life quality of patients, both at the professional and personal sides.

Anti-angiogenic drugs emergence resulted in great and unprecedented advances in AMD and DR clinical management during the past decades [3]. Local administrations of antiangiogenic molecules now allow possible disease progression control. However, these drugs show efficiency limitations in disease control and still, no cure for AMD and DR are available. Thus, the development of new innovative molecules is needed to overcome this unmet clinical need. MicroRNAs are recently arose non-coding RNAs whose implications have been demonstrated in a large panel of pathological conditions. These short RNA molecules emerge thus as promising candidates for drug development. In recent years, several clinical trials started to evaluate microRNA inhibition or overexpression strategy in pathologies such as cancer, wound healing and viral infection $[4,5]$. To date, microRNA as therapeutic target in the context of ocular diseases has not yet been implemented in clinical trial. 


\section{Aims and outline of this thesis}

This project is the result of a collaborative work between Maastricht and Liège universities. The aims of this thesis are: 1) to evaluate the potential of ZSF1 rat as a model of diabetic retinopathy, 2) to identify dysregulated microRNAs in animal models of DR and AMD and 3) to explore whether the modulation of promising microRNA candidates identified under 2) is able to influence choroidal neovascularization.

In Chapter 1 - MicroRNAs in the eye, key concepts -, we introduce, review and summarize the current knowledge on every concept needed for a comprehensive reading of subsequent chapters, microRNAs being the common thread between sections. A focus is made on their biology, their implication in the developing and adult eye, as well as in the pathological eye and finally, their role in microglia cells.

The results section is subdivided in the 3 following chapters:

In Chapter 2 - Resistance to Retinopathy Development in Obese, Diabetic and Hypertensive ZSF1 rats: an exciting model to identify protective genes -, we demonstrate that the ZSF1 rat model, despite showing hyperglycemia and hypertension, failed to develop diabetic retinopathy. We next highlight a potential gene network that could prevent ZSF1 rats from developing DR.

In Chapter 3 - MicroRNAs expression profile in diabetic patients and Akimba mice -, we reveal an expression profile of microRNAs in human and mouse samples and discuss their potential as biomarkers or therapeutic targets in DR.

In Chapter 4 - Intravitreal injection of anti-miRs against miR-142-3p reduces angiogenesis and microglia activation in a mouse model of laser-induced choroidal neovascularization -, we provide experimental evidence that miR-142-3p is specifically overexpressed in CNV lesion in a mouse model of choroidal neovascularization. We next demonstrate that this microRNA is able to activate microglia cells both in vitro and in vivo.

Finally, in Chapter 5 - General discussion -, we set our results in the general context of biomedical research and provide perspectives for future investigations. 


\section{References}

1. Flaxman, S.R., et al., Global causes of blindness and distance vision impairment 1990-2020: a systematic review and meta-analysis. The Lancet Global Health, 2017. 5(12): p. e1221-e1234.

2. $\quad$ WHO, World report on vision. 2019.

3. Kim, L.A. and P.A. D'Amore, $A$ brief history of anti-VEGF for the treatment of ocular angiogenesis. Am J Pathol, 2012. 181(2): p. 376-9.

4. Jones, D., Setbacks shadow microRNA therapies in the clinic. Nat Biotechnol, 2018. 36(10): p. 909-910.

5. Hanna, J., G.S. Hossain, and J. Kocerha, The Potential for microRNA Therapeutics and Clinical Research. Front Genet, 2019. 10: p. 478. 
Chapter 1: MicroRNAs in the eye, key concepts 


\section{Introduction}

MicroRNAs (miRNAs or miRs) are cross-kingdom short non-coding RNAs about 2024 nucleotides in length that are involved in post-transcriptional regulation of gene expression in animals, plants and microbes [1]. First discovered in the worm Caenorhabditis elegans during the year 1993 by Lee and colleagues [2], microRNAs are now known to be expressed by viruses, bacteria, algae, plants, fungi and animals, including humans [3]. The number of mature miRNAs identified in human is currently 2693 (miRbase, July 2020), many of them being expressed in a tissue-dependent or time-dependent manner. By regulating over than $60 \%$ of gene expression, it is well established that human miRNAs play fundamental roles in both development and adult life, as well as in healthy and pathological states [4, 5]. Non-coding RNA dysregulation, including microRNAs, is associated with cancer [6], cardiovascular diseases [7] and many ocular diseases, as supported by a growing body of evidences concerning microRNA complications in eye disorders [8,9]. This chapter aims at introducing each concept that will be used and discussed in the present manuscript, using microRNAs as a guideline. Thus, a brief summary of basic aspects of microRNA biology, with a particular focus on miR-142 is first proposed. In a second time, basic aspects of age-related macular degeneration (AMD) and diabetic retinopathy (DR) as well as their regulation by microRNAs will be described. Finally, a focus on microglia, which have been part of this work, will be presented.

\section{MicroRNA biology}

\section{- MicroRNA biogenesis}

Mature, and thus functional, microRNAs are processed from precursor RNA molecules called primary or pri-miRNA, which are transcribed by RNA polymerase II from miRNA genes (canonical miRNAs) (Figure 1) [10,11]. Each canonical pri-miRNA is a substrate for Microprocessor, a heterotrimeric complex made of Drosha endonuclease and its partner protein DGCR8. Each stem of a pri-miRNA is cut by one of the two RNase III domain of Drosha, liberating thus a $\sim 60$ nucleotides stem-loop called pre-miRNA. Of note, noncanonical miRNAs bypass Microprocessor and are directly transcribed into a pre-miRNA from protein-coding gene introns. After export from the nucleus to the cytoplasm through Exportin 5 and Ran-GTP, hairpin pre-miRNAs resulting from both canonical and noncanonical undergo further processing by Dicer, another endonuclease with two RNase III domains. Dicer and its partner protein TRBP cut both strands near the loop to generate the miRNA duplex, a 20-23 nucleotides double stranded RNA molecule. One strand of this duplex will constitute the mature miRNA while the other strand is its passenger strand (miRNA*). Once formed, the miRNA duplex is loaded into an Argonaute (AGO) protein assisted from chaperone proteins in an ATP-dependent manner. At that time, passenger strand is expulsed from the duplex because of its less adapted 5' terminus to bind to Argonaute, and the mature silencing complex is formed with the mature miRNA. Once 
loaded into the silencing complex, the mature miRNA pairs to complementary sites within mRNA and other transcripts to express their post-transcriptional regulation. Interestingly, once loaded into the silencing complex, most miRNAs are more stable than mRNAs, exhibiting half-life of days [12].

Of note, miRNA* strands are not systematically degraded [13], and can also be loaded in silencing complex. Since 2012, a novel classification of miRNAs has been made, replacing the old miRNA/miRNA* nomenclature by more accurate miRNA-3p/miRNA-5p one (Figure 2). For most of the microRNAs, both $3 p$ and $5 p$ arms have a biological function and have distinct independent expression pattern and mRNA targets [14, 15].

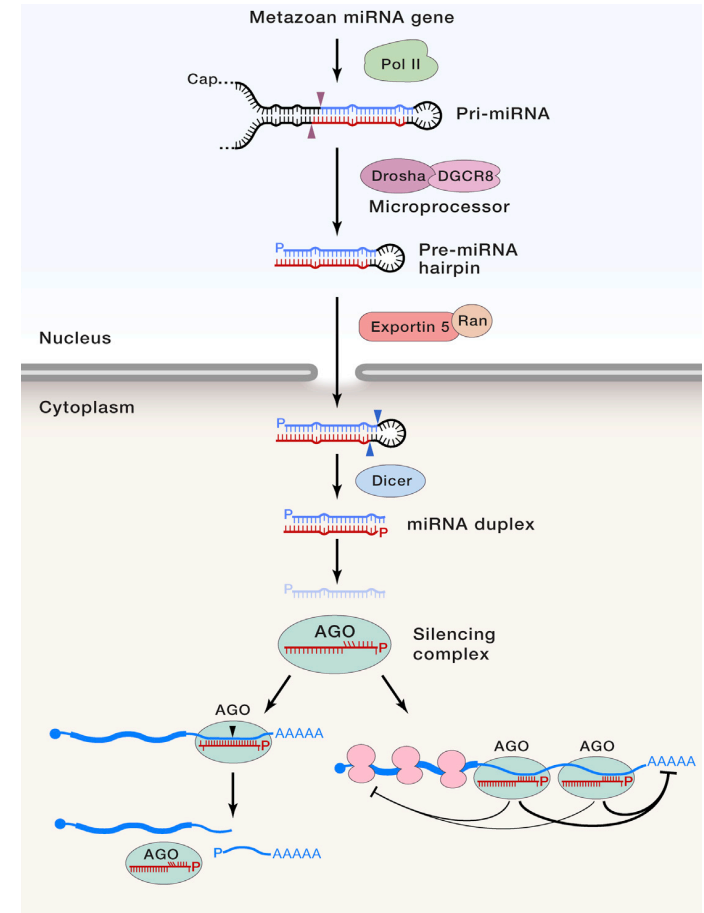

Figure 1. Metazoan microRNA biogenesis and function

Pri-miRNA are transcribed by RNA Pol II from miRNA gene and are then cleaved by Microprocessor, a heterotrimeric complex composed of Drosha and DGCR8. The resulting pre-miRNA hairpin is next exported from the nucleus to the cytoplasm via Exportin 5 and Ran-GTP. In the nucleus, pre-miRNA are further processed by Dicer to form a miRNA duplex. This duplex is loaded into an AGO protein and the passenger strand (in blue) is released, forming thus the silencing complex. This silencing complex finally pairs to complementary sites within the 3' UTR region of target mRNA and expresses its post-transcriptional repression by either mRNA destabilization or translational repression.

Adapted from Bartel (2018) 


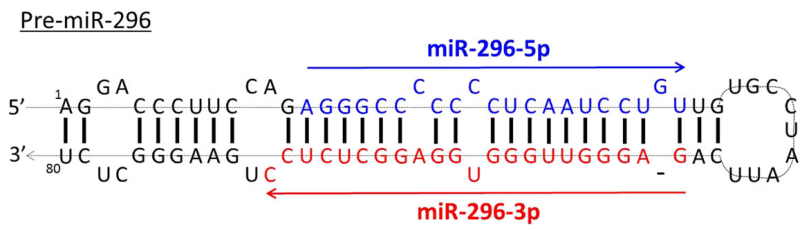

$\underline{\operatorname{miR}-296}$

$\begin{array}{lll}\text { miR-296-5p } & \text { 5' - AGGGCCCCCCCUCAAUCCUGU - 3' } & \text { Lenth: 21nt } \\ \text { miR-296-3p } & \text { 5' - GAGGGUUGGGUGGAGGCUCUCC - 3' } & \text { Lenth: } 22 n t\end{array}$

Figure 2. MicroRNA-5p and microRNA-3p derived from the same pre-miR Structure of the pro-angiogenic and pro-inflammatory miR-296, both miR-296-5p and miR-296-3p being produced from pre-miR-296.

Adapted from $\mathrm{Li}(2018)$

\section{- MicroRNA regulatory mechanisms}

MicroRNAs recognize and repress their target transcripts through two alternative modes, depending on pairing coverage. If pairing between the microRNA and its mRNA target is sufficiently extensive, and if the microRNA is associated with an AGO protein, then the miRNA directs slicing of the target [16]. This first repression mode is common for plant miRNAs [17] but only a few cases of direct slicing have been reported in human [18]. The repression mode that dominates in human is slicing-independent and does not require an extensive pairing coverage. Instead, it requires an adaptor protein, TNRC6, recruited by AGO, interacting with the poly(A)-binding protein PABPC associated with the mRNA poly(A) tail and recruiting itself a deadenylaze complex [19]. This latest complex shortens the poly(A) tail, which causes mRNA destabilization through decapping and 5'-to-3' exonucleolytic decay. Besides transcriptional repression, TNRC6 recruitment also causes the mRNA to be less efficiently translated due to inhibition of translation initiation. The relative importance of TNRC6-mediated transcriptional or translational repression depends on the developmental context of the cell, mRNA transcriptional repression dominating in postembryonic cells. Thus, in mammals, regardless the miRNA, cell type or growth condition, mRNA destabilization explains $66-90 \%$ of miRNA-mediated repression [20].

Transcriptional repression of mRNA target mediated by TNRC6 is triggered by WatsonCrick pairing between the miRNA seed sequence (miRNA nucleotides 2-8) and sites within the 3'-UTRs of target mRNAs [21]. Thus, it is possible to predict putative targets by computational methods [22]. Most of these methods rely on algorithms implemented with information such as but not limited to the miRNA sequence, the 3'UTR of mRNA, the seed sequence, thermodynamics parameters, the miRNA 3' site and the conservation status of both miRNA and mRNA sequence [23]. Bioinformatics has thus become a powerful tool for algorithmic target prediction with online free databases such as TargetScan, miRTarBase and 
miRDB [22, 24, 25]. However, experimental confirmation using luciferase reporter assays remain the golden standard for a biological validation of predicted mRNA targets.

\section{- Regulation of microRNA expression}

While regulating gene expression, microRNAs themselves are also subject to a tight temporal and spatial expression and production control. MicroRNAs processed from the introns of mRNAs are under the same transcriptional regulation as the mRNA of their host gene [26]. All other canonical miRNA loci are also under RNA Pol II transcriptional control, indistinguishable from that of protein-coding gene [27]. Once the miRNA locus has been transcribed, diverse regulatory factors can influence either the stability or processing of individual hairpin molecule. In addition, multiple regulatory mechanisms influence the accumulation and activity of both Microprocessor and Dicer [28].

MicroRNA abundance and stability are also regulated by miRNA decay, but it has not extensively been studied so far. A target-directed miRNA degradation mechanism (TDMD) has been suggested [29]. In this mechanism, the complementary RNA target promotes degradation of its miRNA, accompanied by post-transcriptional modification of the miRNA sequence, i.e. tailing (addition of nucleotides at the 3' end) and trimming (shortening), and unloading from AGO [30]. While some miRNAs show half-life of days once associated to AGO [12], other miRNAs are not so stable. For example, some neuronal miRNAs have variable stability and a few are constitutively unstable, enabling a more rapid response to transcriptional changes [31]. Triggering of TDMD mechanism by different features could explain some of the differences observed in endogenous miRNA stability [32].

\section{- MicroRNA impact on gene regulation and biological functions}

MicroRNAs are considered as fine-tuners of gene expression rather than on-off regulators [33]. A single mature microRNA species may potentially target hundreds of mRNA molecules. Therefore, dysregulated miRNA expression can disrupt RNA networks that are otherwise tightly regulated. However, while repressing hundreds of target mRNAs, protein level of miRNA targeted gene are only slightly influenced by the modulation of microRNA expression as revealed by proteomic analysis [34]. MicroRNAs thus act through an assembly of slight perturbations which may be a favorable aspect for their use as therapeutic targets. This "one hit - multiple targets" appeared to be a promising therapeutic concept but this inherent particularity of microRNA complicate the identification of microRNA targets that explain the observed phenotype [35]. While some microRNA target sets are enriched in specific cellular function such as the oncogenic miR-17 92 cluster with enriched targets enrolled in growth control [36], most of microRNAs seem to influence diverse biological functions. In the same way, many specific biological functions are regulated by several microRNAs simultaneously. The field of microRNAs biological function study has been explored via the use of knock-out and mutant animals, including nematode, fly and mouse. D. P. Bartel extensively reviewed all current literature about metazoans microRNAs [10]. Thus, many microRNAs are required for proper development of skeleton, teeth, brain, eyes, neurons, muscle, heart, lungs, kidneys, vasculature, liver, 
pancreas, intestine, skin, fat, breast, ovaries, testes, placenta, thymus and each hematopoietic lineage. MicroRNAs are also important for many cellular functions and physiological processes.

These functional particularities of microRNAs lead to many successes in determining their general mechanisms of function, but microRNA application in translational area has not had much success yet [37]. In 2019, only 7 microRNAs (miR-16, miR-21, miR-29, miR-34, miR-92, miR-122 and miR-155) were therapeutic candidates in clinical development, with 3 reaching phase II and the other ones still in phase I [38, 39].

\section{- Experimental microRNA inhibition and overexpression}

MicroRNA functions have been largely described through the use of gain or loss of function strategies. While different genetic knock-out or knock-in animal models have been implemented to decipher microRNA functions, the use of different kind of inhibitors or enhancers of microRNA expression is also extensively used in experimental work [40] (Figure 3).

On one hand, microRNA inhibition can be performed through the use of different molecules such as but not limited to antagomiR, LNA (Locked Nucleic Acid) antimiR and miR mask [41]. AntagomiRs are single-stranded RNA molecules conjugated with cholesterol, either at the 3' or 5' terminus, and 21-23 nucleotides in length. Complementary to their target miRNA, they bind to it thus preventing the miR-mediated repression of the target mRNA. Once intercepted by the antagomiR, miRNA is either degraded or stabilized in a miR-antagomiR duplex. LNA antimiRs present a methylene bridge between the 2'-O and the 4'-C atoms, locking thus the ribose ring. This chemical enhancement results in an increased potency, safety and binding affinity [40]. LNA antimiRs are shorter than antagomiRs, covering miRNA seed sequence and are made of either single stranded RNA or DNA molecules. Finally, miR-masks are single stranded 2'-O-methyl modified antisense oligonucleotides complementary to miR target sequence on mRNA 3'UTR. These oligonucleotides thus prevent the miRNA binding to a specific target gene.

On the other hand, microRNA overexpression is possible through the use of different expression systems. Thus, conventional pri-miR or pre-miR are available, as long with mature miR expression systems [42]. Mature miR expression systems, often referred as to miR mimics, are chemically synthetized double-stranded RNA that can easily transfected in host cells.

At the experimental level, assessing the efficiency of miRNA inhibition and overexpression can sometimes be tricky. Indeed, microRNA inhibitors do not always induce the degradation of their miRNA targets, also possibly protecting them from cellular degradation. For both inhibitor and enhancer molecules, effect on the mRNA can also be different, depending on the miRNA itself. Assessing miRNA inhibition and overexpression should be thus done by evaluating the level of miRNA and mRNA, at both the transcript and protein levels, in the different experimental conditions. 


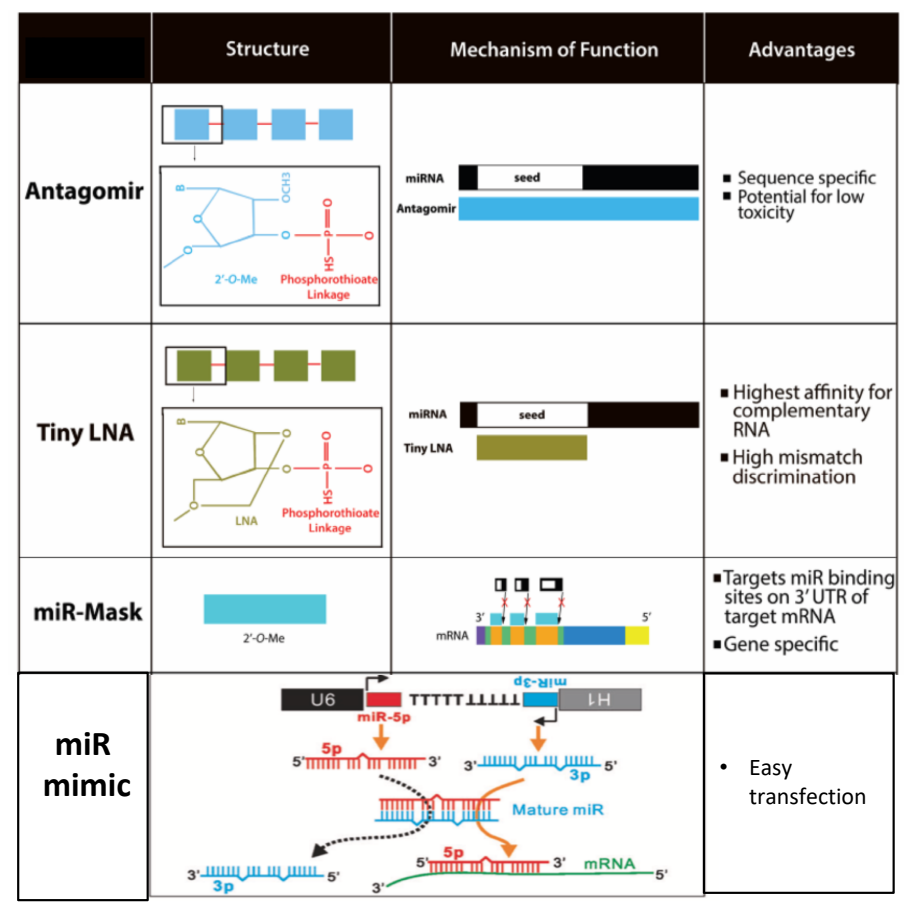

\section{Figure 3. Experimental miRNA inhibition and overexpression}

While miRNA inhibition can be performed through the use of antagomiRs, LNA antimiRs or miR-masks, miRNA overexpression is possible through the use of miRNA mimics. AntagomiRs and LNA antimiRs bind to the full miRNA sequence and only to its seed sequence, respectively. MiR-mask directly bind to mRNA sequence targeted by specific miRNA. Mature miRNA mimic are synthetic molecule "mimicking" endogenous miRNA.

Adapted from Dang (2015) and Fan (2019) 
- Focus on miR-142:

In the subsequent section, a focus on miR-142 will be made since this specific microRNA is the key component of Chapter 4 and part of Chapter 3. With only 13 hits on PubMed in 2009 with the key word "miR-142", microRNA-142 was part of 189 publication titles in 2020. In 10 years, this specific microRNA has been studied in a large context of diseases and its roles in both physiological and pathological conditions only start to be elucidated, emerging as a critical regulator of many biological processes.

MiR-142 gene is encoded in chromosome 17 in human and in chromosome 11 in mouse. In both species, miR-142 gene is an intronic microRNA located a few kb upstream TSPOAP1, a gene encoding for a benzodiazepine receptor associated protein (NCBI genomic database, July 2020). It is associated in a cluster with miR-4736, a poorly described microRNA. Interestingly, human, mouse and rat versions of mature miR-142-3p and miR142-5p are fully conserved, suggesting recurrent functions and allowing possible translation of rodent to human results. Mature miR-142-3p is a 23-base long oligonucleotide with a $35 \%$ GC content, generated from an 87-nucleotide long hairpin in human and a 64-nucleotide long hairpin in mouse. Mature miR-142-5p is two nucleotides shorter with 33\% GC content. (miRBase, July 2020) (Figure 4). 
A

\section{Human chromosome 17 / Mouse chromosome 11}

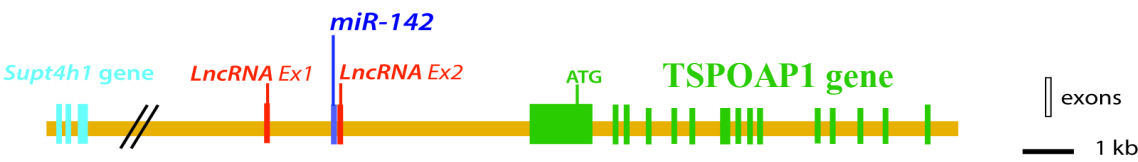

B

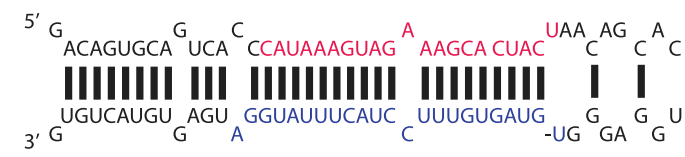

C

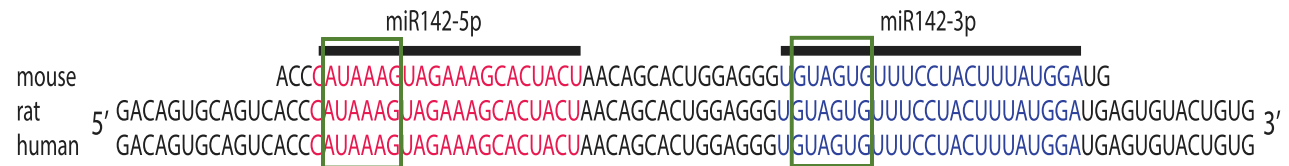

Figure 4. Genomic location and sequences of miR-142 hairpin and mature microRNA A. MiR-142 gene is located within chromosome 17 in human and chromosome 11 in mouse, a few kb upstream TSPOAP1 gene and in the vicinity of an exon belonging to a lncRNA.

B. Stem-loop structure of the hairpin pre-miRNA-142 containing both miR-142-5p sequence (in red) and miR$142-3 p$ (in blue).

C. Alignment of mouse, rat and human pre-miR-142, showing fully conserved sequences and seed sequences (green boxes).

Adapted from Shrestha et al. (2015) and Sharma (2017) 
MiR-142 exhibits functions in development, homeostasis and disease, with a particular role in hematopoietic system regulation [43]. It was first identified in hematopoietic stem cells by Chen and colleagues [44] and was than further described for its critical role in zebrafish during the formation and differentiation of hematopoietic stem cells and progenitor lineages [45, 46]. Two teams have generated either miR-142 knockdown (KD) or knockout (KO) mice to further unveil miR-142 biological function in vivo. First, Chapnick and colleagues developed a miR-142 KD mouse strain, using exogenous gene trap technology [47]. They reported a critical role of miR-142 during megakaryopoiesis. MiR-142 KD caused impaired megakaryocyte maturation, inhibition of polyploidization, abnormal platelet formation and thrombocytopenia. Platelet formation was altered due to a poorly controlled actin filament homeostasis in the absence of miR-142. One year after Chapnick, Shrestha and team generated miR-142-null mice using homologous recombination [48]. Embryonic miR-142 null mice exhibit increased $W n t$ signaling in lungs while pups were born alive and were normally represented, indicating an absence of any embryonic lethality. Adult miR142-null animals were viable and displayed impaired hematopoietic lineage formation. Taken together, Chapnick and Shrestha's results strongly argue for a critical function of miR142 during hematopoiesis. However, both KD and KO mice generated exhibit either a strong decrease or an extinction of both miR-142-3p and miR-142-5p, making an impossible distinction between each strand specific functions. Later on, several new functions have been described for both miR-142 mature forms. Thus, evidences suggest a possible mechanism by which miR-142 may play an important role in maintaining stem cell pluripotency during organogenesis [49]. In addition of keeping a bimodal state in stem cells, miR-142 seems to be implicated during lung development by decreasing proliferation of mesenchymal cells and premature differentiation of smooth muscle progenitor cells [50]. Besides its role during development, miR-142 is also downregulated during cardiac hypertrophy, a normal mechanism possibly resulting in heart failure under hypertension conditions [51]. MiR-142 also exhibits several functions related to immune response and immune tolerance. Thus, a high level of miR-142-3p was observed in the peripheral blood mononuclear cells from immune tolerant renal transplanted patients [52]. Furthermore, both miR-142 mature forms are associated with several cancer types. Thus, miR-142-3p level in cervical cancer epithelial cells was significantly lower than in healthy cervical epithelial cells, indicating a regulatory impact of miR-142-3p in cervical cancer [53]. MiR-142-3p was also found to inhibit tumor progression and invasion in hepatocellular carcinoma cells [54]. On the other hand, miR$142-5 \mathrm{p}$ overexpression correlates with cancer progression by suppressing growth inhibition of cancer cells [55]. Thus, miR-142 implication in cancer is thus poorly understood and seems to be cancer-type specific. Finally, recent publications focusing particularly on miR$142-3 p$ show that this specific microRNA is associated with a wide range of inflammation and neovascularization associated diseases such as breast cancer [56], neuroinflammation [57], gestational diabetes mellitus [58] and uveal melanoma [59]. Also, miR-142-3p expression was found to be upregulated in a mouse model of retinal degeneration [60]. It is now clear that both miR-142-3p and miR-142-5p are critical regulators of several physiological process, mainly hematopoiesis and play a role in a wide variety of diseases. 
Because of their status of recently described microRNAs, miR-142-3p and miR-142-5p biological functions are currently emerging. In regard of the growing number of publications concerning miR-142, one can hypothesize that this microRNA has probably still many unknown functions. However, it is now clear that both mature microRNAs have distinct but also shared properties. A deeper analysis of current literature reveals that mRNA targets of miR-142 are not well described. Indeed, compared to well-studied microRNAs such as miR155 targeting SOCS1 mRNA and miR-146a targeting IRAK1 and TRAF6 mRNAs, both mature forms of miR-142 are still lacking conserved targets that could be studied in a wide range of cell types and tissue types. Thus, human miR-142-3p most cited targets in the literature are ARNTL and RAC1 (miRTarBase, July 2020). Further characterization of mRNA targets will be needed for a better understanding of miR-142 regulatory mechanisms. 


\section{MicroRNA in the developing and adult eye}

The eye is a complex and specialized sense organ responsible of the conversion of a light signal into electro-chemical impulses through the nervous system. Despite structural differences through species, molecular biology has now provided supportive evidences suggesting that the eye has arisen a single time during evolution. Thus, the involvement of highly conserved transcription factors such as PAX6 in the genetic control of eye development in organisms ranging from planarians to human, strongly argue for a monophyletic origin of the eye [61]. Eye development is dictated by conserved genes throughout the animal kingdom, giving rise to diversified ocular structure, from basic forms such as pit eyes to near-to-perfect human eye [62]. The human eye is made up of three coats, which enclose the optically clear aqueous humor, lens and vitreous body. The outermost coat consists of the cornea and the sclera; the middle coat contains the main blood supply to the eye and consists, from the back forward, of the choroid, the ciliary body, and the iris. The innermost layer is the retina, lying on the choroid and receiving most of its nourishment being derived from the retinal vessels (Figure 5A). The retina is a complex nervous structure, being an outgrowth of the forebrain. It is made of specialized cells such as retinal pigmented epithelial (RPE) cells, photoreceptors (i.e. rod and cone cells), bipolar neurons and ganglion cells but it also contains more generic cells such as endothelial cells and inflammatory cells (including microglia cell) (Figure 5B). Of note, most of the eye components are free from lymphatic vasculature excepted for corneal limbus, ciliary bodies and lacrimal glands. Despite lymphangiogenesis is known to play important role during corneal transplantation and ocular tumor progression, its importance in most eye diseases is currently unknown [63].

Because vertebrate eye development is tightly regulated by a specific gene expression pattern, it is not surprising that microRNAs are major actors during ocular organogenesis. While deletion of miR-302 in mice results in a late embryonic lethal phenotype, abnormal eye development can be observed in miR-302 null mice from E13.5, suggesting a critical role of this specific microRNA in ocular organogenesis [64]. The miR-183/96/182 cluster is a sensory organ specific microRNA, known to regulate maturation of sensory organs and diseases such as cancer and autoimmune disorders [65]. This cluster is highly expressed in mouse retina and its inactivation results in early-onset and progressive synaptic defects of the photoreceptors and progressive retinal degeneration [66]. Further studies demonstrated that ablation of only two of the three members of the cluster, miR-183 and miR-96, significantly affects photoreceptor maturation and maintenance [67]. Other microRNAs such as miR-124a and miR-211 have been identified as critical regulators of cone photoreceptor function and survival, leading to altered visual function when deleted [68, 69].

Besides their role during eye development and photoreceptor maturation, microRNAs are also important for eye homeostasis by regulating major eye specific cell types physiology such as photoreceptors, RPE cells, ganglion cells and keratocytes [70]. MicroRNAs implication in eye homeostasis is further confirmed by the huge population of microRNAs expressed in human and mouse eye as revealed by deep sequencing analysis. Thus, Soundara and colleagues showed that 320 different microRNAs are expressed in mouse retina, while 
choroid/RPE complex expresses 340 microRNAs [71]. In a similar way, Karali demonstrated an even greater number of microRNAs expressed in human retina and choroid/RPE complex, with 480 and 416 detected microRNAs, respectively [72]. In mouse, a microRNA expression atlas of the eye (miRNeye project) was made by a combination of microarray analysis and RNA in situ hybridization, providing insights into microRNA profile at the tissue level in WT mice (embryonic stage E16.5 and post-natal days P0, P8 and P60) [73]. This atlas reveals that microRNAs are expressed in all eye tissues, with specific or not expression patterns during development and adult life. Concerning microRNA expression in the vitreous fluid, it is suggested that vitreal microRNAs may result from either retinal cell excretion (via exosomes or microvesicles) or from resident vitreal cells (phagocytes or hyalocytes - cells responsible for the production of structural vitreal molecules -) in non-pathological conditions [74]. Under pathological conditions, vitreal microRNAs may be produced by infiltrating immune cells or red blood cells (hemorrhagic condition). 


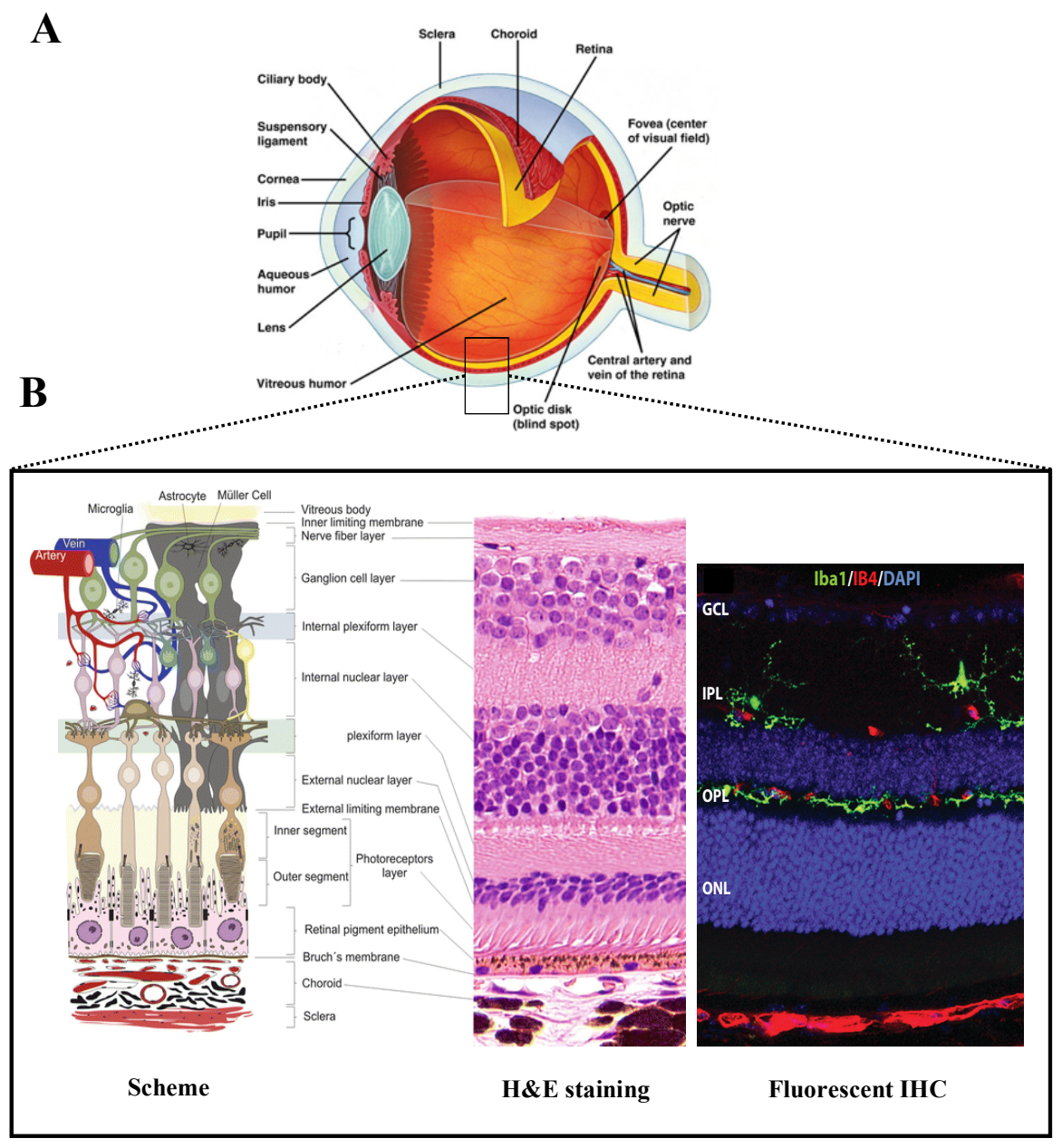

\section{Figure 5. Basic anatomy of the human eyeball and structure of its posterior pole}

A. The human eye is a near-to-spherical organ. The sclera is the external thick conjunctive tissue which at its most anterior region forms the cornea. The intraocular pressure is maintained thanks to the vitreous humor, a highly hydrated gel. The lens allows light refraction to the light-sensitive part of the eye, the retina. The choroid, lying between the sclera and the retina, is a vascular tissue responsible of retina maintenance.

B. The retina is a multilayer tissue composed of, from inner to outer region, inner limiting membrane (Müller cell endfeets), nerve fibre layer (ganglion cell axons), ganglion cell layer (GCL), internal plexiform layer (IPL, synapses between bipolar, amacrine and ganglion cells), internal nuclear layer (horizontal, bipolar and amacrine cell bodies and Müller cell nuclei), external plexiform layer (OPL, synapses between photoreceptors, bipolar and horizontal cells), external nuclear layer (ONL, photoreceptor cell bodies), external limiting membrane (junctions between Müller cells and photoreceptors), retina pigmented epithelium (RPE) and Bruch's membrane.

Microglia (Iba1 stained, in green) cell bodies are located within the IPL and OPL, with protrusions extending in adjacent layers, and are in close contact with blood vessels (IB4 stained).

Fluorescent immunohistochemical (IHC) section is adapted from Silverman and Wong (2018). 


\section{MicroRNA in the pathological eye}

As for many other organs, microRNAs are critical drivers of the correct development and homeostasis of the human eye but are also implicated in several ocular pathologies. Every component of the eye is subjected to microRNA regulation, possibly leading to disease if unproperly regulated. Retina, choroid, sclera, cornea, and lens are thus the major eye components that can be affected by microRNA dysregulation, leading to a variety of diseases such as age-related macular degeneration (AMD), diabetic retinopathy (DR), retinitis pigmentosa, refractive errors, cataract, uveitis, glaucoma and keratitis. In this section, a deeper analysis of pathophysiology of AMD and DR and their possible microRNAs dysregulation will be made.

\section{- $\quad$ Age-related macular degeneration and microRNAs}

\section{Epidemiology, etiology and animal models}

Age-related macular degeneration is a progressive retinal pathology affecting the elderly and is the fourth leading cause of blindness worldwide [75]. With 8,7\% of worldwide population currently affected by AMD, a substantial increase of this percentage is expected due to ageing population [76]. This pathology affects the macula, the central part of the retina responsible for high-acuity daylight and central vision [77]. Early and intermediate stages of AMD are characterized by the formation of sub-RPE deposits called drusen. These serve as precursor stages for the evolution of late AMD, which consists of two distinct phenotypes: (i) geographic atrophy, characterized by progressive degeneration of the RPE, photoreceptors and choriocapillaries, and (ii) neovascular AMD, characterized by the formation of choroidal neovascularization (CNV). These two late stages are highly sight-threatening, but wet, or neovascular, AMD prevalence is lower than the atrophic form and represents the most sightthreatening form. AMD etiology is complex and multifactorial, not currently fully understood. Main risk factors for developing AMD are increasing age, heredity and smoking [78]. CNV is the hallmark of wet AMD and occurs by breaking through the Bruch's membrane, invading the sub-retinal space and leaking fluids and bloods from newly immature hyperpermeable capillaries [79]. It is the final step of wet AMD and leads to severe visual loss [80]. Several animal models are available for studying wet AMD [81], but one of the most cited one in current literature is the laser-induced CNV mouse model [82]. This model relies on Bruch's membrane perforation via laser treatment, inducing subsequent choroidal neovascularization (Figure 6). 
Early pathological condition
Late neovascular pathological condition
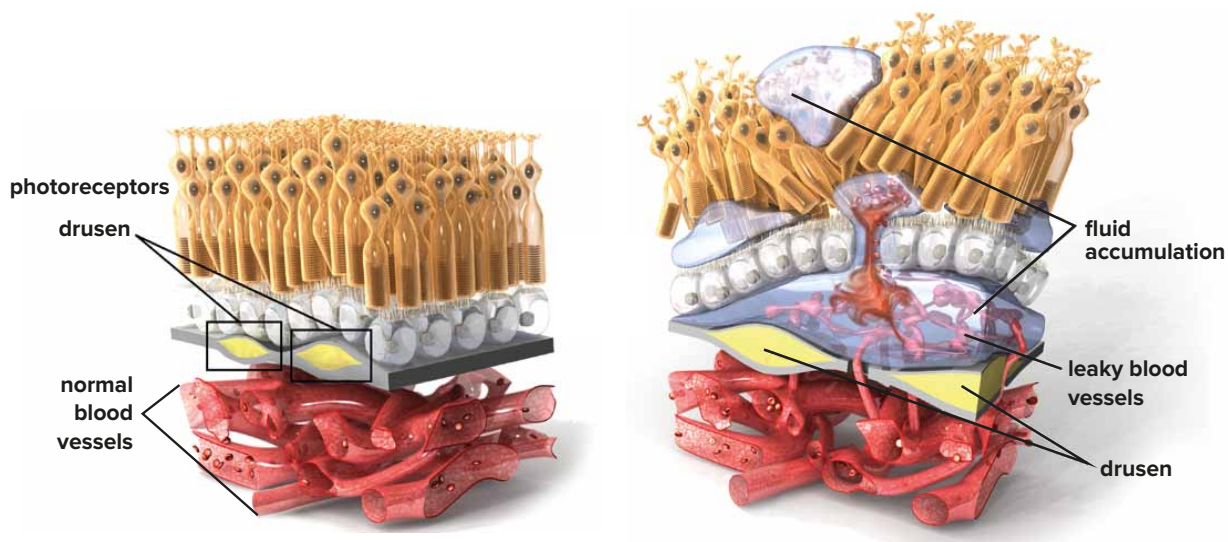

Figure 6. Choroidal neovascularization (CNV) is the hallmark of wet age-related macular degeneration

In early AMD, while photoreceptors and choroidal vessels remain unaltered, sub-RPE deposits called drusen are observed and can eventually trigger AMD progression. Choroidal neovascularization is the hallmark of late stage neovascular (or wet) AMD and is regulated by a balance of pro- and anti-angiogenic and inflammatory factors, resulting in the formation of leaky blood vessels. CNV in responsible of photoreceptors and RPE damage, thus threatening vision.

Adapted from The Angiogenesis Foundation (scienceofamd.org) 


\section{Pathophysiology}

AMD is a highly complex neurovascular disease, with a not fully described pathophysiology. Ageing, genetic components and environmental factors act together in the triggering of AMD, inducing angiogenesis and inflammatory pathways. CNV progression is tightly regulated by a balance of pro and anti-angiogenic factors, Vascular Endothelium Growth Factor (VEGF) being a key positive regulator of angiogenesis. Among all VEGF family members, VEGF-A and PIGF (Placental Growth Factor) have demonstrated functions during CNV progression, both stimulating neovascularization in animal models [83, 84]. Elevated levels of VEGF induce blood-retina barrier (BRB) breakdown, thus activating choroidal macrophage invasion of the retina. Other pro-angiogenic factors such as angiopoietin and HIF-1 (Hypoxia-inducible Factor-1) are also described for their contribution to $\mathrm{CNV}$ progression $[85,86]$. These pro-angiogenic factors stimulating $\mathrm{CNV}$ progression are counteracted by a series of anti-angiogenic factors. Among them, PEDF (Pigment Epithelium-Derived Factor) is expressed in balance with VEGF, and a decrease in its level is permissive for AMD in human [87]. Trombospodin-1 deficient mice subjected to laser-induced CNV exhibited exacerbation of neovascular surface, associated with an increased macrophage recruitment [88]. The contribution of other molecular mediators has been demonstrated in CNV progression, such as different members of the matrix metalloproteinase family (MMP-2, MMP-9 and MMP-13) and plasminogen activator inhibitor type-1 (PAI-1) [89-91].

Besides this tight regulation of pro and anti-angiogenic factors and even if AMD is not clinically considered as a classical inflammatory disease, many mechanisms of inflammation are involved during AMD onset [92]. The eye used to be considered as an immune privilege site. It is not sheltered from intraocular inflammation but has developed to limit it, so as to protect the delicate visual elements from damage that would be detrimental to visual acuity and therefore survival [93, 94]. Thus, the retina disposes of its own endogenous immunocompetent cells, microglia and dendritic cells, dealing with other eye immune cells such as macrophages and neutrophils. Production of inflammatory-related molecules, complement activation, recruitment of macrophages and microglia activation are thus the predominant mechanisms involved. Under normal aging conditions, the retina undergo a low-grade inflammation referred as parainflammation. Parainflammation is defined as a condition of tissue adaptive response to noxious stress or malfunction, and it has features which are considered as intermediate between normal/basal and inflammatory/acute states. While the physiological role of normal parainflammation is to preserve tissue homeostasis and to restore their function if exposed to prolonged stress, parainflammation is also implicated in both initiation and progression of many human age-related disorders, such as obesity, atherosclerosis and AMD $[95,96]$. Triggers of detrimental parainflammation in the retina are oxidative stress and oxidative species, oxidized lipoproteins, lipid peroxidation byproducts, advanced glycation end products (AGEs) and apoptotic cells [97]. Once triggered, detrimental parainflammation is responsible for complement system activation. The role of this innate immunity cascade reactions was initially suggested as a driver of wet AMD when complement components where found in drusen of AMD patients [98]. Since this discovery, 
many studies highlighted a highly significant correlation between AMD and polymorphism of gene encoding several molecules involved in complement pathway such as CFH, CFB and C2 [99, 100]. Although inflammatory processes involved during AMD are only partly elucidated, inflammation seems to be tightly associated with angiogenic processes during age-related macular degeneration progression.

\section{Clinical management}

Due to its highly complex and still not totally understood etiology, AMD was hardly treated until early 2000. First only treated by photodynamic therapy [101], neovascular AMD treatment has been largely enhanced during the last decades thanks to anti-VEGF therapy. Different anti-VEGF drugs emerged on the market, with efficiency improvement from generation to generation. The first anti-VEGF drug approved by the FDA was pegaptanib, a PEGylated short RNA oligonucleotide targeting VEGF $_{165}[102,103]$. A few years after pegaptanib, two antibodies, namely bevacizumab (Avastin ${ }^{\circledR}$ ) and ranibizumab (Lucentis ${ }^{\circledR}$ ), targeting all VEGF-A isoforms and their receptor-binding domain, respectively, emerged as the standard procedure for AMD treatment $[104,105]$. One of the latest anti-VEGF drug market-released is a dimeric recombinant fusion glycoprotein targeting a broad range of VEGF family members, aflibercept (Eylea $\left.{ }^{\circledR}\right)$ [106]. Finally, brolucizumab (Beovu $\left.{ }^{\circledR}\right)$ was FDA and EMA approved for the management of neovascular AMD in 2019 and 2020, respectively. Brolucizumab is a humanized single-chain variable fragment antibody inhibiting all VEGF-A isoforms, with a lower molecular mass when compared to other drugs, allowing thus a higher solubility and tissue-penetration [107, 108]. Unfortunately, despite this revolution of exudative AMD treatment, around one fifth of neovascular AMD patients are non-responsive or show a really poor response to anti-VEGF therapy [109], highlighting the need of an improvement of actual therapy. Of note, none drug is available at the moment for the treatment of geographic atrophy, the late stage of dry AMD. Several candidates are currently being tested in clinical trials, mainly focusing on either anti-inflammatory or neuroprotective therapy [110].

\section{MicroRNA implication}

The next big step in AMD treatment could eventually be achieved with microRNA therapy, but to reach this challenging goal it will imply a much deeper understanding of microRNA regulation of AMD. To date, several studies have focused on establishing microRNAs has potential biomarkers or therapeutic targets in AMD in both human and mouse study.

In human, all researches conducted are mainly profiling studies focused on determining a microRNA expression profile in human samples. Most of the studies focused on circulating microRNAs extracted from either whole blood [111, 112], plasma [113, 114] or serum [115, 116]. Circulating microRNAs are extracellular microRNAs, sometimes exosome encompassed, and recognized as an exciting new avenue of research for many pathologies [117, 118]. Unfortunately, these studies show great inconsistencies within the most dysregulated microRNAs and almost no replication is observed between these independent 
works. One can hypothesized that heterogenous patient cohorts and detection methods are the main factors that could explain these inconsistencies. Besides peripheral-blood circulating microRNAs, some studies have also focused on much more difficult to access samples. For example, Ménard and colleagues identified several neovascular AMD-specific microRNAs is the vitreous of diseased patients, with a reproducible expression pattern in plasma [114]. Thus, miR-146a-5p increased while miR-106b-5p and 152-3p decreased in the vitreous and plasma of wet AMD patients. Unfortunately, only miR-146a-5p overexpression was confirmed in other similar studies [115]. Pogue and colleagues showed an up-regulation of 7 inflammatory microRNAs, including miR-146a, in retinas of post-mortem AMD patients [119]. Interestingly, these microRNAs were also up-regulated in brains of postmortem Alzheimer disease patients, another neurogenerative disease sharing some features with AMD.

In rodent, both profiling and functional studies have been mainly done on mouse models of CNV. A recent study by Kiel and colleagues showed a microRNA expression profile of both circulating as well as retina and RPE-choroid complex tissue microRNAs in a laserinduced CNV mouse model [120]. Both miR-486a-5p and miR-92a-3p exhibited a decreased expression in blood while their expressions were higher in RPE-choroid complexes and not changed in retinas when compared to control condition. Interestingly, both microRNAs showed an impact on murine microglia cell viability in in vitro functional assays. In another similar study, expression patterns of microRNAs and transfer RNA-derived small RNAs (tsRNAs) from laser-induced CNV mouse model RPE-choroid-sclera complex were investigated [121]. Here the authors found that miR-10a-3p and miR-1247-5p were the most up and down regulated in $\mathrm{CNV}$ model, respectively. Unfortunately, no commonly dysregulated microRNAs were jointly highlighted in this study and Kiel's. Despite laserinduced $\mathrm{CNV}$ mouse model is the most frequent model, intravitreal injection of amyloid- $\beta$ (A- $\beta$ ) is sometimes used for A- $\beta$ elicited disease such as neurodegenerative disease and glaucoma $[122,123]$. This in vivo model of AMD has been designed on the basis of previous reports showing the presence of A- $\beta$ deposits in drusen of AMD patients [124, 125]. Romano and colleagues showed an up-regulation of miR-27a, miR-146a and miR-155 in retinas from A- $\beta$-elicited AMD rat [115]. It is not surprising to observe that both miR-146a and miR-155, two well-described pro-inflammatory microRNAs, are up-regulated in AMD rats. Concerning miR-155, divergent results have been found by two distinct teams in a laserinduced CNV mouse model. While Zhang and colleagues reported a decrease of CNV area in mice intravitreally injected with miR-155 mimic [126], Zhuang and colleagues reported the same phenotype but with miR-155 inhibitor [127]. These conflicting findings may result in the different timing used for CNV area calculation as the first team measured CNV area 7 days after laser induction while the other measured 14 days post induction. Surprisingly, downregulation of an endothelial-cell specific microRNA, miR-126 [128], was observed during CNV progression and subsequent intravitreal injection of miR-126 mimic successfully decreased vascular phenotype [129]. Finally, a functional role in CNV progression was also determined for the well-studied let-7 microRNA family [130]. Let-7 transgenic mice exhibit exacerbated expression of let-7a, $\mathrm{d}$ and $\mathrm{f}$ members and were less 
prone to CNV compared to WT mice under laser induction. Concomitantly, subretinal delivery of let-7 inhibitor increased CNV progression in WT mice.

Taken together, these different studies of microRNA implication in AMD strongly support the hypothesis of a functional regulation of AMD by short non-coding RNAs. However, many inconsistencies exist between studies, even when methodological procedures only slightly differ. Furthermore, opposite functions have also been demonstrated for a single microRNA during $\mathrm{CNV}$ progression, drawing a veil on its true biological function in a CNV context. Only extensive combination of new human and animal model studies will allow to tightly decipher microRNA regulation in AMD, and thus eventually be the starting material for new performant drug design. 


\section{- Diabetic retinopathy and microRNAs}

\section{Epidemiology, etiology and animal models}

Diabetes Mellitus (DM) is one of the fastest growing chronic disease worldwide, affecting 463 million individuals in 2019 and excepted to affect 693 million by 2045 [131, 132]. Diabetes can lead to severe complications in many organs, including the eye. Thus, diabetic retinopathy (DR) is a major complication of diabetes, affecting the retina and possibly leading to vision impairment and vision loss. DR affects $35,4 \%$ of all-type diabetic patients and is the fifth leading cause of blindness worldwide [133]. While worldwide DR prevalence in type-1 DM patients is estimated to range from 10 to $50 \%$, with great variations between countries, it is estimated that about $25 \%$ of type- 2 DM patients suffer from DR. DR is a neurovascular pathology triggered by hyperglycemia, affecting different cell-type of the retina and involving several biochemical modifications [134]. It can be classified as nonproliferative diabetic retinopathy (NPDR), proliferative diabetic retinopathy (PDR) and diabetic macular edema (DME). While NPDR is not sight-threatening, PDR form is characterized by retinal neovascularization, a process leading to severe vision impairment. The risk factors for developing DR are glycated hemoglobin, hypertension, dyslipidemia, diabetes duration, ethnic origin, pregnancy, puberty and cataract surgery [135]. At the experimental level, different animal models of DR exist such as the streptozotocin (STZ)induced rodent models, the $d b / d b$ mouse, and the Akimba mouse showing severe retinal neovascularization. All above mentioned models showing their own limitations [136, 137]. Thus, there is still a need for new animal models reflecting every stages of DR progression.

\section{Pathophysiology}

DR is a highly complex neurovascular disease with a not fully understood pathophysiology. As for AMD, the two main biological processes driving DR are angiogenesis and inflammation and are not independent, but rather interacting processes which share several mediators and signaling pathways [138-140] (Figure 7).

Hyperglycemia is responsible for the initiation of molecular and cellular complications, ultimately leading to sigh-threatening endpoints, i.e. PDR and DME. By triggering several biochemical abnormalities, hyperglycemia induces the overproduction of mitochondrial superoxide in vascular endothelial cells and leads to increased flux through the polyol pathway, overactivity of the hexosamine pathway, activation of the protein kinase $\mathrm{C}$ (PKC) and the accumulation of AGEs [141]. These pathways increase the levels of intracellular reactive oxygen species (ROS), and thus oxidative stress, inducing inflammation, vascular hyperpermeability, neuroglial dysfunction and microvascular dysfunction. The levels of proinflammatory cytokines and chemokines such as TNF-alpha, IL-beta, IL6, iNOS and ICAM1 are elevated in DR eyes [142]. Thus, inflammation has pivotal functions in the initiation and progression of DR. In diabetic retinas, leukostasis might damage endothelial cells and neuroglial cells by physical occlusion of capillaries and through the release of inflammatory mediators [143]. This results in a loss of endothelial cells (ECs) and pericytes, subsequently leading to microaneurysms, acellular capillaries and hypoperfusion. Besides the 
overexpression of pro-inflammatory cytokines, VEGF expression is also upregulated in DR, triggered by local ischemia. Diabetic patients with either PDR or DME have elevated vitreous level of VEGF [144]. Elevated levels of VEGF induce neovascularization as well as BRB breakdown, subsequently leading to either PDR or DME.

Among other cellular processes, initial inflammation triggering results in a subsequent deleterious microglia activation in the diabetic retina, leading to neurotoxicity and tissue damage [145].

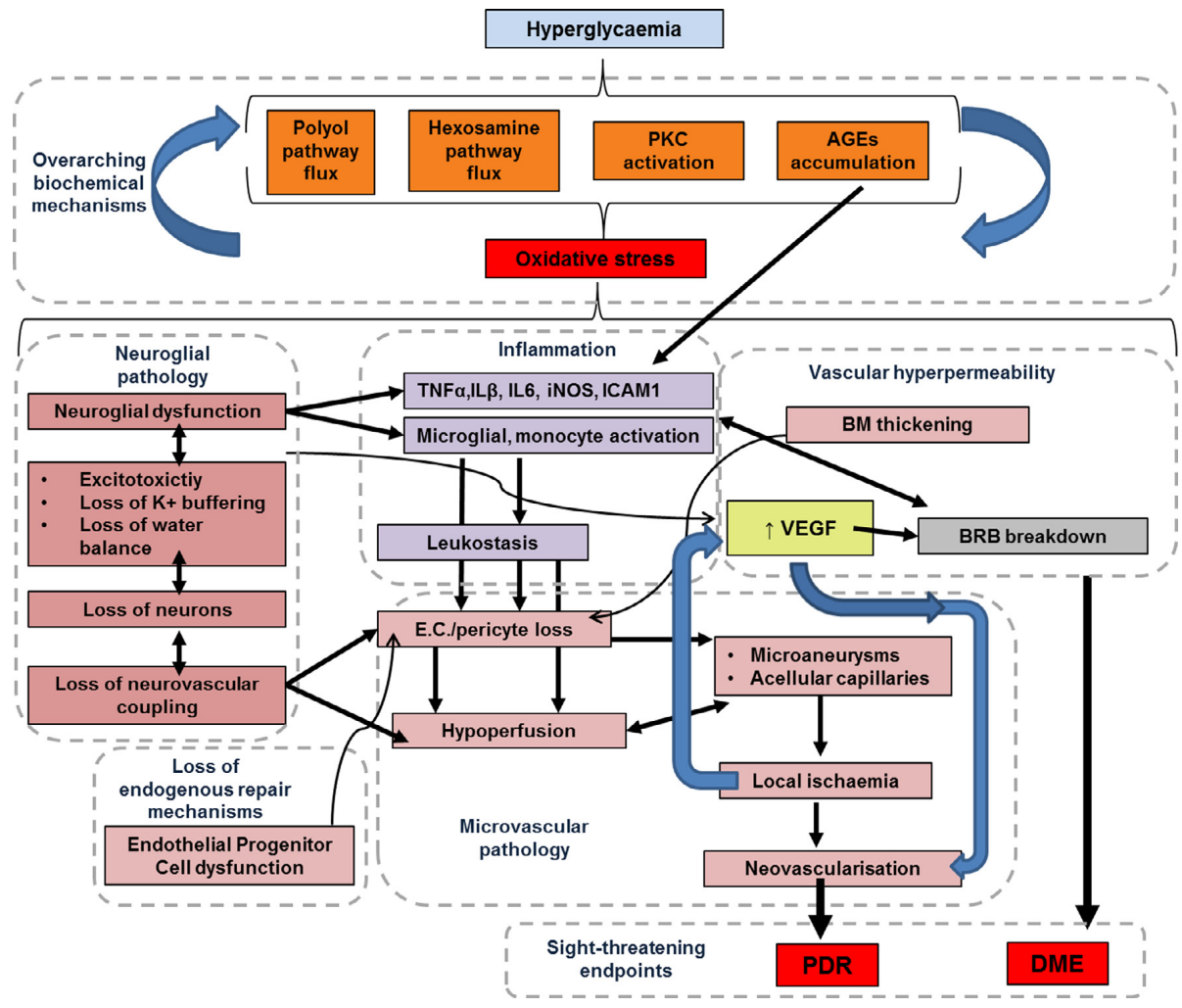

\section{Figure 7. Pathophysiology of diabetic retinopathy}

Diabetic retinopathy is triggered by hyperglycemia, which induces diverse molecular and cellular complications ultimately leading to sigh-threatening endpoints. Polyol pathway, hexosamine pathway, PKC activation and AGEs accumulation all lead to inflammation, vascular hyperpermeability, neuroglial pathology and microvascular pathology, finally resulting in BRB breakdown and neovascularization. Neuroglial pathology is mainly characterized by loss of neuros and neurovascular coupling while early microvascular pathology results in EC and pericyte loss, overall resulting in neovascularization. Several inflammatory and angiogenic mediators are involved, such as but not limited to TNF $\alpha$, IL1- $\beta$, IL6, iNOS and VEGF. Final endpoints triggered by hyperglycemia are PDR and DME.

Adapted from Lechner et al. (2017) 


\section{Clinical management}

As DR incidence in diabetic patients increases with age, DR onset has to be strictly monitored through regular eye examination. Thus, it is recommended that diabetic patients showing no signs of DR undergo ophthalmology follow-up every 1-2 year to attest any DR development [134]. While mild and moderate NPDR are generally not treated, severe NPDR as well as PDR et DME can be partly treated. For severe NPDR, panretinal photocoagulation is considered for ablating ischemic neurons and glial cells in non-perfusion areas [146, 147]. However, despite panretinal photocoagulation can reduce the risk of developing PDR, its intrinsic destructive properties can cause peripheral visual field defects, reduced night vision and eventually exacerbates DME [148]. For PDR, panretinal photocoagulation can also applied to avoid vitreous hemorrhages, retinal detachment and eventually glaucoma [146]. Sustained vitreous hemorrhage or retinal detachment can be overcome by vitrectomy. Notably, the emergence of anti-VEGF therapy first designed for AMD treatment had also considerably improved DR clinical management. Thus, anti-VEGF therapy is now a standard procedure for DME and PDR treatment [149]. While bevacizumab has been used has an offlabel drug for DME management since its approval by FDA for colorectal cancer in 2004, ranibizumab and aflibercept were approved by the FDA for DME treatment in 2012 and 2014, respectively [150]. Since August 2020, the BUZZARD study is currently addressing the safety and efficacy of brolucizumab in the treatment of DME (clinicaltrial.gov). Another alternative for the management of DME, notably for DME patient refractory to anti-VEGF, is the use of corticosteroids [149]. Several candidates are currently being tested or in development for DME management such as angiopoietin pathway stimulators and Rhoassociated protein kinase inhibitors [151].

\section{MicroRNA implication}

As for AMD, the next big step in DR treatment could arose from microRNA studies, but to achieve this goal, an in-depth understanding of microRNA regulation of DR is mandatory. Several studies have currently focused on establishing microRNAs has potential biomarkers or therapeutic targets in AMD in both human and mouse study.

Because vitrectomy surgery can be performed to overcome vitreous hemorrhage and retinal detachment in DR patients, vitreous fluid has been broadly used as a biological fluid for miRNA detection in human. Serum, plasma, blood extracellular vesicles, peripheral blood and endothelial progenitor cells have also been studied for a microRNA signature in DR patients. Li and Martinez recently reviewed the main findings of such studies [152, 153]. In rodent, many studies have focused on microRNA profiling in STZ-induced rat, mainly in either retinas or isolated retinal endothelial cells (RECs) [154]. Streptozotocin (STZ) is a cytotoxic agent leading to $\beta$-pancreatic cell death, possibly leading thus to diabetes. Its administration to rodents (mouse or rat) is broadly used to trigger DR phenotype [155]. Kovacs and colleagues performed a microarray analysis on retinas and RECs from STZinduced rats 3 months after diabetes onset and identified a specific set of dysregulated microRNAs [156]. Surprisingly, retinas and RECs microRNAs profiles exhibited large variations, probably due to a more complex cellular population in retinas. In a similar study 
conducted by $\mathrm{Wu}$ and colleagues, microRNA expression profiles in retinas of STZ-rat was conducted during DR progression until 10 weeks after STZ injection [157]. Interestingly, relative levels of miR-182, 96, 183, 211, 204 and 124 were positively correlated with DR progression while relative levels of miR-10b, 10a, 219-2-3p, 144, 338 and 199a-3p negatively correlated. Unfortunately, Kovacs and $\mathrm{Wu}$ profiling showed almost no overlap in microRNA dysregulation. Finally, Gong and colleagues profiled microRNA dysregulation in either human RECs or human RPE under hyperglycemic conditions and analyzed the same set of microRNAs in the retinas of STZ-rat over time [158]. In these studies, no functional analysis of specifically dysregulated microRNAs was made. Other studies aimed to determine a potential functional role of specific microRNA in different DR models. Among others, it has been thus demonstrated that miR-29a/b, miR-146a, miR-199a-3p and miR-200b alter DR onset in either in vitro or in vivo models [159-162]. Of particular interest is the opposite function of let-7 in NPDR and wet AMD [130]. While silencing of let-7 microRNA in the eye increased laser-induced CNV, transgenic mice overexpressing let-7 family exhibit characteristics of NPDR, suggesting distinct implication in the pathogenesis of DR and AMD.

All together, these different studies on microRNA implication in DR strongly support the hypothesis of a functional regulation in the course of this pathology. However, many inconsistencies exist between studies, even when methodological procedures only slightly differ. Only extensive combination of new human and animal model studies will allow to tightly decipher microRNA regulation in DR, and thus eventually be the starting material for new performant drug design.

\section{- MicroRNA in other eye conditions}

Although microRNA implication in AMD and DR is extensively studied in the scientific literature, microRNA implication in many other eye conditions is also described. Thus, microRNAs as potential biomarkers or therapeutic tools for glaucoma, retinitis pigmentosa, cataract, keratitis and uveitis have been described [163-167]. 


\section{MicroRNA in retinal microglia}

Glial cells are non-neuronal cells maintaining homeostasis of the central nervous system (CNS) and peripheral nervous system, producing myelin and providing support and protection for neurons. Glial cells are a cell type population composed of microglia and macroglia, which includes astrocytes, oligodendrocytes, ependymal cells and retina-specific Mullër cells. Microglia are the resident macrophages of the central nervous system (CNS), including the retina, and play pivotal functions in developmental, physiological and pathological events $[168,169]$.

A
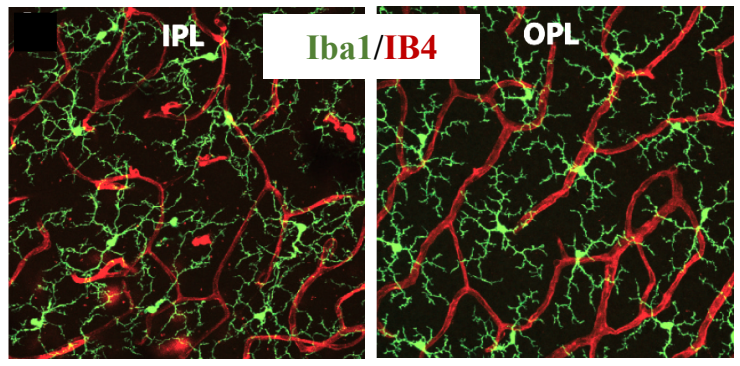

B
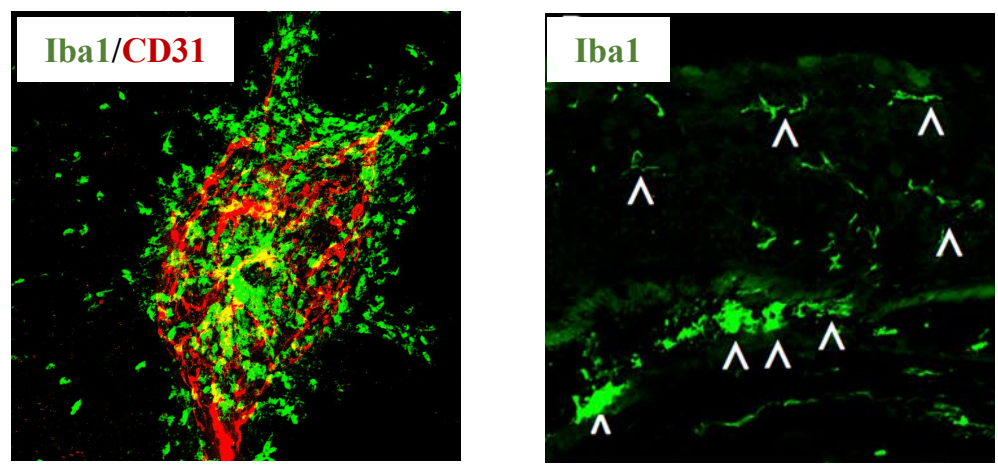

Figure 8. Retina microglia cells, sentinels of the eye

Microglia cells are the resident macrophages of the retina and are involved in both physiological and pathological conditions.

A. In the healthy retina, microglia (Iba1 stained, in green) form a stable and highly ordered network of ramified cells, closely linked to the vascular network (IB4 stained, in red), with their cell bodies located both at the inner plexiform layer (IPL) and outer plexiform layer (OPL). Flatmounted retina, adapted from Silverman and Wong (2018).

B. Microglial cells are major actors of many retinal disease such as AMD and DR.

Left panel: flat-mounted choroid stained for microglia cells (in green) and vascular network (in red) in the CNV lesion region of the laser-induced CNV mouse model.

Right panel: transversal retinal section stained for microglia cells (in green, shown by white arrowheads) in the Akimba mouse model. Adapted from Chaurasia et al. (2018). 
During development, microglia derive from primitive hematopoietic progenitors originating from the embryonic yolk sac and colonize the mouse brain and retina at E9 and E11.5, respectively $[170,171]$. Retinal microglia colonize the retina in a radial and vitrealto-scleral direction, starting near the optic nerve. During colonization, microglia cells proliferate, maturate and gain their final branched morphology (i.e. small cell bodies with long cellular protrusions). In the course of development, microglia are responsible of the survival and death of developing retinal neurons, the shaping of neuronal circuits and the modeling of the retinal vasculature [172-174]. During adult life, microglia cells form a stable and highly ordered network of ramified cells, with their stroma located at the inner plexiform layer (IPL) and outer plexiform layer (OPL), and carry out constitutive maintenance functions in order to maintain tissue integrity [171] (Figure 8). Throughout adulthood, microglia constantly screen their local environment with their long protrusions for eventual pathogen invasion and assure neuronal activity and synaptic integrity [175]. Recent findings suggest that adult retina microglia cells exist as a self-sustaining, closed population in the absence of injury or disease, being thus able of mild proliferation [176, 177]. Retina microglia cells remain quiescent and insure homeostasis under the control of both RPE cells and neurons [178, 179], but can shift at any moment into an activated mode under pathological conditions.

Classical molecular markers used for the study of general microglia include CD68, CD11b, F4/80, CX3CR1 and Iba1 [168]. Unfortunately, these markers can sometimes also be expressed by peripheral macrophages and should be used with care as discrimination can be tricky (Figure 9A). In a recent work, Jurga and colleagues review the latest findings on specific general microglia markers as well as on specific activation microglia marker [180]. Thus, combining the use of classical marker (e.g. Iba1) and the use of expression profile of other markers (e.g. CD45 ${ }^{\text {low }}$ ) may be a suitable strategy for specific microglia study.

Microglia activation may be analyzed by both morphological parameters and molecular markers. This activation can be triggered by several mediators such as but not limited to lipopolysaccharides (LPS), tumor necrosis alpha (TNF- $\alpha$ ), interferon gamma (IFN- $\gamma$ ), diverse complement factors, extracellular ATP, amyloid- $\beta$, TGF- $\beta$, IL-4 and IL-10 [180, 181] (Figure 9B). While morphological analysis will allow to characterize activated state versus quiescent state, molecular markers analysis will allow to discriminate M1 activated phenotype (pro-inflammatory) versus M2 activated phenotype (anti-inflammatory). Activation of microglia cells is thus morphologically observed as a change from a highly ramified shape to an amoeboid shape, showing thus a direct form-to-function relationship. However, this morphological change does not result in a binary state between quiescent and activated microglia, but rather in a wide range of intermediate morphologies, the traditional view of a "ramified resting" and "amoeboid activated" being overcome [182]. Different shape parameters have been described to study this morphological shift [183]. At the molecular level, activation of microglia cells can be observed through the production of different intracellular, surface and secretory markers (Figure 9B). Thus, M1 activated microglia cell will exhibit high intracellular level of TSPO (Translocator protein) and iNOS 
as long with the production of CD14, CD16, CD32, CD40, CD86 and different MHCII proteins. These M1 microglia will also secrete different cytokines and chemokines such as IL-6 and CXCL1.

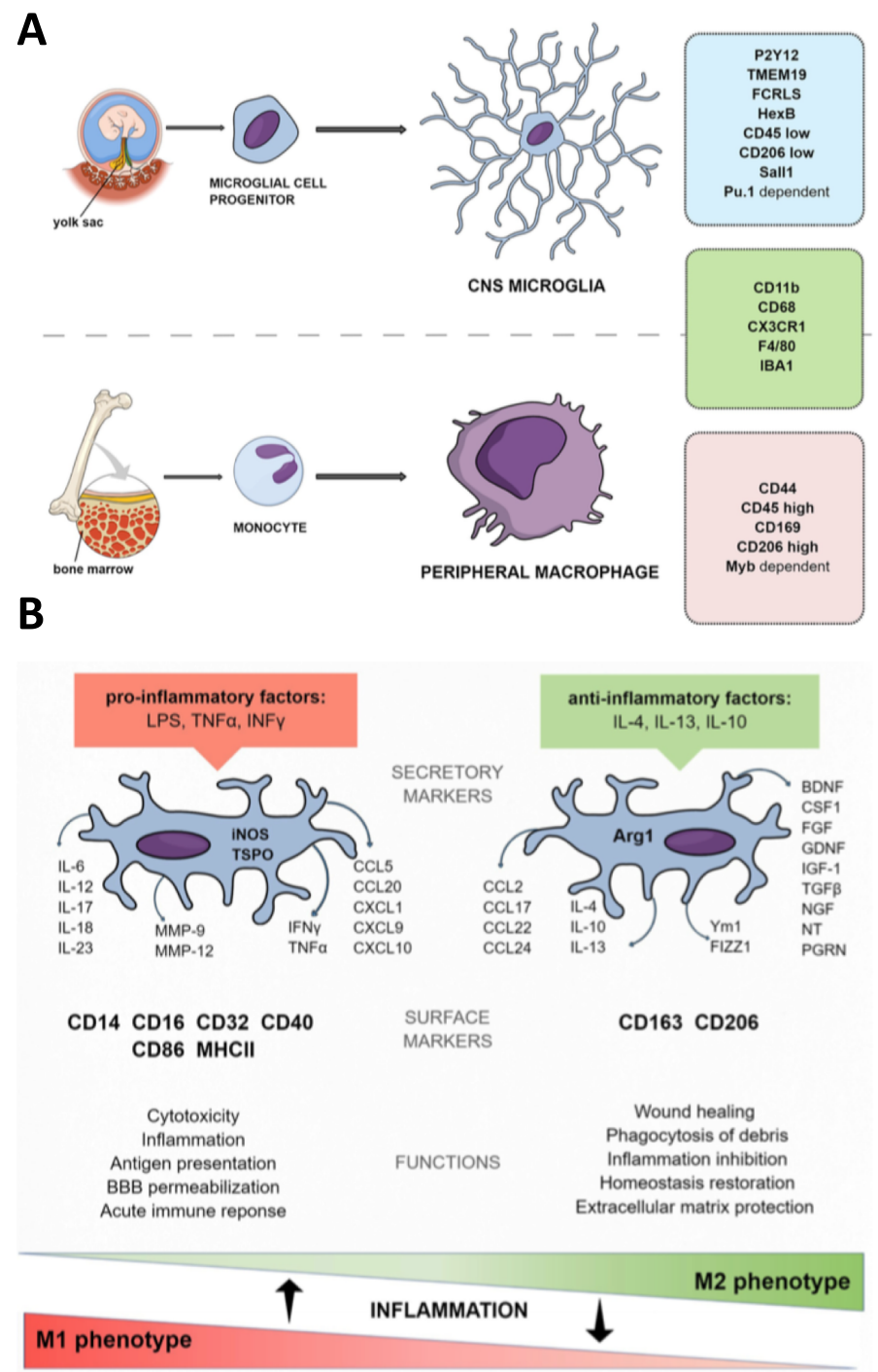

Figure 9. Markers of general and activated microglia

A. Microglia and peripheral macrophages share different molecular markers, but also have distinct expression patterns. Thus, microglia cells, but not peripheral macrophages, are positive for P2Y12, TMEM19, FCRL5, HexB and show a low basal level of CD45.

B. Activated microglia cells may exhibit a M1 (pro-inflammatory) or M2 (anti-inflammatory) phenotype, or any intermediate phenotype. Distinction between Ma and M2 phenotype is possible through the analysis of different secretory, surface or intracellular markers.

Adapted from Jurga (2020). 
Because of their activation in a broad range of neurovascular complications including DR and AMD, microglia as therapeutic targets could limit inflammatory changes and thus successfully alleviate disease progression. Thus, several inflammatory mediators linked to microglia activation are currently used or under investigations for clinical application. Synthetic glucocorticoids can bind to endogenous glucocorticoid receptor and inhibit activation of the innate immune system through the negative regulation of pro-inflammatory molecular pathways [184]. In the clinic, delivery of glucocorticoids via intravitreal injections or sustained-release implant have been used for the treatment of DME and retinal vein occlusion $[185,186]$. Unfortunately, the direct contribution of microglia modulation to the overall effects of the therapy remains elusive. Minocycline, a tetracycline derivative, is a highly bioavailable agent decreasing microglia activation via mechanisms involving the inhibition of MAPK [187]. After showing promising therapeutic effects in multiple retinal disease models [188, 189], it has entered clinical trial for the treatment of DME, showing a modest but progressive improvement of disease [190].

MicroRNAs and transcription factors work together to orchestrate complex cellular events, such as monocyte-macrophage differentiation as well as microglia development and activation. CEBP $\alpha$ and PU.1 are two transcription factors known for their critical regulation of monocyte to macrophage differentiation and microglia development, CEBP being considered the master regulator of hematopoietic stem cell differentiation. On one hand, CEBP $\alpha$ transcription factor directly regulates several microRNAs, including miR-223 by activating miR-223 gene transcription [191]. On the other hand, PU.1 is required to promote the skewing of granulocyte-macrophage progenitors to the monocyte lineage. High level of PU.1 induce the transcription of miR-424 gene and thus avoid favoring the granulocyte lineage, with combinatorial regulation of miR-222 and miR-155 [192]. In addition, the miR17-92 cluster has been directly associated with the process of monocyte to macrophage differentiation [193]. Given the large similarities between macrophages and microglia, one can hypothesize that some of these microRNAs and transcription factors also play critical role in microglia differentiation. Some studies focused on the specific role of microRNA in the development of microglia, rather than the monocyte to macrophage differentiation. Thus, miR-124, one of the most abundant microRNAs in the brain, is required for the proper maintenance of the quiescent state of microglia in the brain, by targeting CEBP $\alpha$ as well as CDK4 and CDK6, two cyclins [194, 195]. MiR-155, a broadly described pro-inflammatory microRNA, targets anti-inflammatory proteins in microglia such as SOCS1, leading to the upregulation of several inflammatory mediators and contributing to a microglia-mediated neurotoxic response [196]. Several other microRNAs, such as miR-181a-5p, miR-30a and miR-26b-5p, have been directly linked to brain or retina microglia activation, highlighting a critical regulation on microglia physiology [197-199]. Of particular interest, miR-142-3p has been recently linked to microglia activation in rat by Gupta and colleagues [200]. The authors performed a microRNA microarray in LPS activated microglia derived from rat pup's brain and identified miR-142-3p as the top up-regulated microRNA. In this context, they identified CAMK2A $\left(\mathrm{Ca}^{2+} /\right.$ calmodulin dependent kinase 2a) as a direct target of miR-142, a gene 
implicated in the regulation of the expression of brain-derived neurotrophic factor and longterm potentiation, a cellular mechanism underlying memory and learning.

All together, these results demonstrate the prominent role of microRNAs in the regulation of microglial development and activation, opening new interesting opportunities for anti-inflammatory therapies. Further studies will have to determine how miRNA modulation could interfere with microglia biology in specific pathological conditions, possibly leading to new promising clinical candidates. 


\section{References}

1. Zeng, J., et al., Cross-Kingdom Small RNAs Among Animals, Plants and Microbes. Cells, 2019. 8(4).

2. Lee, R.C., R.L. Feinbaum, and V. Ambros, The C. elegans heterochronic gene lin4 encodes small RNAs with antisense complementarity to lin-14. Cell, 1993. 75(5): p. 843-54.

3. Kozomara, A., M. Birgaoanu, and S. Griffiths-Jones, miRBase: from microRNA sequences to function. Nucleic Acids Res, 2019. 47(D1): p. D155-d162.

4. Felekkis, K., et al., microRNAs: a newly described class of encoded molecules that play a role in health and disease. Hippokratia, 2010. 14(4): p. 236-40.

5. Bartel, D.P., MicroRNAs: genomics, biogenesis, mechanism, and function. Cell, 2004. 116(2): p. 281-97.

6. $\quad$ Dragomir, M., et al., Using microRNA Networks to Understand Cancer. Int J Mol Sci, 2018. 19(7).

7. Wojciechowska, A., A. Braniewska, and K. Kozar-Kaminska, MicroRNA in cardiovascular biology and disease. Adv Clin Exp Med, 2017. 26(5): p. 865-874.

8. $\quad \mathrm{Xu}, \mathrm{S}$. and L.D. Hazlett, MicroRNAs in Ocular Infection. Microorganisms, 2019. 7(9).

9. Raghunath, A. and E. Perumal, Micro-RNAs and their roles in eye disorders. Ophthalmic Res, 2015. 53(4): p. 169-86.

10. Bartel, D.P., Metazoan MicroRNAs. Cell, 2018. 173(1): p. 20-51.

11. O'Brien, J., et al., Overview of MicroRNA Biogenesis, Mechanisms of Actions, and Circulation. Front Endocrinol (Lausanne), 2018. 9: p. 402.

12. Gantier, M.P., et al., Analysis of microRNA turnover in mammalian cells following Dicerl ablation. Nucleic Acids Res, 2011. 39(13): p. 5692-703.

13. Okamura, K., N. Liu, and E.C. Lai, Distinct mechanisms for microRNA strand selection by Drosophila Argonautes. Mol Cell, 2009. 36(3): p. 431-44.

14. Guo, L., et al., Evolutionary and expression analysis of miR-\#-5p and miR-\#-3p at the miRNAs/isomiRs levels. Biomed Res Int, 2015. 2015: p. 168358.

15. Li, H., et al., MicroRNA-296: a promising target in the pathogenesis of atherosclerosis? Mol Med, 2018. 24(1): p. 12.

16. Hutvágner, G., et al., A cellular function for the RNA-interference enzyme Dicer in the maturation of the let-7 small temporal RNA. Science, 2001. 293(5531): p. 8348.

17. Jones-Rhoades, M.W., D.P. Bartel, and B. Bartel, MicroRNAS and their regulatory roles in plants. Annu Rev Plant Biol, 2006. 57: p. 19-53.

18. Shin, C., et al., Expanding the microRNA targeting code: functional sites with centered pairing. Mol Cell, 2010. 38(6): p. 789-802.

19. Jonas, S. and E. Izaurralde, Towards a molecular understanding of microRNAmediated gene silencing. Nat Rev Genet, 2015. 16(7): p. 421-33.

20. Eichhorn, S.W., et al., $m R N A$ destabilization is the dominant effect of mammalian microRNAs by the time substantial repression ensues. Mol Cell, 2014. 56(1): p. 104-15.

21. Bartel, D.P., MicroRNAs: target recognition and regulatory functions. Cell, 2009. 136(2): p. 215-33.

22. Agarwal, V., et al., Predicting effective microRNA target sites in mammalian $m R N A s$. Elife, 2015. 4. 
23. Riffo-Campos, A.L., I. Riquelme, and P. Brebi-Mieville, Tools for SequenceBased miRNA Target Prediction: What to Choose? Int J Mol Sci, 2016. 17(12).

24. Chou, C.H., et al., miRTarBase update 2018: a resource for experimentally validated microRNA-target interactions. Nucleic Acids Res, 2018. 46(D1): p. D296-d302.

25. Wong, N. and X. Wang, miRDB: an online resource for microRNA target prediction and functional annotations. Nucleic Acids Res, 2015. 43(Database issue): p. D146-52.

26. Baskerville, S. and D.P. Bartel, Microarray profiling of microRNAs reveals frequent coexpression with neighboring miRNAs and host genes. Rna, 2005. 11(3): p. 241-7.

27. Ozsolak, F., et al., Chromatin structure analyses identify miRNA promoters. Genes Dev, 2008. 22(22): p. 3172-83.

28. Ha, M. and V.N. Kim, Regulation of microRNA biogenesis. Nat Rev Mol Cell Biol, 2014. 15(8): p. 509-24.

29. Ghini, F., et al., Endogenous transcripts control miRNA levels and activity in mammalian cells by target-directed miRNA degradation. Nat Commun, 2018. 9(1): p. 3119.

30. Pawlica, P., et al., How Complementary Targets Expose the microRNA 3' End for Tailing and Trimming during Target-Directed microRNA Degradation. Cold Spring Harb Symp Quant Biol, 2019. 84: p. 179-183.

31. Krol, J., et al., Characterizing light-regulated retinal microRNAs reveals rapid turnover as a common property of neuronal microRNAs. Cell, 2010. 141(4): p. 618-31.

32. Marzi, M.J., et al., Degradation dynamics of microRNAs revealed by a novel pulse-chase approach. Genome Res, 2016. 26(4): p. 554-65.

33. Seki, N. and A. Hata, Noncoding RNAs: a new fine-tuner is a key player of human pathogenesis. J Hum Genet, 2017. 62(1): p. 1.

34. Selbach, M., et al., Widespread changes in protein synthesis induced by microRNAs. Nature, 2008. 455(7209): p. 58-63.

35. Wurdinger, T. and F.F. Costa, Molecular therapy in the microRNA era. Pharmacogenomics J, 2007. 7(5): p. 297-304.

36. Fuziwara, C.S. and E.T. Kimura, Insights into Regulation of the miR-17-92 Cluster of miRNAs in Cancer. Front Med (Lausanne), 2015. 2: p. 64.

37. Witwer, K.W. and M.K. Halushka, Toward the promise of microRNAs Enhancing reproducibility and rigor in microRNA research. RNA Biol, 2016. 13(11): p. 1103-1116.

38. Jones, D., Setbacks shadow microRNA therapies in the clinic. Nat Biotechnol, 2018. 36(10): p. 909-910.

39. Hanna, J., G.S. Hossain, and J. Kocerha, The Potential for microRNA Therapeutics and Clinical Research. Front Genet, 2019. 10: p. 478.

40. Stenvang, J., et al., Inhibition of microRNA function by antimiR oligonucleotides. Silence, 2012. 3(1): p. 1.

41. Dang, K. and K.A. Myers, The role of hypoxia-induced miR-210 in cancer progression. Int J Mol Sci, 2015. 16(3): p. 6353-72.

42. Fan, J., et al., A simplified system for the effective expression and delivery of functional mature microRNAs in mammalian cells. Cancer Gene Ther, 2020. 27(6): p. 424-437. 
43. Shrestha, A., et al., MicroRNA-142 is a multifaceted regulator in organogenesis, homeostasis, and disease. Dev Dyn, 2017. 246(4): p. 285-290.

44. Chen, C.Z., et al., MicroRNAs modulate hematopoietic lineage differentiation. Science, 2004. 303(5654): p. 83-6.

45. Nishiyama, T., et al., miR-142-3p is essential for hematopoiesis and affects cardiac cell fate in zebrafish. Biochem Biophys Res Commun, 2012. 425(4): p. 755-61.

46. Lu, X., et al., miR-142-3p regulates the formation and differentiation of hematopoietic stem cells in vertebrates. Cell Res, 2013. 23(12): p. 1356-68.

47. Chapnik, E., et al., miR-142 orchestrates a network of actin cytoskeleton regulators during megakaryopoiesis. Elife, 2014. 3: p. e01964.

48. Shrestha, A., et al., Generation and Validation of miR-142 Knock Out Mice. PLoS One, 2015. 10(9): p. e0136913.

49. Sladitschek, H.L. and P.A. Neveu, The bimodally expressed microRNA miR-142 gates exit from pluripotency. Mol Syst Biol, 2015. 11(12): p. 850.

50. Carraro, G., et al., miR-142-3p balances proliferation and differentiation of mesenchymal cells during lung development. Development, 2014. 141(6): p. 127281.

51. Sharma, S., et al., Repression of miR-142 by p300 and MAPK is required for survival signalling via gp130 during adaptive hypertrophy. EMBO Mol Med, 2012. 4(7): p. 617-32.

52. Danger, R., et al., Upregulation of miR-142-3p in peripheral blood mononuclear cells of operationally tolerant patients with a renal transplant. J Am Soc Nephrol, 2012. 23(4): p. 597-606.

53. Deng, B., et al., MicroRNA-142-3p inhibits cell proliferation and invasion of cervical cancer cells by targeting FZD7. Tumour Biol, 2015. 36(10): p. 8065-73.

54. Wu, L., et al., MicroRNA-142-3p, a new regulator of RAC1, suppresses the migration and invasion of hepatocellular carcinoma cells. FEBS Lett, 2011. 585(9): p. 1322-30.

55. Ma, Z., et al., MicroRNA regulatory pathway analysis identifies miR-142-5p as a negative regulator of TGF- $\beta$ pathway via targeting SMAD3. Oncotarget, 2016. 7(44): p. 71504-71513.

56. Mansoori, B., et al., miR-142-3p is a tumor suppressor that inhibits estrogen receptor expression in ER-positive breast cancer. J Cell Physiol, 2019.

57. Mandolesi, G., et al., miR-142-3p Is a Key Regulator of IL-1beta-Dependent Synaptopathy in Neuroinflammation. J Neurosci, 2017. 37(3): p. 546-561.

58. Zhang, T., C. Ji, and R. Shi, miR-142-3p promotes pancreatic $\beta$ cell survival through targeting FOXO1 in gestational diabetes mellitus. Int J Clin Exp Pathol, 2019. 12(5): p. 1529-1538.

59. Peng, D., et al., miR-142-3p suppresses uveal melanoma by targeting CDC25C, TGFbetaR1, GNAQ, WASL, and RAC1. Cancer Manag Res, 2019. 11: p. 47294742.

60. Huang, P., et al., MicroRNA Expression Patterns Involved in Amyloid BetaInduced Retinal Degeneration. Invest Ophthalmol Vis Sci, 2017. 58(3): p. 17261735.

61. Gehring, W.J., New perspectives on eye development and the evolution of eyes and photoreceptors. J Hered, 2005. 96(3): p. 171-84. 
62. Lamb, T.D., S.P. Collin, and E.N. Pugh, Jr., Evolution of the vertebrate eye: opsins, photoreceptors, retina and eye cup. Nat Rev Neurosci, 2007. 8(12): p. 960-76.

63. Nakao, S., A. Hafezi-Moghadam, and T. Ishibashi, Lymphatics and lymphangiogenesis in the eye. J Ophthalmol, 2012. 2012: p. 783163.

64. Parchem, R.J., et al., miR-302 Is Required for Timing of Neural Differentiation, Neural Tube Closure, and Embryonic Viability. Cell Rep, 2015. 12(5): p. 760-73.

65. Dambal, S., et al., The microRNA-183 cluster: the family that plays together stays together. Nucleic Acids Res, 2015. 43(15): p. 7173-88.

66. Lumayag, S., et al., Inactivation of the microRNA-183/96/182 cluster results in syndromic retinal degeneration. Proc Natl Acad Sci U S A, 2013. 110(6): p. E507-16.

67. Xiang, L., et al., miR-183/96 plays a pivotal regulatory role in mouse photoreceptor maturation and maintenance. Proc Natl Acad Sci U S A, 2017. 114(24): p. 6376-6381.

68. Barbato, S., et al., MiR-211 is essential for adult cone photoreceptor maintenance and visual function. Sci Rep, 2017. 7(1): p. 17004.

69. Sanuki, R., et al., miR-124a is required for hippocampal axogenesis and retinal cone survival through Lhx2 suppression. Nat Neurosci, 2011. 14(9): p. 1125-34.

70. Sundermeier, T.R. and K. Palczewski, The impact of microRNA gene regulation on the survival and function of mature cell types in the eye. Faseb j, 2016. 30(1): p. 23-33.

71. Soundara Pandi, S.P., et al., Extremely complex populations of small RNAs in the mouse retina and RPE/choroid. Invest Ophthalmol Vis Sci, 2013. 54(13): p. 814051.

72. Karali, M., et al., High-resolution analysis of the human retina miRNome reveals isomiR variations and novel microRNAs. Nucleic Acids Res, 2016. 44(4): p. 152540 .

73. Karali, M., et al., miRNeye: a microRNA expression atlas of the mouse eye. BMC Genomics, 2010. 11: p. 715.

74. Ragusa, M., et al., MicroRNAs in vitreus humor from patients with ocular diseases. Mol Vis, 2013. 19: p. 430-40.

75. Flaxman, S.R., et al., Global causes of blindness and distance vision impairment 1990-2020: a systematic review and meta-analysis. The Lancet Global Health, 2017. 5(12): p. e1221-e1234.

76. Wong, W.L., et al., Global prevalence of age-related macular degeneration and disease burden projection for 2020 and 2040: a systematic review and metaanalysis. The Lancet Global Health, 2014. 2(2): p. e106-e116.

77. Adelman, R.A., E.J. Sigler, and R.K. Seth, Age-related Macular DegenerationReview and Current Concepts. US Ophthalmic Review, 2011. 04(01).

78. Lambert, N.G., et al., Risk factors and biomarkers of age-related macular degeneration. Prog Retin Eye Res, 2016. 54: p. 64-102.

79. Grossniklaus, H.E. and W.R. Green, Choroidal neovascularization. Am J Ophthalmol, 2004. 137(3): p. 496-503.

80. Campochiaro, P.A., Ocular neovascularization. J Mol Med (Berl), 2013. 91(3): p. 311-21.

81. Pennesi, M.E., M. Neuringer, and R.J. Courtney, Animal models of age related macular degeneration. Mol Aspects Med, 2012. 33(4): p. 487-509. 
82. Lambert, V., et al., Laser-induced choroidal neovascularization model to study age-related macular degeneration in mice. Nat Protoc, 2013. 8(11): p. 2197-211.

83. Kwak, N., et al., VEGF is major stimulator in model of choroidal neovascularization. Invest Ophthalmol Vis Sci, 2000. 41(10): p. 3158-64.

84. Rakic, J.M., et al., Placental growth factor, a member of the VEGF family, contributes to the development of choroidal neovascularization. Invest Ophthalmol Vis Sci, 2003. 44(7): p. 3186-93.

85. Lambert, N.G., et al., Subretinal AAV2.COMP-Ang1 suppresses choroidal neovascularization and vascular endothelial growth factor in a murine model of age-related macular degeneration. Exp Eye Res, 2016. 145: p. 248-257.

86. Iwase, T., et al., Sustained delivery of a HIF-1 antagonist for ocular neovascularization. J Control Release, 2013. 172(3): p. 625-33.

87. Bhutto, I.A., et al., Pigment epithelium-derived factor (PEDF) and vascular endothelial growth factor (VEGF) in aged human choroid and eyes with agerelated macular degeneration. Exp Eye Res, 2006. 82(1): p. 99-110.

88. Wang, S., C.M. Sorenson, and N. Sheibani, Lack of thrombospondin 1 and exacerbation of choroidal neovascularization. Arch Ophthalmol, 2012. 130(5): p. 615-20.

89. Lambert, V., et al., MMP-2 and MMP-9 synergize in promoting choroidal neovascularization. Faseb j, 2003. 17(15): p. 2290-2.

90. Lecomte, J., et al., Bone marrow-derived mesenchymal cells and MMP13 contribute to experimental choroidal neovascularization. Cell Mol Life Sci, 2011. 68(4): p. 677-86.

91. Jost, M., et al., Tumoral and choroidal vascularization: differential cellular mechanisms involving plasminogen activator inhibitor type I. Am J Pathol, 2007. 171(4): p. 1369-80.

92. Parmeggiani, F., et al., Mechanism of inflammation in age-related macular degeneration. Mediators Inflamm, 2012. 2012: p. 546786.

93. Streilein, J.W., et al., Ocular immune privilege and the impact of intraocular inflammation. DNA Cell Biol, 2002. 21(5-6): p. 453-9.

94. Zhou, R., et al., A new look at immune privilege of the eye: dual role for the vision-related molecule retinoic acid. J Immunol, 2011. 187(8): p. 4170-7.

95. Medzhitov, R., Origin and physiological roles of inflammation. Nature, 2008. 454(7203): p. 428-35.

96. Xu, H., M. Chen, and J.V. Forrester, Para-inflammation in the aging retina. Prog Retin Eye Res, 2009. 28(5): p. 348-68.

97. Bhutto, I. and G. Lutty, Understanding age-related macular degeneration (AMD): relationships between the photoreceptor/retinal pigment epithelium/Bruch's membrane/choriocapillaris complex. Mol Aspects Med, 2012. 33(4): p. 295-317.

98. Anderson, D.H., et al., The pivotal role of the complement system in aging and age-related macular degeneration: hypothesis re-visited. Prog Retin Eye Res, 2010. 29(2): p. 95-112.

99. Klein, R.J., et al., Complement factor H polymorphism in age-related macular degeneration. Science, 2005. 308(5720): p. 385-9.

100. Sun, C., M. Zhao, and X. Li, CFB/C2 gene polymorphisms and risk of age-related macular degeneration: a systematic review and meta-analysis. Curr Eye Res, 2012. 37(4): p. 259-71.

101. Tayanithi, P., P. Pisankosakul, and P. Laksakapuk, Treatment of subfoveal choroidal neovascularization secondary to age related macular degeneration with 
single treatment of verteporfin photodynamic therapy: a safety and short-term outcome. J Med Assoc Thai, 2004. 87 Suppl 2: p. S78-82.

102. Maberley, D., Pegaptanib for neovascular age-related macular degeneration. Issues Emerg Health Technol, 2005(76): p. 1-4.

103. Chakravarthy, U., et al., Year 2 efficacy results of 2 randomized controlled clinical trials of pegaptanib for neovascular age-related macular degeneration. Ophthalmology, 2006. 113(9): p. 1508.e1-25.

104. El-Mollayess, G.M., B.N. Noureddine, and Z.F. Bashshur, Bevacizumab and neovascular age related macular degeneration: pathogenesis and treatment. Semin Ophthalmol, 2011. 26(3): p. 69-76.

105. Brown, D.M., et al., Ranibizumab versus verteporfin for neovascular age-related macular degeneration. N Engl J Med, 2006. 355(14): p. 1432-44.

106. Garcia-Layana, A., et al., Treatment of Exudative Age-related Macular Degeneration: Focus on Aflibercept. Drugs Aging, 2015. 32(10): p. 797-807.

107. Nguyen, Q.D., et al., Brolucizumab: Evolution through Preclinical and Clinical Studies and the Implications for the Management of Neovascular Age-Related Macular Degeneration. Ophthalmology, 2020. 127(7): p. 963-976.

108. Yannuzzi, N.A. and K.B. Freund, Brolucizumab: evidence to date in the treatment of neovascular age-related macular degeneration. Clin Ophthalmol, 2019. 13: p. 1323-1329.

109. Zuber-Laskawiec, K., et al., Non-responsiveness and tachyphylaxis to antivascular endothelial growth factor treatment in naive patients with exudative agerelated macular degeneration. J Physiol Pharmacol, 2019. 70(5).

110. Bandello, F., et al., Recent advances in the management of dry age-related macular degeneration: A review. F1000Res, 2017. 6: p. 245.

111. Grassmann, F., et al., A circulating microrna profile is associated with late-stage neovascular age-related macular degeneration. PLoS One, 2014. 9(9): p. e107461.

112. Ren, C., et al., Circulating miRNAs as Potential Biomarkers of Age-Related Macular Degeneration. Cell Physiol Biochem, 2017. 41(4): p. 1413-1423.

113. Ertekin, S., et al., Evaluation of circulating miRNAs in wet age-related macular degeneration. Mol Vis, 2014. 20: p. 1057-66.

114. Ménard, C., et al., MicroRNA signatures in vitreous humour and plasma of patients with exudative AMD. Oncotarget, 2016. 7(15): p. 19171-84.

115. Romano, G.L., et al., Retinal and Circulating miRNAs in Age-Related Macular Degeneration: An In vivo Animal and Human Study. Front Pharmacol, 2017. 8: p. 168.

116. Blasiak, J., et al., Expression of VEGFA-regulating miRNAs and mortality in wet $A M D$. J Cell Mol Med, 2019. 23(12): p. 8464-8471.

117. Turchinovich, A., et al., Characterization of extracellular circulating microRNA. Nucleic Acids Res, 2011. 39(16): p. 7223-33.

118. Zhang, J., et al., Exosome and exosomal microRNA: trafficking, sorting, and function. Genomics Proteomics Bioinformatics, 2015. 13(1): p. 17-24.

119. Pogue, A.I. and W.J. Lukiw, Up-regulated Pro-inflammatory MicroRNAs (miRNAs) in Alzheimer's disease (AD) and Age-Related Macular Degeneration (AMD). Cell Mol Neurobiol, 2018. 38(5): p. 1021-1031.

120. Kiel, C., et al., A Circulating MicroRNA Profile in a Laser-Induced Mouse Model of Choroidal Neovascularization. Int J Mol Sci, 2020. 21(8). 
121. Zhang, L., et al., Differential Expressions of microRNAs and Transfer RNAderived Small RNAs: Potential Targets of Choroidal Neovascularization. Curr Eye Res, 2019. 44(11): p. 1226-1235.

122. Guo, L., et al., Targeting amyloid-beta in glaucoma treatment. Proc Natl Acad Sci U S A, 2007. 104(33): p. 13444-9.

123. Fisichella, V., et al., TGF- $\beta 1$ prevents rat retinal insult induced by amyloid- $\beta$ (142) oligomers. Eur J Pharmacol, 2016. 787: p. 72-7.

124. Keenan, T.D. and E.Y. Chew, Association Between C-Reactive Protein and AgeRelated Macular Degeneration: Les Liaisons Dangereuses. JAMA Ophthalmol, 2017. 135(9): p. 916-917.

125. Anderson, D.H., et al., Characterization of beta amyloid assemblies in drusen: the deposits associated with aging and age-related macular degeneration. Exp Eye Res, 2004. 78(2): p. 243-56.

126. Zhang, P., et al., MicroRNA-155 Inhibits Polarization of Macrophages to M2Type and Suppresses Choroidal Neovascularization. Inflammation, 2018. 41(1): p. 143-153.

127. Zhuang, Z., et al., Down-regulation of microRNA-155 attenuates retinal neovascularization via the PI3K/Akt pathway. Mol Vis, 2015. 21: p. 1173-84.

128. Wang, S., et al., The endothelial-specific microRNA miR-126 governs vascular integrity and angiogenesis. Dev Cell, 2008. 15(2): p. 261-71.

129. Wang, L., et al., miR-126 Regulation of Angiogenesis in Age-Related Macular Degeneration in CNV Mouse Model. Int J Mol Sci, 2016. 17(6).

130. Zhou, Q., et al., let-7 Contributes to Diabetic Retinopathy but Represses Pathological Ocular Angiogenesis. Mol Cell Biol, 2017. 37(16).

131. Saeedi, P., et al., Global and regional diabetes prevalence estimates for 2019 and projections for 2030 and 2045: Results from the International Diabetes Federation Diabetes Atlas, 9(th) edition. Diabetes Res Clin Pract, 2019. 157: p. 107843.

132. Cho, N.H., et al., IDF Diabetes Atlas: Global estimates of diabetes prevalence for 2017 and projections for 2045. Diabetes Res Clin Pract, 2018. 138: p. 271-281.

133. Yau, J.W., et al., Global prevalence and major risk factors of diabetic retinopathy. Diabetes Care, 2012. 35(3): p. 556-64.

134. Lopez-Galvez, M.I., F. Manco Lavado, and J.C. Pastor, Diabetic Retinopathy, in Handbook of Nutrition, Diet and the Eye. 2014. p. 41-51.

135. Cheung, N., P. Mitchell, and T.Y. Wong, Diabetic retinopathy. Lancet, 2010. 376(9735): p. 124-36.

136. Cai, X. and J.F. McGinnis, Diabetic Retinopathy: Animal Models, Therapies, and Perspectives. J Diabetes Res, 2016. 2016: p. 3789217.

137. Rakoczy, E.P., et al., Characterization of a mouse model of hyperglycemia and retinal neovascularization. Am J Pathol, 2010. 177(5): p. 2659-70.

138. Lechner, J., O.E. O'Leary, and A.W. Stitt, The pathology associated with diabetic retinopathy. Vision Res, 2017. 139: p. 7-14.

139. Kusuhara, S., et al., Pathophysiology of Diabetic Retinopathy: The Old and the New. Diabetes Metab J, 2018. 42(5): p. 364-376.

140. Tarr, J.M., et al., Pathophysiology of diabetic retinopathy. ISRN Ophthalmol, 2013. 2013: p. 343560.

141. Giacco, F. and M. Brownlee, Oxidative stress and diabetic complications. Circ Res, 2010. 107(9): p. 1058-70. 
142. Rübsam, A., S. Parikh, and P.E. Fort, Role of Inflammation in Diabetic Retinopathy. Int J Mol Sci, 2018. 19(4).

143. Miyamoto, K., et al., Prevention of leukostasis and vascular leakage in streptozotocin-induced diabetic retinopathy via intercellular adhesion molecule-1 inhibition. Proc Natl Acad Sci U S A, 1999. 96(19): p. 10836-41.

144. Aiello, L.P., et al., Vascular endothelial growth factor in ocular fluid of patients with diabetic retinopathy and other retinal disorders. N Engl J Med, 1994. 331(22): p. 1480-7.

145. Altmann, C. and M.H.H. Schmidt, The Role of Microglia in Diabetic Retinopathy: Inflammation, Microvasculature Defects and Neurodegeneration. Int J Mol Sci, 2018. 19(1).

146. Wong, T.Y., et al., Guidelines on Diabetic Eye Care: The International Council of Ophthalmology Recommendations for Screening, Follow-up, Referral, and Treatment Based on Resource Settings. Ophthalmology, 2018. 125(10): p. 16081622.

147. Solomon, S.D., et al., Diabetic Retinopathy: A Position Statement by the American Diabetes Association. Diabetes Care, 2017. 40(3): p. 412-418.

148. Royle, P., et al., Pan-retinal photocoagulation and other forms of laser treatment and drug therapies for non-proliferative diabetic retinopathy: systematic review and economic evaluation. Health Technol Assess, 2015. 19(51): p. v-xxviii, 1-247.

149. Bolinger, M.T. and D.A. Antonetti, Moving Past Anti-VEGF: Novel Therapies for Treating Diabetic Retinopathy. Int J Mol Sci, 2016. 17(9).

150. Ferrara, N. and A.P. Adamis, Ten years of anti-vascular endothelial growth factor therapy. Nat Rev Drug Discov, 2016. 15(6): p. 385-403.

151. Sadiq, M.A., et al., Pharmacological agents in development for diabetic macular edema. Int J Retina Vitreous, 2020. 6: p. 29.

152. Martinez, B. and P.V. Peplow, MicroRNAs as biomarkers of diabetic retinopathy and disease progression. Neural Regen Res, 2019. 14(11): p. 1858-1869.

153. Li, X., et al., MicroRNAs: Potential Targets in Diabetic Retinopathy. Horm Metab Res, 2020. 52(3): p. 142-148.

154. Mastropasqua, R., et al., Role of microRNAs in the modulation of diabetic retinopathy. Prog Retin Eye Res, 2014. 43: p. 92-107.

155. Olivares, A.M., et al., Animal Models of Diabetic Retinopathy. Curr Diab Rep, 2017. 17(10): p. 93.

156. Kovacs, B., et al., MicroRNAs in early diabetic retinopathy in streptozotocininduced diabetic rats. Invest Ophthalmol Vis Sci, 2011. 52(7): p. 4402-9.

157. Wu, J.H., et al., Altered microRNA expression profiles in retinas with diabetic retinopathy. Ophthalmic Res, 2012. 47(4): p. 195-201.

158. Gong, Q., et al., Differentially Expressed MicroRNAs in the Development of Early Diabetic Retinopathy. J Diabetes Res, 2017. 2017: p. 4727942.

159. Zhang, J., et al., Downregulation of MicroRNA 29a/b exacerbated diabetic retinopathy by impairing the function of Müller cells via Forkhead box protein O4. Diab Vasc Dis Res, 2018. 15(3): p. 214-222.

160. Zhuang, P., C.K. Muraleedharan, and S. Xu, Intraocular Delivery of miR-146 Inhibits Diabetes-Induced Retinal Functional Defects in Diabetic Rat Model. Invest Ophthalmol Vis Sci, 2017. 58(3): p. 1646-1655.

161. Wang, L., W.X. Liu, and X.G. Huang, MicroRNA-199a-3p inhibits angiogenesis by targeting the VEGF/PI3K/AKT signalling pathway in an in vitro model of diabetic retinopathy. Exp Mol Pathol, 2020. 116: p. 104488. 
162. McArthur, K., et al., MicroRNA-200b regulates vascular endothelial growth factor-mediated alterations in diabetic retinopathy. Diabetes, 2011. 60(4): $\mathrm{p}$. 1314-23.

163. Jayaram, H., et al., MicroRNA Expression in the Glaucomatous Retina. Invest Ophthalmol Vis Sci, 2015. 56(13): p. 7971-82.

164. Loscher, C.J., et al., Altered retinal microRNA expression profile in a mouse model of retinitis pigmentosa. Genome Biol, 2007. 8(11): p. R248.

165. Yu, X., et al., MicroRNAs: new players in cataract. Am J Transl Res, 2017. 9(9): p. 3896-3903.

166. Muraleedharan, C.K., et al., Inactivation of the miR-183/96/182 Cluster Decreases the Severity of Pseudomonas aeruginosa-Induced Keratitis. Invest Ophthalmol Vis Sci, 2016. 57(4): p. 1506-17.

167. Pockar, S., et al., MiRNA as biomarker for uveitis - A systematic review of the literature. Gene, 2019. 696: p. 162-175.

168. Karlstetter, M., et al., Retinal microglia: just bystander or target for therapy? Prog Retin Eye Res, 2015. 45: p. 30-57.

169. Silverman, S.M. and W.T. Wong, Microglia in the Retina: Roles in Development, Maturity, and Disease. Annu Rev Vis Sci, 2018. 4: p. 45-77.

170. Schulz, C., et al., A lineage of myeloid cells independent of Myb and hematopoietic stem cells. Science, 2012. 336(6077): p. 86-90.

171. Santos, A.M., et al., Embryonic and postnatal development of microglial cells in the mouse retina. J Comp Neurol, 2008. 506(2): p. 224-39.

172. Morgan, S.C., D.L. Taylor, and J.M. Pocock, Microglia release activators of neuronal proliferation mediated by activation of mitogen-activated protein kinase, phosphatidylinositol-3-kinase/Akt and delta-Notch signalling cascades. $\mathbf{J}$ Neurochem, 2004. 90(1): p. 89-101.

173. Squarzoni, P., et al., Microglia modulate wiring of the embryonic forebrain. Cell Rep, 2014. 8(5): p. 1271-9.

174. Checchin, D., et al., Potential role of microglia in retinal blood vessel formation. Invest Ophthalmol Vis Sci, 2006. 47(8): p. 3595-602.

175. Wang, X., et al., Requirement for Microglia for the Maintenance of Synaptic Function and Integrity in the Mature Retina. J Neurosci, 2016. 36(9): p. 2827-42.

176. Ma, W., et al., Monocyte infiltration and proliferation reestablish myeloid cell homeostasis in the mouse retina following retinal pigment epithelial cell injury. Sci Rep, 2017. 7(1): p. 8433.

177. O'Koren, E.G., R. Mathew, and D.R. Saban, Fate mapping reveals that microglia and recruited monocyte-derived macrophages are definitively distinguishable by phenotype in the retina. Sci Rep, 2016. 6: p. 20636.

178. D'Orazio, T.J. and J.Y. Niederkorn, A novel role for TGF-beta and IL-10 in the induction of immune privilege. J Immunol, 1998. 160(5): p. 2089-98.

179. Greter, M., et al., Stroma-derived interleukin-34 controls the development and maintenance of langerhans cells and the maintenance of microglia. Immunity, 2012. 37(6): p. 1050-1060.

180. Jurga, A.M., M. Paleczna, and K.Z. Kuter, Overview of General and Discriminating Markers of Differential Microglia Phenotypes. Front Cell Neurosci, 2020. 14: p. 198.

181. Garden, G.A. and T. Moller, Microglia biology in health and disease. J Neuroimmune Pharmacol, 2006. 1(2): p. 127-37. 
182. Karperien, A., H. Ahammer, and H.F. Jelinek, Quantitating the subtleties of microglial morphology with fractal analysis. Front Cell Neurosci, 2013. 7: p. 3.

183. Zanier, E.R., et al., Shape descriptors of the "never resting" microglia in three different acute brain injury models in mice. Intensive Care Med Exp, 2015. 3(1): p. 39 .

184. Glezer, I. and S. Rivest, Glucocorticoids: protectors of the brain during innate immune responses. Neuroscientist, 2004. 10(6): p. 538-52.

185. Pearson, P.A., et al., Fluocinolone acetonide intravitreal implant for diabetic macular edema: a 3-year multicenter, randomized, controlled clinical trial. Ophthalmology, 2011. 118(8): p. 1580-7.

186. Ip, M.S., et al., A randomized trial comparing the efficacy and safety of intravitreal triamcinolone with observation to treat vision loss associated with macular edema secondary to central retinal vein occlusion: the Standard Care vs Corticosteroid for Retinal Vein Occlusion (SCORE) study report 5. Arch Ophthalmol, 2009. 127(9): p. 1101-14.

187. Du, Y., et al., Minocycline prevents nigrostriatal dopaminergic neurodegeneration in the MPTP model of Parkinson's disease. Proc Natl Acad Sci U S A, 2001. 98(25): p. 14669-74.

188. Bosco, A., et al., Reduced retina microglial activation and improved optic nerve integrity with minocycline treatment in the DBA/2J mouse model of glaucoma. Invest Ophthalmol Vis Sci, 2008. 49(4): p. 1437-46.

189. Zhao, L., et al., Minocycline attenuates photoreceptor degeneration in a mouse model of subretinal hemorrhage microglial: inhibition as a potential therapeutic strategy. Am J Pathol, 2011. 179(3): p. 1265-77.

190. Cukras, C.A., et al., Oral minocycline for the treatment of diabetic macular edema (DME): results of a phase I/II clinical study. Invest Ophthalmol Vis Sci, 2012. 53(7): p. 3865-74.

191. Fazi, F., et al., A minicircuitry comprised of microRNA-223 and transcription factors NFI-A and C/EBPalpha regulates human granulopoiesis. Cell, 2005. 123(5): p. 819-31.

192. Forrest, A.R., et al., Induction of microRNAs, mir-155, mir-222, mir-424 and mir503 , promotes monocytic differentiation through combinatorial regulation. Leukemia, 2010. 24(2): p. 460-6.

193. Pospisil, V., et al., Epigenetic silencing of the oncogenic miR-17-92 cluster during PU.1-directed macrophage differentiation. EMBO J, 2011. 30(21): p. 4450-64.

194. Ponomarev, E.D., et al., MicroRNA-124 promotes microglia quiescence and suppresses EAE by deactivating macrophages via the C/EBP- $\alpha-P U .1$ pathway. Nat Med, 2011. 17(1): p. 64-70.

195. Ponomarev, E.D., T. Veremeyko, and H.L. Weiner, MicroRNAs are universal regulators of differentiation, activation, and polarization of microglia and macrophages in normal and diseased CNS. Glia, 2013. 61(1): p. 91-103.

196. Guedes, J., A.L. Cardoso, and M.C. Pedroso de Lima, Involvement of microRNA in microglia-mediated immune response. Clin Dev Immunol, 2013. 2013: p. 186872.

197. Kong, H., et al., Inhibition of miR-181a-5p reduces astrocyte and microglia activation and oxidative stress by activating SIRT1 in immature rats with epilepsy. Lab Invest, 2020. 100(9): p. 1223-1237. 
198. Dong, N. and Y. Wang, MiR-30a Regulates S100A12-induced Retinal Microglial Activation and Inflammation by Targeting NLRP3. Curr Eye Res, 2019. 44(11): p. 1236-1243.

199. Li, G., et al., Exosomes-carried microRNA-26b-5p regulates microglia M1 polarization after cerebral ischemia/reperfusion. Cell Cycle, 2020. 19(9): p. 10221035.

200. Gupta, N., et al., miR-142-3p Regulates BDNF Expression in Activated Rodent Microglia Through Its Target CAMK2A. Front Cell Neurosci, 2020. 14: p. 132. 


\section{Chapter 2: Resistance to Retinopathy Development in Obese, Diabetic and Hypertensive ZSF1 rats: an exciting model to identify protective genes}

Vincenza Caolo*1, Quentin Roblain $^{2,3}$, Julie Lecomte ${ }^{3}$, Paolo Carai ${ }^{1}$, Linsey Peters ${ }^{2}$, Ilona Cuijpers ${ }^{1,2}$, Emma Louise Robinson ${ }^{2}$, Kasper Derks ${ }^{4}$, Jurgen Sergeys ${ }^{5}$, Agnès Noël ${ }^{3}$, Elizabeth A.V. Jones ${ }^{1}$, Lieve Moons ${ }^{5}$ \& Stephane Heymans ${ }^{1,2,} 6$

${ }^{1}$ Department of Cardiovascular Sciences, Centre for Molecular and Vascular Biology, KU Leuven, Belgium.

${ }^{2}$ Department of Cardiology, CARIM School for Cardiovascular Diseases Faculty of Health, Medicine and Life Sciences, Maastricht University, The Netherlands.

${ }^{3}$ Laboratory of Tumor and Development Biology, GIGA-Cancer, University of Liège, Liège, Belgium.

${ }^{4}$ Department of Genetics and Cell Biology, CARIM School for Cardiovascular Diseases Faculty of Health, Medicine and Life Sciences, Maastricht University, The Netherlands.

${ }^{5}$ Laboratory of Neural Circuit Development and Regeneration, Animal Physiology and Neurobiology Section, Department of Biology, KU Leuven, Leuven, Belgium.

${ }^{6}$ The Netherlands Heart Institute, Nl-HI, Utrecht, The Netherlands

Scientific Reports, 2018 Aug 09; 8:11922 


\begin{abstract}
Diabetic retinopathy (DR) is one of the major complications of diabetes, which eventually leads to blindness. Up to date, no animal model has yet shown all the comorbidities often observed in DR patients. Here, we investigated whether obese 42 weeks old ZSF1 rat, which spontaneously develops diabetes, hypertension and obesity, would be a suitable model to study DR.

Although arteriolar tortuosity increased in retinas from obese as compared to lean (hypertensive only) ZSF1 rats, vascular density pericyte coverage, microglia number, vascular morphology and retinal thickness were not affected by diabetes. These results show that, despite high glucose levels, obese ZSF1 rats did not develop DR. Such observations prompted us to investigate whether the expression of genes, possibly able to contain DR development, was affected. Accordingly, mRNA sequencing analysis showed that genes (i.e. Npy and crystallins), known to have a protective role, were upregulated in retinas from obese ZSF1 rats.

Lack of retina damage, despite obesity, hypertension and diabetes, makes the 42 weeks of age ZSF1 rats a suitable animal model to identify genes with a protective function in DR. Further characterisation of the identified genes and downstream pathways could provide more therapeutic targets for the treat DR.
\end{abstract}




\section{Introduction}

Globally, prevalence of diabetes has nearly doubled from $4.7 \%$ in 1980 to $8.5 \%$ of the adult population in 2014 [1]. Diabetes can lead to several complications affecting the cardiovascular system, kidneys, nerves, and eyes [2]. Diabetic retinopathy (DR) is one of the most serious complications of diabetes. Prolonged exposure to high blood glucose levels (hyperglycemia) results in severe damage of the retinal vasculature [2-4]. This can lead to blurred vision, dark spots, flashing lights, and eventually total loss of vision. DR is the leading cause of blindness [5] and makes a big contribution to the total $11.6 \%$ of annual health-care costs accounted for diabetes [6].

Currently, several animal models are being used to study the progression of DR, for example the streptozotocin (STZ)-induced and Akimba mouse models (reviewed in [3]). All these models, however, present serious limitations and they do not reflect all stages of DR progression in humans. In addition, these models do not develop diabetes spontaneously, but are either chemically or genetically induced. For instance, STZ is a toxic substance that is injected in mice or rats to destroy the pancreatic $\beta$-cells $[3,7]$. The Akimba mouse develops DR due to the presence of the human Vascular Endothelial Growth Factor isoform 165 (hVEGF 165 ) transgene, which induces an overexpression of VEGF in photoreceptors [8]. Among the few established animal models that develop diabetes spontaneously, the Zucker Diabetic Fatty (ZDF) rat represents an established model for type 2 diabetes. However, ZDF rats did not show any clear sign of DR, i.e. vascular occlusion or regression [9]. Besides the ZDF, the obese ZDF/Spontaneously Hypertensive Heart Failure (SHHF) F1 hybrid (ZSF1) rat, which are the result of a cross between a ZDF female and a SHHF male, develops metabolic complications typical of type- 2 diabetes and have a more severe phenotype than the ZDF parental strain. Both obese and lean control ZSF1 rats are hypertensive [10]. However, the obese ZSF1 rats are also affected by diabetic nephropathy (DN), insulin resistance, obesity, hyperinsulinemia, hypercholesterolemia, congestive heart failure, and hypertriglyceridemia [11]. The ZSF1 rat is currently used as a model to study DN, whereas no scientific study on DR performed in these animals has been reported to date. In this study, we extensively investigated whether the ZSF1 rat could represent a suitable animal model to study the pathogenesis of DR. In order to assess the retinal vascular changes caused by diabetes, such as arteriolar tortuosity, obese and lean control ZSF1 rats were subjected to Heidelberg Retina Angiography (HRA) and additional histological analysis over a period of 42 weeks. We further examined the potential combined effect of chronic diabetes, obesity and hypertension on vasculature of retinas isolated from 6 and 42 weeks old obese and lean control ZSF1 rats, by assessing vascular density, pericyte coverage and number of microglia on whole mounted retina. The thickness of the neural retinal layers was assessed by Optical Coherence Tomography (OCT) over a period of 35 weeks. However, no differences were detected between obese and lean ZSF1 rats.

Despite the increase in vascular tortuosity, obese ZSF1 rats did not develop DR. The absence of a DR phenotype would suggest the existence of a protective gene expression profile in these rats. Consistently, deep sequencing analysis of mRNA isolated from retinas 
of 6 and 42 weeks old obese and lean ZSF1 rats, revealed the upregulation of several genes with a potential protective function, i.e. Neuropeptide Y (Npy) and several crystallin genes, at 42 weeks. Whereas genes, previously described to drive vascular inflammation, such as Intercellular Adhesion Molecule 1 (Icaml) and Toll-like receptor 4 (Tlr4) were downregulated in retinas from obese rats 42 weeks of age.

A deeper understanding on the role of Npy and the crystallin genes in the retina following stressful conditions such as diabetes could be beneficial for developing better tools to improve the condition of patients affected by DR and other diabetes related ocular complications. 


\section{Results}

In the clinic, arteriolar tortuosity is routinely used as a marker to predict pathological neovascularization and rapid DR progression [12]. Therefore, we assessed arteriolar tortuosity index in lean and obese ZSF1 rats over a period of 6 to 42 weeks of age. As shown in figure 1 , the tortuosity index significantly increased in obese rats compared to their lean controls at 18, 26, 34 and 42 weeks, but did not differ when comparing lean and obese ZSF1 rats at 5 and 14 weeks of age. Increase in weight and blood glucose levels was confirmed in obese ZSF1 rats at 18, 22 and 42 weeks as reported in Supplementary figure $1 \mathrm{~A}$ and B.

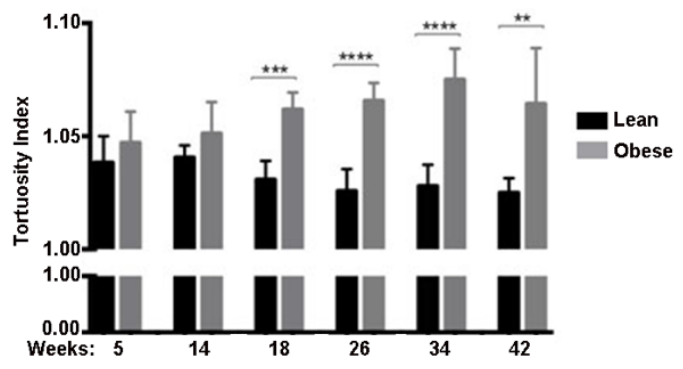

Figure 1. Arteriolar tortuosity is increased in ZSF1 rats over time

Arteriolar tortuosity index of the retinal vasculature from lean and obese ZSF1 rats, between 5 and 42 weeks old. Tortuosity index of the retinal arteries was increased in obese as compared to leans ZSF1 rat retinas of 18 , 26,34 and 42 weeks of age. No difference in arteriolar tortuosity was detected in rats of 5 and 14 weeks of age. All values are mean $+/-\mathrm{SD},{ }^{*} \mathrm{P}<0.01 ; * * * \mathrm{P}<0.001 ; * * * \mathrm{P}<0,0001$.

Besides vessel tortuosity, two characteristics of DR are pericyte dropout and pathological neovascularization. Specifically, pericyte dropout leads to capillary occlusions followed by hypoxia $[13,14]$. In the initial phase, blood vessels retract due to vessel instability caused by the loss of pericytes [15]. This leads to hypoxic areas, which in turn increases the expression of HIF-1 and subsequent VEGFA, resulting in pathological neovascularisation $[16,17]$. Remarkably, deep and superficial vascular density and pericyte coverage did not differ between lean and obese animals at 6 and 42 weeks of age, but also there was no time-induced difference in obese and lean control ZSF1 rats at 42 weeks compared to those at 6 weeks (figure 2B-C). These results suggest that the vascular density and pericyte coverage are not affected by both, age and diabetes in ZSF1 rats. 
A
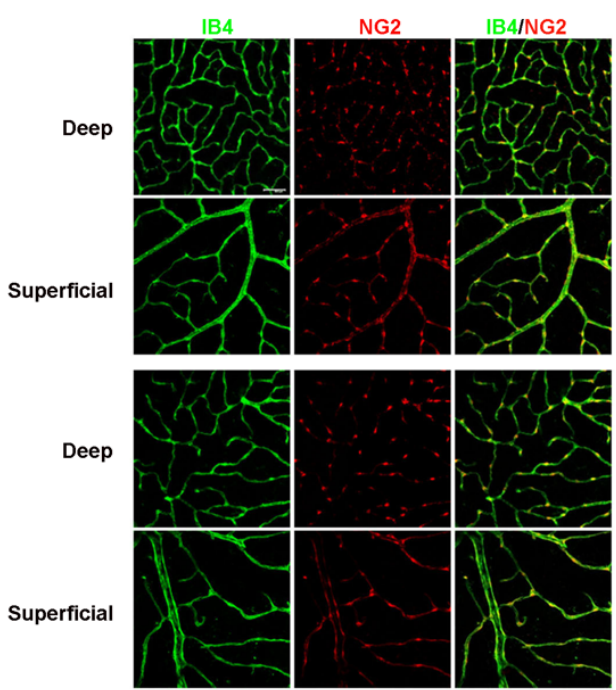

Obese

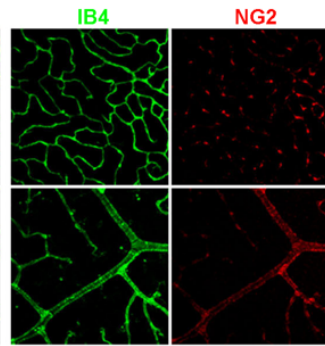

IB4/NG2
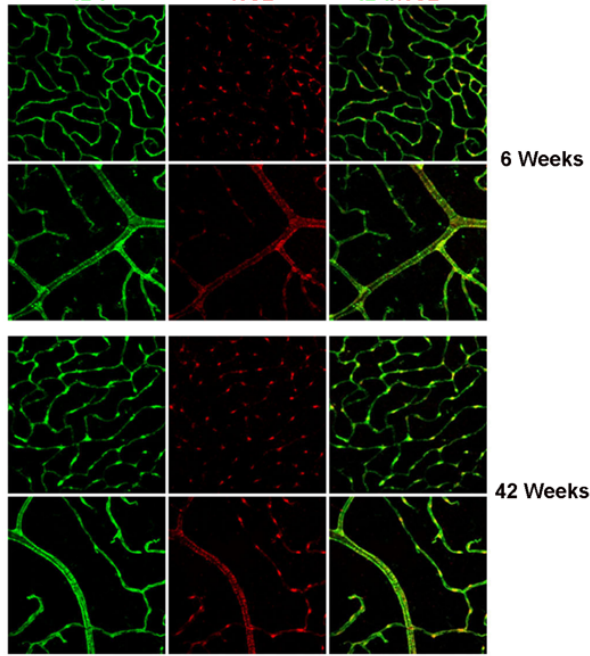

B
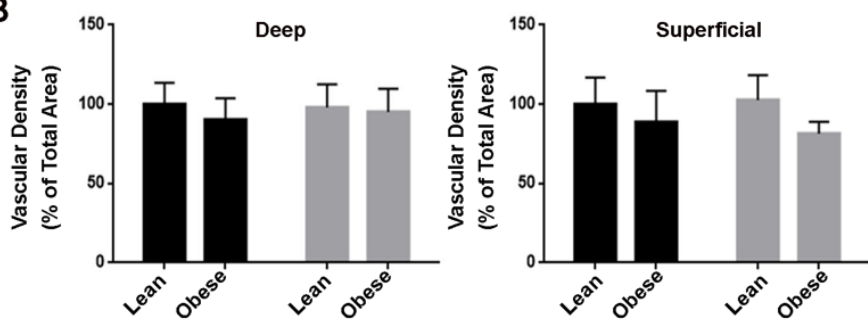

C

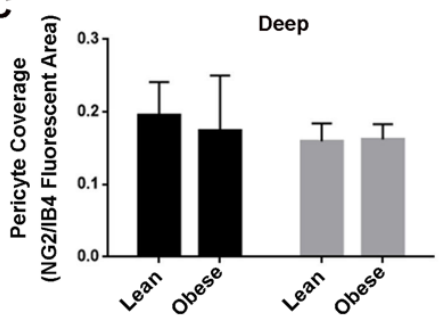

6 Weeks

42 Weeks

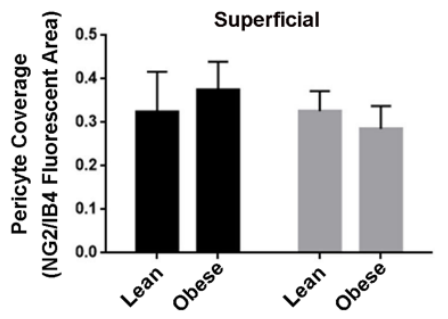

6 Weeks

42 Weeks

Figure 2. Retinal vascular density and pericyte coverage are not affected by diabetes or age.

Vascular density and pericyte coverage of retinal vasculature from 6 and 42 weeks old lean and obese ZSF1 rats. (A) IB4 (green), NG2 (red) and a merge of signal of both the deep and superficial retinal vascular plexus. Scale $=50 \mu \mathrm{m}$. (B) Quantification of vascular density of the deep and superficial plexus shown in percentage of IB4 signal of the total area ( $n=6-7$ per data point). (C) Quantification of pericyte coverage of the deep and superficial plexus shown as ration of the NG2 and IB4 positive areas ( $n=5-7$ per data point). All values are mean $+/-$ SD. 
The activation of several signalling pathways during DR results in increased inflammation [4]. Microglia constitute the resident immune cells of the central nervous system [18] and they seem to play an important role during DR progression [19-21]. The number of Iba1-stained microglia was significantly higher in the retinas of both lean and obese ZSF1 rats at 42 weeks as compared to lean and obese ZSF1 rat at 6 weeks (figure 3A$\mathrm{B})$, which is in line with an age-dependent increase [21]. However, the number of microglia did not differ between lean and obese ZSF1 either at 6 and 42 weeks of age (figure 3B), indicating that diabetes did not impact the microglia cell number in obese ZSF1 rat retinas.

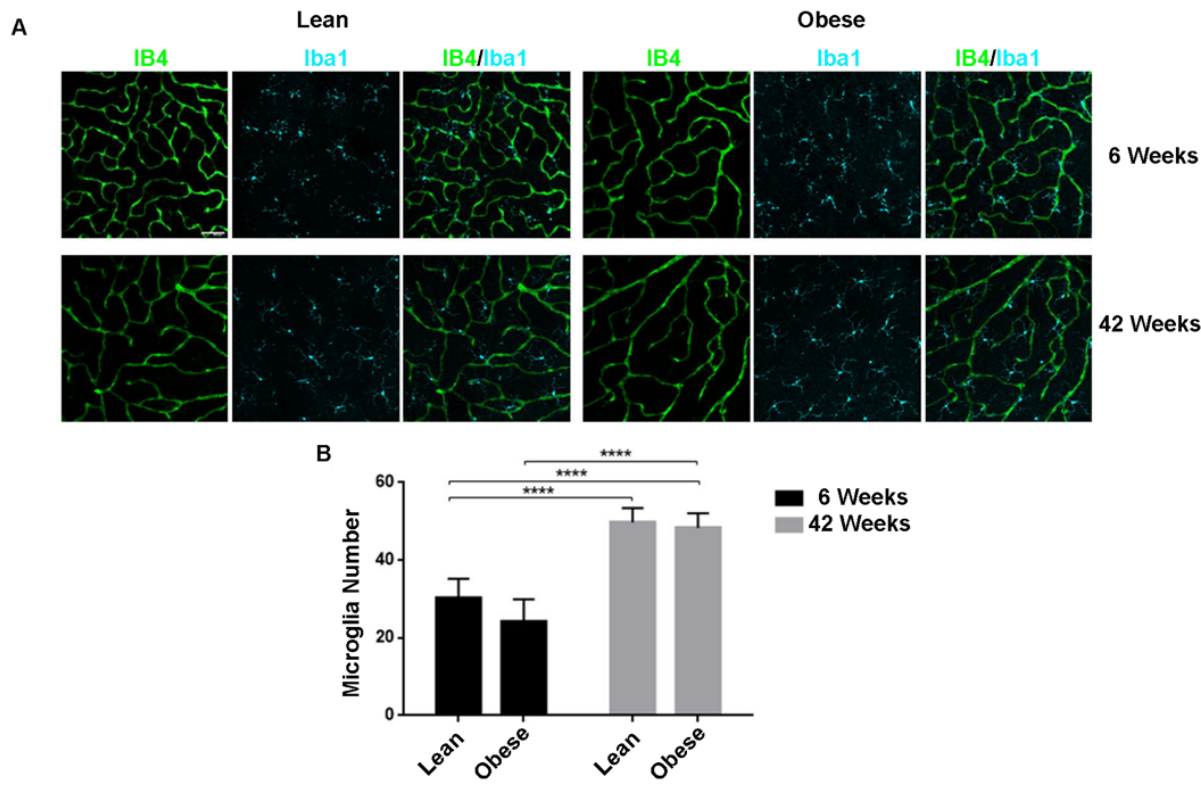

Figure 3. The number of microglia is affected by age, but not diabetes.

Number of microglia located in the retinas from 6 and 42 weeks old lean and obese ZSF1 rats. (A) IB4 (green) and Iba1 (cyan) and merge signal of the deep retinal vascular plexus. Scale $=50 \mu \mathrm{m}$. (B) Quantification of microglia number counted in the intermediate and deep vascular plexus $(n=5-7$ per data point). All values are mean $+/-\mathrm{SD}, * * * *<<0,0001$. 
Next, we determined whether obese ZSF1 rats showed any changes in the morphology of their retinal vascular network, stained with IB4, as compared to their lean controls. An illustration of junctions, segments, loops and branches is reported in Supplementary figure 2. In the deep retinal vascular plexus, the number of junctions, segments, loops and branches were significantly decreased in 42 weeks old lean and obese rats as compared to the 6 weeks old lean and obese ZSF1 rats, respectively (figure 4A-D; Deep). Also, in the superficial vascular plexus, the number of junctions, segments and branches were significantly decreased in the 42 weeks old obese ZSF1 rats compared 6 weeks old obese ZSF1 rats (figure 4A, B, D; Superficial). No significant difference was found in the number of loops in the superficial plexus between the groups (figure 4C; Superficial). Finally, no significant difference was found in the number of junctions, segments, branches and loops in the superficial and deep plexus of retinal vasculature between lean and obese ZSF1 rats at 6 and 42 weeks respectively. Overall, these results indicate that the vascular morphology in the retina is affected by age, but not by diabetes in both deep and superficial plexus. Functional analysis, i.e. OCT scan, of the retina was also performed. As shown in Supplementary figure 3 , no differences in neural retina were found between obese and lean rats. 
A

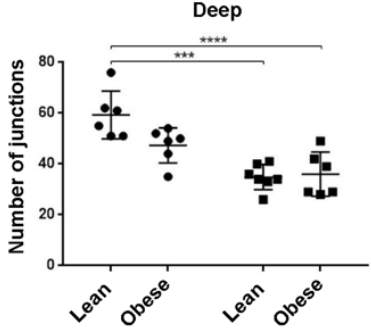

B

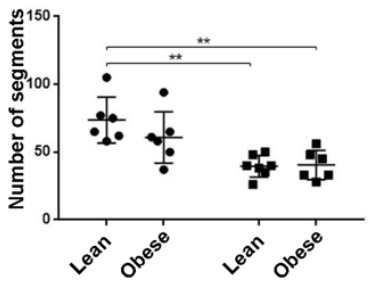

C

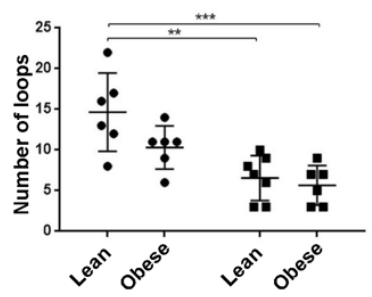

D

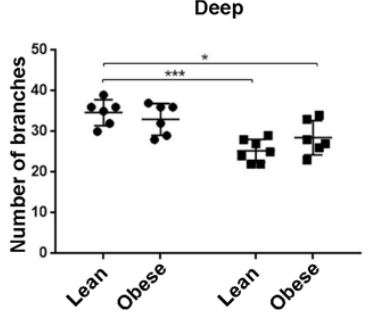

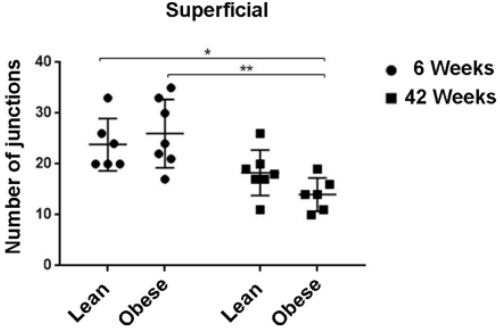

Superficial

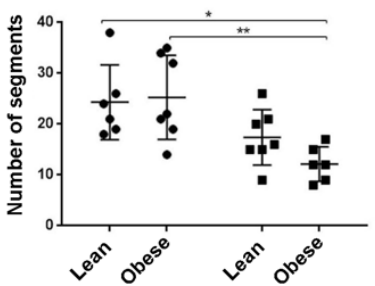

Superficial

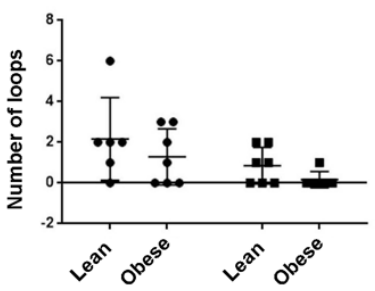

Superficial

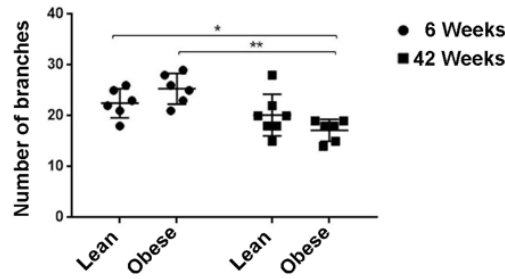

Figure 4. The morphology of the vasculature is affected by age, but not diabetes. Quantification of vascular network morphology of the vascular deep and superficial plexus of retinas from 6 and 42 weeks old lean and obsess ZSF1 rats. Number of junction (A), segments (B), loops (C) and branches (D) in the deep and superficial retinal vascular plexus ( $\mathrm{n}=5-7$ per data point). All values are mean $+/-\mathrm{SD}, * \mathrm{P}<0.05$; $* * \mathrm{P}<0.01 ; * * * \mathrm{P}<0.001 ; * * * * \mathrm{P}<0,0001$. 
To identify genes able to prevent the onset of DR in obese ZSF1 rat, we performed sequencing of mRNA of retinas from 6 and 42 weeks old lean and obese, ZSF1 rats. Principal Component Analysis (PCA) resulted in a clear separation of lean from obese ZSF1 rats (figure 5). Although less pronounced, a separation based on age could be also distinguished within the lean and obese groups, i.e. 6 versus 42 weeks old lean and 6 versus 42 weeks old obese ZSF1 rats (figure 6).

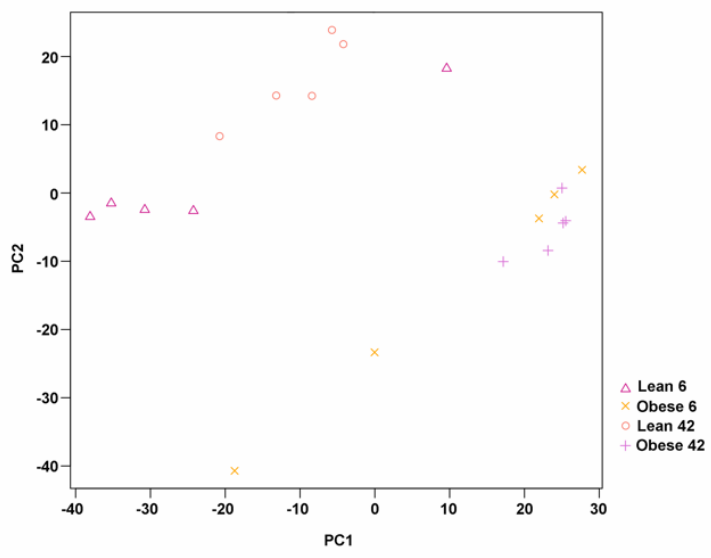

Figure 5. Principal component analysis.

Principal component analysis (PCA) plot based on the first two (PC1-PC2) components of mRNA from retinas from 6 and 42 weeks old lean and obese ZSF1 rats.

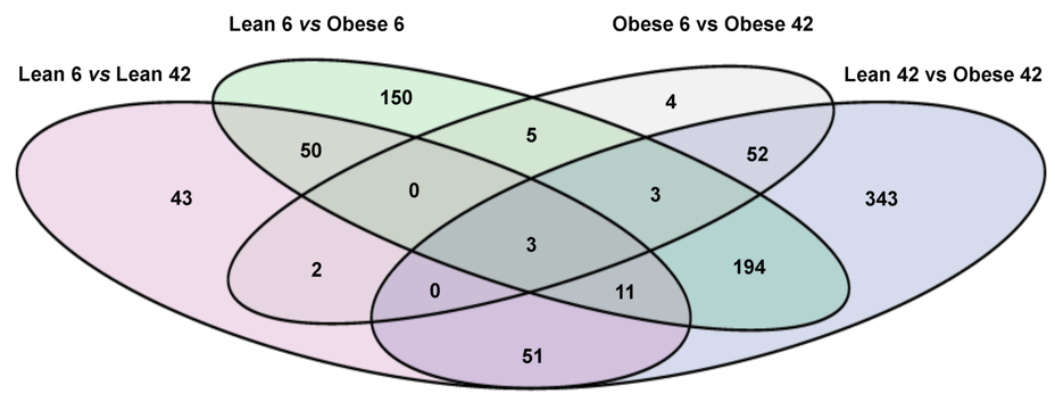

Figure 6. Venn diagram.

Venn diagram showing overlap between the differentially expressed genes (DEG) found in different comparisons, i.e. by age (Lean 6 vs Lean 42, purple and Obese 6 vs Obese 42, gray) and diabetes and metabolic syndrome (Lean 6 vs Obese 6, green and Lean 42 vs Obese 42, blue). The cut-offs for the DEGs were set to a Fold Change $<$ or $>1,5$ and FDR $<0,05$. The numbers in the circle depict the number of DEGs found for that specific condition: i.e. in the bottom circle 51 DEGs are found in both Lean 6 vs Lean 42 and Lean 42 vs Obese 42 . 
We performed statistical analysis on all genes expressed between the different groups. A gene was considered to be expressed when at least 5 reads were found aligned in at least all samples of one of the groups. In figure 6, we report the differential expressed genes (DEGs) found for all the comparisons. Changes in the expression of a total of 911 protein coding genes were identified $(\mathrm{FC}>1.5$ False Discovery Rate $(\mathrm{FDR})<0.05)$. A large majority of the DEGs identified per comparison was unique to that specific comparison, i.e. the DEGs were not significantly regulated in the other comparisons. Ingenuity Pathway Analysis (IPA) analysis showed the differently regulated pathways within the 4 comparisons: lean vs obese 6 weeks old, lean vs obese 42 weeks old, lean 6 vs lean 42 weeks old, obese 6 vs obese 42 weeks old (Supplementary table 1 to 4 ). Noteworthy, the leptin signalling pathway appeared to be differently regulated in the obese 6 and obese 42 weeks old (Supplementary table 4), confirming the metabolic phenotype affecting old obese ZSF1 rats (Supplements, table 4).

Among genes only upregulated in the retinas from 42 weeks old ZSF1, we found Versican (Vcan), Forkhead Box D1 (Foxd1), MHC Class I Polypeptide-Related Sequence B (Micb) and Npy, whereas Cxadrl1, as Immunoglobulin Superfamily Member 11 (IGSF11), and Mal-like (Mall) were downregulated (Figure 7). Remarkably, among the top regulated genes in retina from 6 vs 42 weeks old obese ZSF1, were several members of the crystallin gene family (Figure 8). Among them, Crybb3, Cryba2, Crygc, Crygb, Crygf, Crybb2, Crybal, Cryba4, Crygs, were upregulated in obese 42 as compared to obese 6 weeks old. However, Crybb3, Cryba2, Crygc, Crygb, Crygf, Crybal were only upregulated in obese 42, indicating that their expression was likely affected by diabetes and metabolic diseases. On the other hand, Icam 1 and Trl4, both known to play a determinant role during vascular inflammation, were both downregulated in retinas from obese rat 42 weeks old (figure 9).

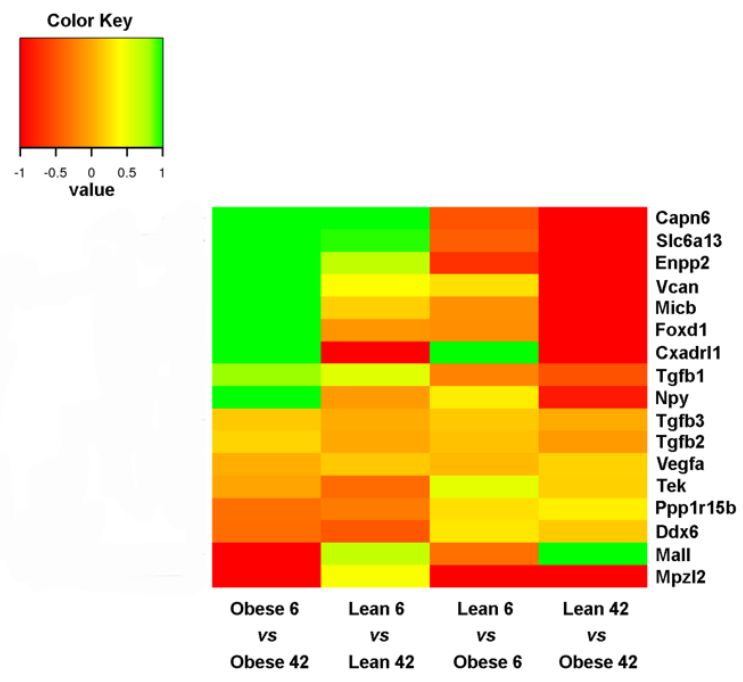

Figure 7. Heat map showing upregulation of several genes

Heat map displaying fold changes of the differential expressed genes. The colors are ranging from red $(-1<=$ $\log 2$ Fold Change $(\mathrm{FC}))$ to orange $(\mathrm{FC} \sim 0)$ to green $(\mathrm{FC}>=1)$. 


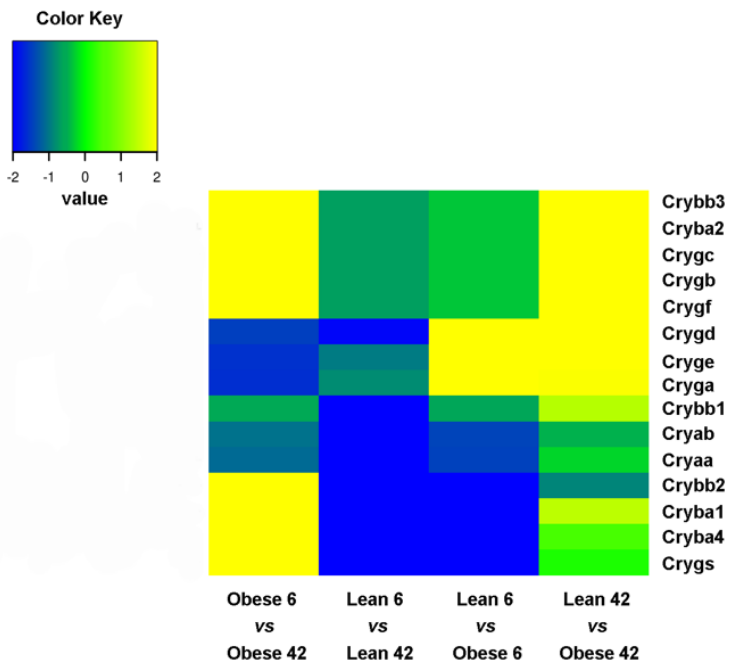

Figure 8. Heat map showing upregulation of crystallin gene family

Heat map displaying fold changes of the differential expressed crystallin (Crys) genes. The colors are ranging from blue $(-2<=\log 2$ Fold Change $(\mathrm{FC}))$ to green $(\mathrm{FC} \sim 0)$ to yellow $(\mathrm{FC}>=2)$.

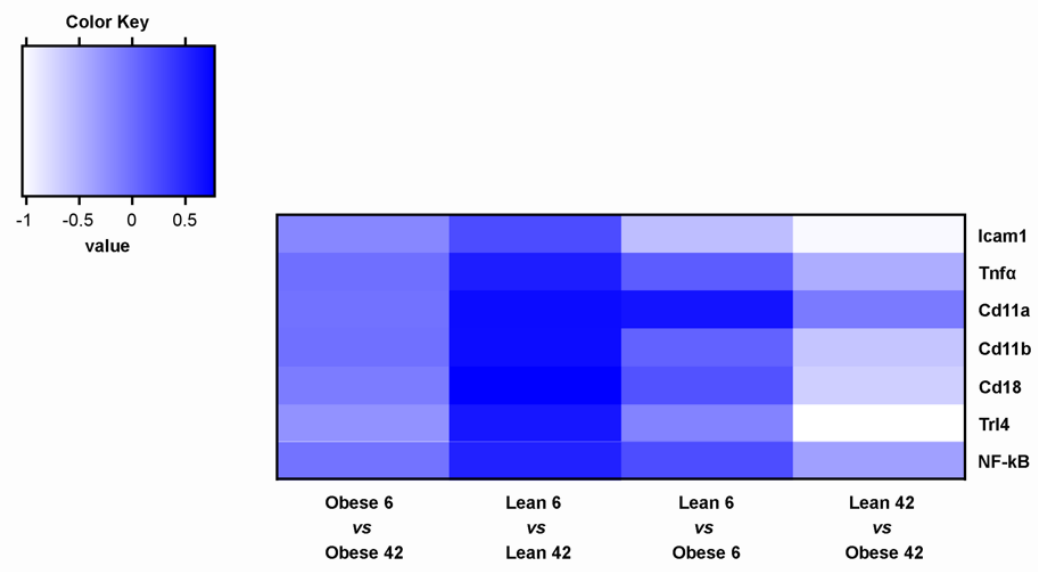

Figure 9. Heat map showing upregulation of indicated genes

Heat map displaying fold changes for indicated genes. The colors are ranging from white $(-1<=\log 2$ Fold Change $(\mathrm{FC})$ ) to light blue $(\mathrm{FC} \sim 0)$ to dark blue/violet $(\mathrm{FC}>=1)$. 


\section{Discussion}

The ZSF1 rat, despite being obese, hypertensive, diabetic and hyperlipidemic, did not present any clear vascular defects in their retinas that could be attributed to progressive DR. Although vascular tortuosity increased in the diabetic rats over time, vascular density and pericyte coverage did not differ between diabetic and non-diabetic rats of 6 and 42 weeks of age. Microglia number and vascular network morphology differed in aged rats, but were unaffected by diabetes. Thickness of neural retina layers were also assessed by OCT scan, however no differences were found at the different analysed time points, in lean and obese rats. Absence of damage in the retinas from diabetic aged ZSF1 rats offered us the existing opportunity to investigate on possible molecular mechanisms that would prevent these animals from developing DR. We, therefore, performed mRNA sequencing in order to identify genes differentially expressed in retina isolated from lean and obese ZSF1 rats 6 and 42 weeks of age with a possible protective role against DR.

The RNA sequencing revealed that 85 genes were upregulated in the retinas from 42 weeks old when compared to their lean control ZSF1 rats. Among the regulated genes, we found genes known to play a role in angiogenesis, inflammation, cellular stress and death. We found that genes involved in the process of angiogenesis, i.e. VCAN and Foxd1, were upregulated. In addition, the expression of the Micb gene, whose increase has been associated with degeneration of retinal ganglionic cells (RGC) and development of glaucoma [22], was upregulated in retinas from 42 weeks old ZSF1 rats. On the other hand, Cxadrl1, important for excitatory synaptic localization and plasticity, and Mall, encoding a component of raft mediated trafficking in endothelial cells, were both downregulated. Although changes in expression of the above mentioned genes, together with increased vascular tortuosity, would suggest that an initial vascular and neuronal damage of the retinas in ZSF1 rats 42 weeks of age is occurring, we also found that several genes with a potential protective function, i.e. Npy and several crystallin genes, were significantly upregulated in retinas from 42 weeks old ZSF1 rats. Conversely, genes involved in vascular inflammation, like Icam1 and Trl4 [23, 24], were downregulated in in retinas from 42 weeks old ZSF1 rats.

Npy was first isolated in 1982 and is highly conserved between species [25]. In the retina, Npy seems to be required during development being expressed in different cell types [26]. Specifically, the neuroprotective role of Npy against excitotoxicity has been largely documented [27-29]. As such, Npy was shown to reduce the $\left[\mathrm{Ca}^{2+}\right]_{\mathrm{i}}$ increase in rat neurons preventing their death [30]. The activation of several Npy receptors has been reported to protect retinal cells from necrosis or glutamate induced cell death [31]. Npy and its receptors are expressed in retinal endothelial cells, likely playing a role in retinal related vascular diseases [30]. In support of this, few studies have shown that Leucine to Proline polymorphism in the Npy gene was related to an increased predisposition to develop diabetic retinopathy in type 2 diabetic patients [32-34]. However, results on the role of Npy in oxygen-induced retinopathy model have been controversial. Whereas two studies [33, 35] suggested a positive role for Npy in the progression of neovascularization during DR, Schmid and collaborators [36] reported a decreased expression level of Npy in a similar model. 
Therefore, further research is still needed to clarify the exact contribution of Npy in retinal vascular diseases.

Several crystalline genes were also found to be upregulated in retinas of obese ZSF1 when compared to lean 42 weeks of age. The crystallin genes were first described to encode structural proteins of the lens [37], but they are also expressed in other tissues and organs such as the retina [38], heart [39] and skeletal muscle [40]. The crystallins are divided in two families: the $\alpha$-crystallin belonging to the small heat shock proteins (sHSP) and the $\beta$ - and $\gamma$ crystallins forming the $\beta / \gamma$ superfamily. Besides their function in lens transparency and reflex index, the $\alpha$-crystallins are induced in response to stress and injury, displaying molecular chaperone and anti-apoptotic activity [41]. The $\beta / \gamma$ crystallins regulate vascular remodelling during eye development and RCG axon regeneration [42]. Crystallin gene expression is altered upon DR [38], but their exact function during this process remains controversial. Although few studies have suggested a protective role of $\alpha \mathrm{B}$-crystallin genes against pathological neovascularization of the retina during diabetes, one study reported that delivery by intravitreous injection of recombinant adenovirus expressing $\alpha \mathrm{A}$-crystallin prevented vascular leakage and decreased pericyte loss in STZ-induced mouse model of diabetes [43-46]. The majority of those studies, however, investigated the role of $\alpha$-crystallin genes during DR, whereas little is still known on the role of $\beta / \gamma$ crystallins. From our RNA sequencing analysis, it emerges that diabetes and the metabolic syndrome induce the expression of crystallin $\beta$ (i.e. Crybb3, Cryba2, Cryba1) and $\gamma$ (i.e. Crygc, Crygb, Crygf) in the retina, whereas we did not observe any significant effect on $\alpha$ crystallins.

Noteworthy, $\beta$ and $\gamma$ crystallin genes are upregulated in the retina of Nuc1 mutant rats as compared to wild type littermates [47]. Nuc1 mutants are characterized by a spontaneous mutation that affects neuronal and vascular remodelling and retinal function [48]. In particular, astrocytes at the vascular front seem to express $\beta$ and $\gamma$ crystallins together with VEGF. Moreover, in human persistent fetal vasculature (PFV) disease, in which the hyaloid vasculature does not regress normally, astrocytes expressed $\beta$ and $\gamma$ crystallins [47], suggesting a role for those crystallins in vascular stabilization in the eye. Besides being vasculoprotective, $\beta / \gamma$ crystallins may also modulate axon regeneration [49]. $B / \gamma$ crystallins induce ciliary neurotrophic factor (CNTF) and brain derived neurotrophic factor (BDNF) both in vitro and in vivo [49]. In particular Crybb2 is secreted by cultured retinas during axon regeneration, and induces axon elongation in cultured RCG axons [42]. In conclusion, $\beta / \gamma$ crystallin genes, which are upregulated in the retinas of 42 weeks old obese ZSF1 rats, might play a role in vascular stabilization and neuronal survival, preventing ZSF1 rats from developing DR.

Vascular inflammation and leukostasis are early events in diabetic retinopathy with serious functional consequences. During DR leukocytes adhere to endothelial cells, causing vascular occlusion, macrophage accumulation and vascular tissue damage[50]. Several are the genes involved in these processes. Among them, Tumor Necrosis Factor $\alpha(\operatorname{Tnf} \alpha)$ modulates endothelial cell permeability and adhesion molecule expression [51]. Also, the expression of CD11a, CD11b, and CD18 integrins was shown to be increased on the surface of neutrophils from diabetic rats [50]. Proteins such as, Tnf $\alpha$, NF-kB are Trl4 have been 
extensively shown to regulate the inflammatory response [23]. Finally, Icam1 inhibition was reported to prevent diabetic retinal leukostasis and blood-retinal barrier breakdown [24]. Therefore, we assessed the expression of all these vascular inflammation markers and to our surprise we found that Icam 1 and Trl4 were downregulated in in retinas from 42 weeks old ZSF1 rats, likely contributing to the protective mechanisms by reducing leukostasis and inflammation.

Although the diabetic, hypertensive, obese and hyperlipidemic ZSF1 rat does not develop overt DR, it represents a useful model to identify new molecular signalling pathways beneficial to prevent the onset of DR. Remarkably, RNA sequencing analysis led us to identify $N p y, \beta / \gamma$ crystalline, Icam 1 and Trl4 genes that are differently expressed in lean and obese ZSF1 rats and could play a role in protecting old obese ZSF1 rats from developing DR. The roles that $N p y$ and $\beta / \gamma$ crystallins play in vascular remodelling and axon regeneration, and that Icam 1 and Trl4 play in vascular inflammation, make them interesting candidates for new studies to target a complex ocular disease such as DR. Further characterization of the molecular mechanisms regulated by $\beta / \gamma$ crystallins and Npy, Icam 1 and Trl4, whose activation might be protective against DR, could possibly help us to design novel and more effective therapeutic strategies to improve the clinical outcome of patients affected by DR. 


\section{Methods}

- Animals

All animal procedures conformed to the relevant guidelines and regulations of, and were approved by, the Animal Welfare Committee of the KU Leuven University. Male obese ZSF1 rats (ZSF1-Lepr ${ }^{\text {fa }}$ Lepr $\left.^{\mathrm{cp}} / \mathrm{Crl}\right)$ (Charles River Inc.) were used in this study. Male lean, non-diabetic, non-obese, but hypertensive ZSF1 rats were used as control. Rats were sacrificed at 6 and 42 weeks of age. The weight of each overnight fasting rat was recorded. Diabetes was confirmed after blood collection from rat tails at different time points $(18,22$ and 42 weeks) and glucose levels were determined with an automated glucose analyzer device (Glucometer, Menarini Diagnostics).

\section{- Heidelberg Retinal Angiography}

Rats were anesthetized by intraperitoneal injection of ketamine (Ketalar, $10 \mathrm{mg} / \mathrm{mL}$ ) at a dose of $6.0 \mathrm{mg} / \mathrm{kg}$ and medetomidine hydrochloride (Domitor, $1.0 \mathrm{mg} / \mathrm{mL}$ ) at a dose of 0.4 $\mathrm{mg} / \mathrm{kg}$. Rats were injected intraperitoneally with $1 \mathrm{~mL}$ fluorescein sodium salt (Sigma, F6377-100G) $10 \%$ solution in saline. After the experiment, rats were awaken by intraperitoneal injection of atipamezole hydrochloride (Antisedan, $5 \mathrm{mg} / \mathrm{ml}$ ) at a dose of 0.5 $\mathrm{mg} / \mathrm{kg}$. Images were taken using a Heidelberg Retina Angiograph 2 (HRA2) (Heidelberg Engineering $\mathrm{GmbH}$ ) according to the manufacturer's recommendations. Tortuosity index was calculated from these photographs using the ImageJ software as previously described [52].

\section{- Retina Isolation and Immunofluorescence}

Retina isolation and staining were carried out as previously described [53]. Blood vessels were stained with $20 \mu \mathrm{g} / \mathrm{mL}$ Isolectin GS-IB4 (IB4) (I21411, Thermo Fisher). Pericytes were stained with $5 \mu \mathrm{g} / \mathrm{mL}$ primary antibody Mouse anti-NG2 (Cat. No. NG2 372700 Invitrogen) and microglia were stained with $2 \mu \mathrm{g} / \mathrm{mL}$ primary polyclonal antibody Rabbit anti-Iba1 (019_19741, Wako Laboratory Chemicals). 1:100 Goat anti-Mouse Alexa 568 (A11031, Molecular Probes) and 1:100 Donkey anti-Rabbit Alexa 647 (A31573, Invitrogen) were used as secondary antibodies. Photos were taken using a Leica DFC350 FX digital camera or Leica TCS SPE confocal.

The images were processed and analyzed in ImageJ. The superficial and deep vascular plexus were separated based on the IB4 images. All analyses were performed on 20X magnification images with ImageJ Angiogenesis Analyzer. This tool allows detailed quantification of several vasculature structures, such as number of junctions, segments, loops and branches as shown in Supplementary Figure 2. Junctions were defined as meeting points of segments and/or branches. Segments were described as elements between two junctions and branches are elements between a junction and extremity. Loops were defined as areas enclosed by segments [54]. The percentage of IB4 positive areas was evaluated to determine vascular density. The percentage of NG2 positive areas was measured and the ratio of IB4 and NG2 positive areas was calculated to determine pericyte coverage. For area calculations, 
the images were quantified by ImageJ Area Fraction analysis. Microglia were quantified by ImageJ Cell Counter analysis.

\section{- Optical Coherence Tomography}

To assess thickness of the retinal layers and retinal morphology, a spectral domain optical coherence tomography (SD-OCT) system (Envisu R2210, Bioptigen, Morrisville, NC, USA) was used. To evaluate retinal morphology and neural retinal thickness an InVivoVue Diver 2.2 software (Bioptigen) was used as previously described in [55].

\section{- $\quad$ RNA isolation and integrity}

RNA was isolated from the ZSF1 retinas using the mirVana ${ }^{\mathrm{TM}}$ miRNA Isolation Kit (Cat. No 1560, Ambion) according to the mirVana ${ }^{\mathrm{TM}}$ miRNA Isolation kit protocol. In a 20 $\mu \mathrm{L}$ reaction volume, $1 \mu \mathrm{g}$ RNA per sample was reverse transcribed into cDNA via the miScript II RT Kit (Cat. No. 218160, Qiagen), according to the manufacturer's instructions using the 5x miScript HiFlex Buffer.

The integrity of the RNA from each sample was scored on the Agilent 2100 Bioanalyzer (Agilent) by using an Expert Eukaryote Total RNA Pico chip according manufacturer's protocol. Samples that had an RNA Integrity Number (RIN) value of over 6 were subsequently used for mRNA sequence library generation.

\section{- mRNA sequencing library generation}

The mRNA sequencing library was generated using TruSeq mRNA sample preparation kit (Illumina) according to manufacturer's protocol. In short, mRNA was enriched using magnetic beads coated with poly-dT, followed by fragmentation. The fragmented mRNAenriched samples were subjected to cDNA synthesis by reverse transcriptase, followed by dA-tailing and ligation of specific double-stranded bar-coded adapters. Subsequently, a 15 cycles library amplification was performed and after clean-up, the sizes of the libraries were determined on an Agilent 2100 Bioanalyzer (Agilent) via an DNA 1000 chip according manufacturer's protocol. Pooled libraries consisting of equal molar samples were sequenced on a high-output $75 \mathrm{bp}$ single read on the NextSeq500 (Illumina).

\section{- Total RNA analysis pipeline}

The analyses of sequencing datasets were performed as earlier described [56]. In short, reads were aligned to the rat rn6 reference genome using TopHat [43] and exonic reads were summed per transcript and transcripts were referred to as being expressed when at least five aligned reads were present in all samples of at least one of the groups. 
- Pathway analysis

The differentially transcribed genes for the obese, i.e. 6 and 42 weeks, or lean, i.e. 6 and 42 weeks, ZSF1 rats were investigated for over-represented pathways. We used normalized count reads, i.e. counts per million and $\log 2$ transformed, and performed a pathway enrichment analysis with Ingenuity pathway analysis (IPA) software.

\section{- $\quad$ Statistics}

Statistical analysis was performed by using GraphPad Prism 6. Statistical differences were examined by applying one and two-way analysis of variance tests (ANOVAs) followed by Tukey's Multiple Comparison Test. A $p$ value of less than $0.05(\mathrm{P}<0.05)$ was considered statistically significant. Data are presented as the mean \pm standard deviation (SD) or \pm standard error of the mean (SEM) of 5-7 retinas per group. 


\section{Data availability}

All data generated or analyzed during this study are included in this published article (and its Supplementary Information files).

\section{Acknowledgements}

The research leading to these results has received co-funding from the European Union Commission's Seventh Framework Programme under grant agreement N 305507 (HOMAGE), co-funded by the C3 project "Vision Core Leuven" of the Leuven University. We acknowledge the support from the Netherlands Cardiovascular Research Initiative, an initiative with the support of the Dutch Heart Foundation, CVON2016-Early HFPEF, CVON 2017-ShePREDICTS and ERA-CVD funding (FWO G0H7716N). This project was also supported by a joined $\mathrm{PhD}$ funding program in the framework of the cooperation between Maastricht University and Liege University named "Towards a joint imaging valley".

\section{Author Contributions}

VC conceptualized the work, performed investigations and wrote the manuscript; QR, PC, IC, LP, ELR and KD performed investigations and reviewed/edited the manuscript; JL, AN, EAVJ, LM and JS reviewed/edited the manuscript; SH conceptualized the work, wrote the manuscript and supervised the work.

\section{Competing Interests}

The authors declare no competing financial and non-financial interests. 


\section{References}

1. WHO, Global report on diabetes. 2016: Geneva.

2. Federation, I.D., IDF Diabetes Atlas, 7th edn. 2015: Brussels, Belgium.

3. Cai, X. and J.F. McGinnis, Diabetic Retinopathy: Animal Models, Therapies, and Perspectives. Journal of Diabetes Research, 2016. 2016: p. 3789217.

4. Hendrick, A.M., M.V. Gibson, and A. Kulshreshtha, Diabetic Retinopathy. Primary Care: Clinics in Office Practice, 2015. 42(3): p. 451-464.

5. Lee, R., T.Y. Wong, and C. Sabanayagam, Epidemiology of diabetic retinopathy, diabetic macular edema and related vision loss. Eye and Vision, 2015. 2: p. 17.

6. Zheng, Y., M. He, and N. Congdon, The worldwide epidemic of diabetic retinopathy. Indian Journal of Ophthalmology, 2012. 60(5): p. 428-431.

7. Jiang, X., L. Yang, and Y. Luo, Animal Models of Diabetic Retinopathy. Current Eye Research, 2015. 40(8): p. 761-771.

8. $\quad$ Rakoczy, E.P., et al., Characterization of a mouse model of hyperglycemia and retinal neovascularization. Am J Pathol, 2010. 177(5): p. 2659-70.

9. $\quad$ Behl, Y., et al., Diabetes-Enhanced Tumor Necrosis Factor- $\alpha$ Production Promotes Apoptosis and the Loss of Retinal Microvascular Cells in Type 1 and Type 2 Models of Diabetic Retinopathy. The American Journal of Pathology, 2008. 172(5): p. 1411-1418.

10. Griffin, K.A., et al., Dynamic blood pressure load and nephropathy in the ZSF1 (\&lt;em\&gt;fa\&lt;/em\&gt;/\&lt;em\&gt;fa\&lt;/em\&gt;\&lt;sup\&gt;cp\&lt;/sup\&gt;) model of type 2 diabetes. American Journal of Physiology - Renal Physiology, 2007. 293(5): p. F1605.

11. Dominguez, J.H., et al., Renal injury: Similarities and differences in male and female rats with the metabolic syndrome. Kidney International, 2006. 69(11): p. 1969-1976.

12. Sasongko, M.B., et al., Retinal Vessel Tortuosity and Its Relation to Traditional and Novel Vascular Risk Markers in Persons with Diabetes. Curr Eye Res, 2016. 41(4): p. 551-7.

13. Arboleda-Velasquez, J.F., et al., From pathobiology to the targeting of pericytes for the treatment of diabetic retinopathy. Curr Diab Rep, 2015. 15(2): p. 573.

14. Hammes, H.P., et al., Pericytes and the pathogenesis of diabetic retinopathy. Diabetes, 2002. 51(10): p. 3107-12.

15. Korn, C. and Hellmut G. Augustin, Mechanisms of Vessel Pruning and Regression. Developmental Cell, 2015. 34(1): p. 5-17.

16. Ciulla, T.A., A.G. Amador, and B. Zinman, Diabetic Retinopathy and Diabetic Macular Edema. Diabetes Care, 2003. 26(9): p. 2653.

17. Semenza, G.L., Targeting HIF-1 for cancer therapy. Nat Rev Cancer, 2003. 3(10): p. 721-732.

18. Grigsby, J.G., et al., The Role of Microglia in Diabetic Retinopathy. Journal of Ophthalmology, 2014. 2014: p. 15.

19. Madeira, M.H., et al., Contribution of Microglia-Mediated Neuroinflammation to Retinal Degenerative Diseases. Mediators of Inflammation, 2015. 2015: p. 15.

20. Omri, S., et al., Microglia/Macrophages Migrate through Retinal Epithelium Barrier by a Transcellular Route in Diabetic Retinopathy: Role of PKC $\zeta$ in the Goto Kakizaki Rat Model. The American Journal of Pathology, 2011. 179(2): p. 942-953. 
21. Damani, M.R., et al., Age-related Alterations in the Dynamic Behavior of Microglia. Aging cell, 2011. 10(2): p. 263-276.

22. Nowroozpoor-Dailami, K., et al., Aqueous humor and serum concentrations of soluble MICA and MICB in glaucoma patients. Iran J Immunol, 2014. 11(4): p. 275-81.

23. Baker, R.G., M.S. Hayden, and S. Ghosh, NF-kappaB, inflammation, and metabolic disease. Cell Metab, 2011. 13(1): p. 11-22.

24. Miyamoto, K., et al., Prevention of leukostasis and vascular leakage in streptozotocin-induced diabetic retinopathy via intercellular adhesion molecule-1 inhibition. Proc Natl Acad Sci U S A, 1999. 96(19): p. 10836-41.

25. Tatemoto, K., M. Carlquist, and V. Mutt, Neuropeptide Y--a novel brain peptide with structural similarities to peptide YY and pancreatic polypeptide. Nature, 1982. 296(5858): p. 659-60.

26. Santos-Carvalho, A., A.F. Ambrosio, and C. Cavadas, Neuropeptide Y system in the retina: From localization to function. Prog Retin Eye Res, 2015. 47: p. 19-37.

27. Alvaro, A.R., et al., Neuropeptide Y protects retinal neural cells against cell death induced by ecstasy. Neuroscience, 2008. 152(1): p. 97-105.

28. Santos-Carvalho, A., et al., Neuropeptide Y receptors activation protects rat retinal neural cells against necrotic and apoptotic cell death induced by glutamate. Cell Death Dis, 2013. 4: p. e636.

29. Silva, A.P., et al., Activation of neuropeptide Y receptors is neuroprotective against excitotoxicity in organotypic hippocampal slice cultures. FASEB J, 2003. 17(9): p. 1118-20.

30. Alvaro, A.R., et al., NPY in rat retina is present in neurons, in endothelial cells and also in microglial and Muller cells. Neurochem Int, 2007. 50(5): p. 757-63.

31. Santos-Carvalho, A., et al., Neuropeptide Y receptors Y1 and Y2 are present in neurons and glial cells in rat retinal cells in culture. Invest Ophthalmol Vis Sci, 2013. 54(1): p. 429-43.

32. Jaakkola, U., et al., The Leu7Pro polymorphism of neuropeptide $Y$ is associated with younger age of onset of type 2 diabetes mellitus and increased risk for nephropathy in subjects with diabetic retinopathy. Exp Clin Endocrinol Diabetes, 2006. 114(4): p. 147-52.

33. Koulu, M., et al., Neuropeptide Y and Y2-receptor are involved in development of diabetic retinopathy and retinal neovascularization. Ann Med, 2004. 36(3): p. 232-40.

34. Niskanen, L., et al., Leucine 7 to proline 7 polymorphism in the neuropeptide $y$ gene is associated with retinopathy in type 2 diabetes. Exp Clin Endocrinol Diabetes, 2000. 108(3): p. 235-6.

35. Yoon, H.Z., et al., Neuropeptide Y expression in a mouse model of oxygen-induced retinopathy. Clin Exp Ophthalmol, 2002. 30(6): p. 424-9.

36. Schmid, E., et al., Secretoneurin, substance $P$ and neuropeptide $Y$ in the oxygeninduced retinopathy in C57Bl/6N mice. Peptides, 2012. 37(2): p. 252-7.

37. Wang, X., et al., Expression and regulation of alpha-, beta-, and gammacrystallins in mammalian lens epithelial cells. Invest Ophthalmol Vis Sci, 2004. 45(10): p. 3608-19.

38. Fort, P.E., et al., The retinal proteome in experimental diabetic retinopathy: upregulation of crystallins and reversal by systemic and periocular insulin. Mol Cell Proteomics, 2009. 8(4): p. 767-79. 
39. Reddy, V.S., C.U. Kumar, and G.B. Reddy, Effect of chronic hyperglycemia on crystallin levels in rat lens. Biochem Biophys Res Commun, 2014. 446(2): p. 6027.

40. Reddy, V.S., et al., Hyperglycemia induced expression, phosphorylation, and translocation of alphaB-crystallin in rat skeletal muscle. IUBMB Life, 2015. 67(4): p. 291-9.

41. Horwitz, J., Alpha-crystallin can function as a molecular chaperone. Proc Natl Acad Sci U S A, 1992. 89(21): p. 10449-53.

42. Liedtke, T., et al., Elongation of axons during regeneration involves retinal crystallin beta b2 (crybb2). Mol Cell Proteomics, 2007. 6(5): p. 895-907.

43. Kim, D., et al., TopHat2: accurate alignment of transcriptomes in the presence of insertions, deletions and gene fusions. Genome Biol, 2013. 14(4): p. R36.

44. Chen, W., et al., Increased levels of alphaB-crystallin in vitreous fluid of patients with proliferative diabetic retinopathy and correlation with vascular endothelial growth factor. Clin Exp Ophthalmol, 2017. 45(4): p. 379-384.

45. Kim, Y.H., et al., Reduction of experimental diabetic vascular leakage and pericyte apoptosis in mice by delivery of alphaA-crystallin with a recombinant adenovirus. Diabetologia, 2012. 55(10): p. 2835-2844.

46. Kase, S., et al., alphaB-crystallin regulation of angiogenesis by modulation of $V E G F$. Blood, 2010. 115(16): p. 3398-406.

47. Zhang, C., et al., A potential role for beta- and gamma-crystallins in the vascular remodeling of the eye. Dev Dyn, 2005. 234(1): p. 36-47.

48. Sinha, D., et al., A spontaneous mutation affects programmed cell death during development of the rat eye. Exp Eye Res, 2005. 80(3): p. 323-35.

49. Fischer, D., et al., Crystallins of the beta/gamma-superfamily mimic the effects of lens injury and promote axon regeneration. Mol Cell Neurosci, 2008. 37(3): p. 471-9.

50. Barouch, F.C., et al., Integrin-mediated neutrophil adhesion and retinal leukostasis in diabetes. Invest Ophthalmol Vis Sci, 2000. 41(5): p. 1153-8.

51. Penfold, P.L., et al., Modulation of permeability and adhesion molecule expression by human choroidal endothelial cells. Invest Ophthalmol Vis Sci, 2002. 43(9): p. 3125-30.

52. Scott, A., M.B. Powner, and M. Fruttiger, Quantification of vascular tortuosity as an early outcome measure in oxygen induced retinopathy (OIR). Exp Eye Res, 2014. 120: p. 55-60.

53. Sawamiphak, S., M. Ritter, and A. Acker-Palmer, Preparation of retinal explant cultures to study ex vivo tip endothelial cell responses. Nat Protoc, 2010. 5(10): p. 1659-65.

54. Carpentier, G. Contribution: Angiogenesis Analyzer. 2012 [cited 20125 October 2012]; Available from: http://image.bio.methods.free.fr/ImageJ/?AngiogenesisAnalyzer-for-ImageJ\&lang=en.

55. Van Hove, I., et al., MMP-3 Deficiency Alleviates Endotoxin-Induced Acute Inflammation in the Posterior Eye Segment. Int J Mol Sci, 2016. 17(11).

56. Derks, K.W., et al., Deciphering the RNA landscape by RNAome sequencing. RNA Biol, 2015. 12(1): p. 30-42. 


\section{Supplementary information}
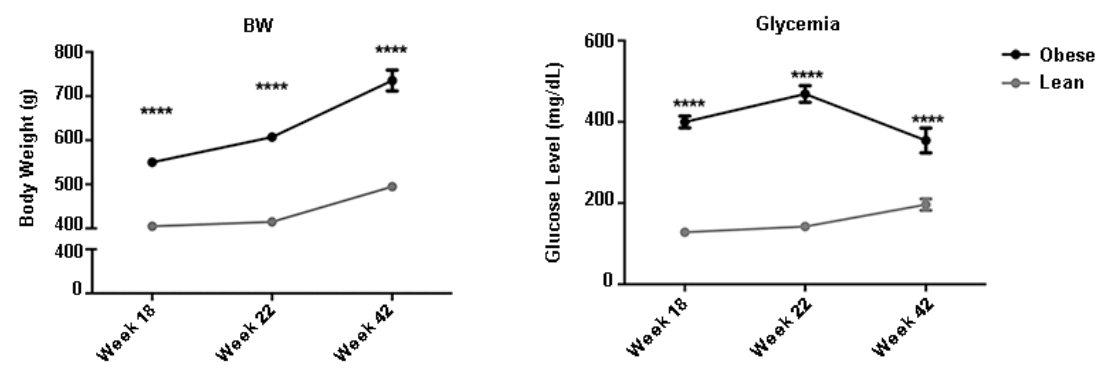

\section{Supplementary Figure 1.}

Body weight and glucose level measured in lean and obese ZSF1 rats at 18,22 and 42 weeks. All values are mean+- SEM, ****p $<0.05$.
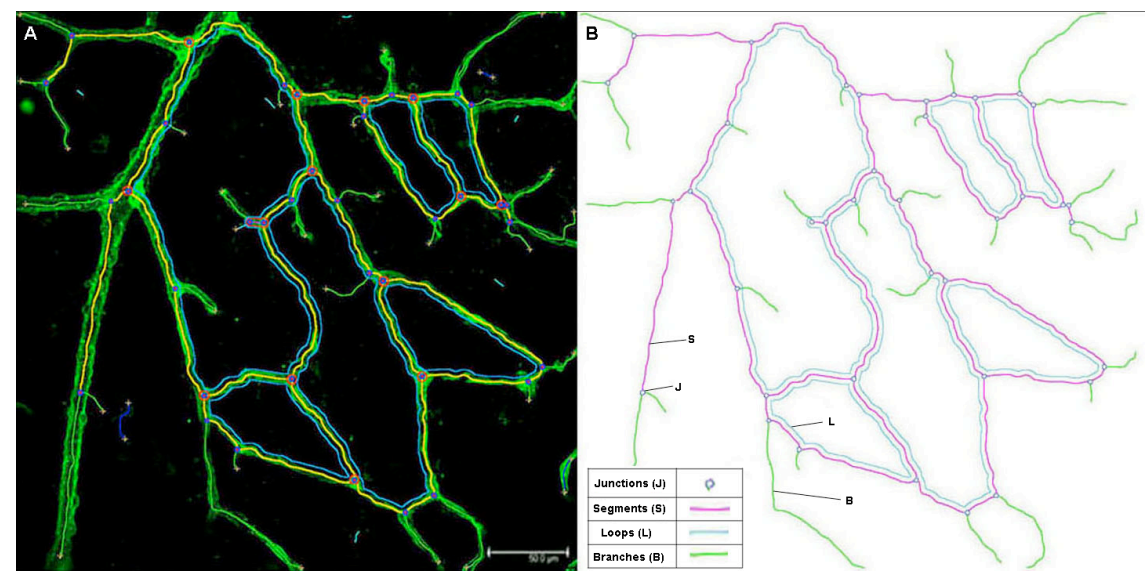

\section{Supplementary Figure 2.}

Example of vascular network analyzed by Angiogenesis Analyzer (A) and map (B) including junctions, segments, loops and branches. Scale bar $=50 \mu \mathrm{m}$. Junctions $(\mathrm{J})$ are indicated as dark blue circles and are the meeting point of several segments. Segments (S) are indicated in fuchsia and are vessels between two junctions. Loops (L) are indicated in light blue and are structures enclosed by segments. Branches (B) are vessels on segments that are not connected on one end. 


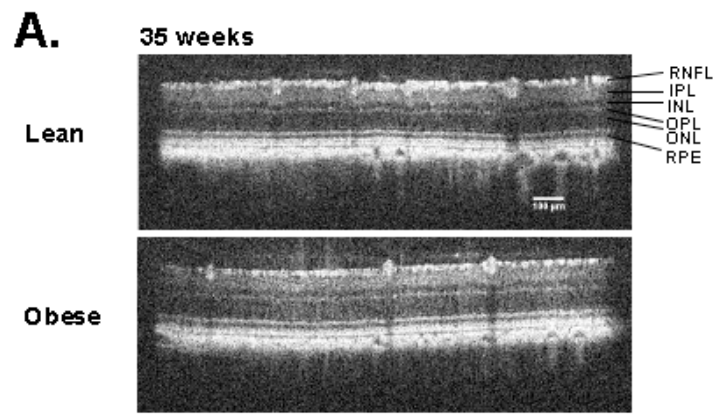

B.

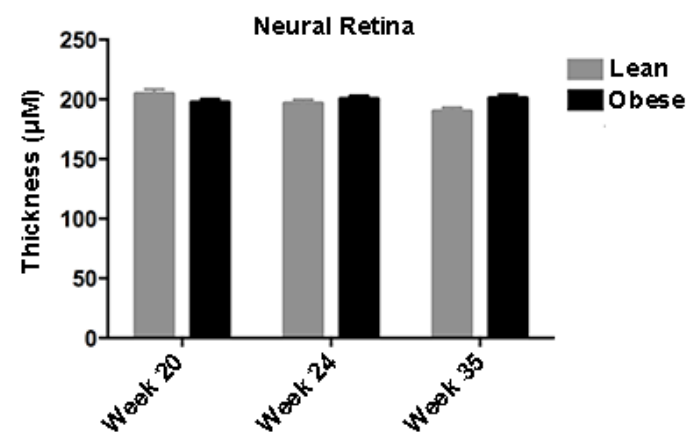

\section{Supplementary Figure 3.}

Spectral domain optical coherence tomography (SD-OCT) analysis performed in lean and obese ZSF1 rats. (A) Representative SD-OCT pictures show no difference in morphology of the neural retina in Lean and Obese rats 35 weeks old. RNFL, retinal nerve fiber layer; IPL, inner plexiform layer; INL, inner nuclear layer; OPL, outer plexiform layer; ONL, outer nuclear layer; RPE, retinal pigmented epithelium. Scale bar $=100 \mu \mathrm{m}$. (B) Analysis of SD-OCT images revealed a similar thickness of the neural retina in Lean and Obese rats at 20,24 and 35 weeks of age, respectively.

Table 1 Lean vs Obese 6 week old

\begin{tabular}{lcc} 
Top canonical pathways & & \\
Name & p-value & Overlap \\
\hline Allograft Rejection Signaling & $\mathbf{3 . 6 4 E - 0 8}$ & $\mathbf{2 4 . 5} \% 12 / 4.9$ \\
OX40 Signaling Pathway & $\mathbf{5 . 9 0 E - 0 8}$ & $\mathbf{2 1 . 3} \% 13 / 61$ \\
Cdc42 Signaling & $\mathbf{8 . 1 4 E - 0 7}$ & $\mathbf{1 3 . 3 \%} 17 / 128$ \\
Antigen Presentation Pathway & $\mathbf{7 . 0 6 E - 0 5}$ & $\mathbf{2 6 . 1} \% 6 / 23$ \\
EIF2 Signaling & $\mathbf{9 . 8 0 E - 0 5}$ & $\mathbf{9 . 0 \%} 18 / 201$
\end{tabular}

\section{Supplementary Table 1.}

Top canonical pathways significantly enriched by Ingenuity Pathway Analysis (IPA) from the differentially expressed genes found between Lean vs Obese 6 weeks old. 
Table 2 Lean vs Obese 42 week old

\begin{tabular}{lcc} 
Top canonical pathways & & \\
Name & p-value & Overlap \\
\hline Antigen Presentation Pathway & $\mathbf{8 . 6 7 E - 0 9}$ & $\mathbf{5 6 , 5 \%} 13 / 23$ \\
OX40 Signaling Pathway & $\mathbf{2 . 2 3 E - 0 8}$ & $\mathbf{3 4 . 4 \%} 21 / 61$ \\
Allograft Rejection Signaling & $\mathbf{7 . 1 4 E}-\mathbf{0 8}$ & $\mathbf{3 6 . 7} \% 18 / 49$ \\
Complement System & $\mathbf{1 . 7 7 E - 0 5}$ & $\mathbf{3 5 , 3} \% 12 / 34$ \\
Cdc42 Signaling & $\mathbf{4 . 1 2 E - 0 5}$ & $\mathbf{2 0 . 0 3 \%} 26 / 128$
\end{tabular}

Supplementary Table 2.

IPA revealed the top 5 canonical pathways expressed in retina isolated from Lean vs Obese 42 weeks old.

Table 3 Lean 6 vs Lean 42 week old

\begin{tabular}{lll} 
Top canonical pathways & & \\
Name & p-value & Overlap \\
\hline EIF2 Signaling & $1.24 \mathrm{E}-06$ & $\mathbf{6 , 5} \% 13 / 201$ \\
Calcium Transport I & $2.05 \mathrm{E}-04$ & $30 \% 3 / 10$ \\
B Cell Development & $2.28 \mathrm{E}-04$ & $16 \% 4 / 25$ \\
Hepatic Fibrosis / Hepatic Stellate Cell Activation & $\mathbf{1 . 4 1 E - 0 3}$ & $\mathbf{4 . 6 \% 8 / 1 7 4}$ \\
nNOS Signaling in Skeletal Muscle Cells & $\mathbf{1 . 0 7 E - 0 2}$ & $\mathbf{1 5 . 4 \% 2 / 1 3}$
\end{tabular}

Supplementary Table 3.

IPA revealed the top 5 canonical pathways expressed in retina isolated from Lean 6 vs Lean 42 weeks old.

Table 4 Obese 6 vs Obese $\mathbf{4 2}$ week old

\begin{tabular}{lcc} 
Top canonical pathways & & \\
Name & p-value & Overlap \\
\hline Leptin Signaling in Obesity & $\mathbf{4 . 4 4 E}-\mathbf{0 3}$ & $\mathbf{3 . 6} \% 3 / 84$ \\
Coagulation System & $\mathbf{7 . 9 1 E - 0 3 ~}$ & $\mathbf{5 . 9} \% 2 / 34$ \\
cAMP-mediated signaling & $\mathbf{1 . 0 6 E - 0 2}$ & $\mathbf{1 . 8 \%} 4 / 214$ \\
Thyronamine and lodothyronamine Metabolism & $\mathbf{1 . 1 8 E - 0 2}$ & $\mathbf{3 3 . 3} \% 1 / 3$ \\
Thyroid Hormone Metabolism I (via Deiodination) & $\mathbf{1 . 1 8 E - 0 2}$ & $\mathbf{3 3 . 3 \%} 1 / 3$
\end{tabular}

Supplementary Table 4.

IPA revealed the top 5 canonical pathways expressed in retina isolated from Obese 6 vs Obese 42 weeks old. 
Chapter 3: MicroRNA expression profile in diabetic patients and Akimba mice

Quentin ROBLAIN, Vincenza CAOLO, Julie LECOMTE, Stephane HEYMANS 


\begin{abstract}
Diabetic retinopathy (DR) is a leading cause of blindness worldwide. In diabetic patients, hyperglycemia can trigger major neurovascular complications within the eye, therefore resulting in pathological neovascularization and inflammation. Intravitreal injection of anti-VEGF is currently used in the clinic for proliferative DR, but it only shows partial efficiency. MicroRNAs are short non-coding RNAs that repress the expression of their target genes. These molecules are involved in a plethora of pathophysiological processes, including many ocular diseases. In the present study, we investigated the expression profile of a series of angiogenesis and inflammation related microRNAs by qRTPCR in the vitreous humors of diabetic patients, and in the retinas isolated from Akimba mice. First, we discussed the microRNA expression profile from vitreous humor samples of diabetic patients and compared it to existing literature. In a second time, we performed the same comparison with the microRNA expression profile from Akimba mice. This strategy led us to identify specific microRNA expression patterns. Overlap of human and mouse results highlighted 5 promising microRNAs for future investigations: miR-142-3p, miR146b-5p, miR-152-3p, miR-199a-3p and miR-320a. A characterization of the potential functional role of these microRNAs in angiogenesis and inflammation during DR will eventually lead to promising target for new therapeutics.
\end{abstract}




\section{Introduction}

Diabetes Mellitus (DM) is one of the world's fastest growing chronic disease, affecting 463 million individuals in 2019 and excepted to affect 578 million by 2030 [1]. Diabetes can lead to complications in many organs and systems such as the heart, the kidney and the nervous system, increasing the overall risk of premature death [2]. Diabetic retinopathy (DR) is a major complication of diabetes that affects the eye and leads to vision impairment and loss. DR affects $35,4 \%$ of diabetic patients and is the fifth leading cause of blindness worldwide [3]. With increasing prevalence of diabetes over years, DR incidence is expected to increase.

DR is a neurovascular pathology triggered by hyperglycemia, affecting different celltype of the retina and involving several biochemical modifications [4]. It can be classified as non-proliferative diabetic retinopathy (NPDR), proliferative diabetic retinopathy (PDR) and macular edema (ME). Specifically, PDR affects $7,2 \%$ of diabetic patients and is characterized by retinal neovascularization and inflammation, due to microglia activation, among other inflammatory cells $[3,5,6]$. Different animal models of DR are available, such as the streptozotocin (STZ)-induced models, the $d b / d b$ mice (spontaneous mutation in the leptin receptor, leading to abnormal phenotypes in metabolic, reproductive and immune systems) and the Akimba mouse model. This latest model exhibits VEGF overexpression together with diabetic background, being thus a model of proliferative DR. However, all these models have their limitations [7, 8]. More recently, it was established that the ZSF1 rat model, despite showing precursor signs of DR, was protected from further progression of the disease, making thus this model a tool for studying resistance to DR development [9]. Anti-VEGF (Vascular Endothelium Growth Factor) therapy has emerged as a potential therapy for the treatment of PDR, overtaking classical laser photocoagulation [10]. In a randomized clinical trial, $46 \%$ of patients administrated with ranibizumab, a VEGF inhibitor, experienced improved vision [11]. However, despite showing a higher efficiency than laser therapy, antiVEGF therapy is not able to restore vision in DR patients. Therefore, the development of new therapeutic tools is urgently needed.

Since their discovery in 1993 in C.elegans [12], microRNAs (also called miRNAs or miRs) have gain a large interest by the scientific community. Several studies have already focused on the potential role of microRNAs as biomarkers or targets for DR $[13,14]$. Most of these studies considered serum or plasma circulating microRNAs, only a few investigated vitreous humors microRNAs. Unfortunately, the overlap of highlighted microRNAs in these studies remains poor. In addition, only a few studies addressed the question of a functional role of a specific microRNA in DR progression by modulating its expression in vivo.

In the present study, we determined an expression profile pattern of angiogenic and inflammatory microRNAs in the vitreous of diabetic patients and compared it to previous similar studies. We also determined for the first time the expression profile of these angiogenic and inflammatory microRNAs in the Akimba mouse, a model of advanced proliferative DR. We highlighted 5 specific microRNAs whose expression were dysregulated 
both in the Akimba mouse and in the vitreous humor of diabetic patients. Thus, we have identified a set of microRNAs that may have a regulatory function in DR.

\section{Material and Methods}

\section{- Human vitreous humors}

The vitreous humor is a clear, colorless fluid that fills the space between the lens and the retina. It is made of $99 \%$ of water, the rest being mainly type II and type IX collagen fibers, hyaluronic acid, soluble proteins and hyalocytes. The vitreous helps to hold the spherical shape of the eye and helps to keep the retina in the posterior part of the eye. For a number of issues such as retinal detachment or vitreous hemorrhage, ophthalmologists can proceed to the removal of a part of the vitreous during a procedure called a vitrectomy.

Vitreous humors from healthy control (i.e. non-diabetic) and diabetic patients were collected during vitrectomy needed for a medical purpose. After collection, fresh and undiluted samples were centrifuged to eliminate any circulating cells and then kept at -80 degrees until RNA isolation. Nine vitreous humors from healthy control patients as well as 4 vitreous humors from diabetic patients were collected during a 3-year period of time.

\section{- Akimba mouse model}

The Akimba mouse model was generated and described by E.P. Rakoczy in 2010 [8]. This model was established to study the interaction of 2 main characteristics of diabetic retinopathy: hyperglycemia and VEGF overexpression. The Akimba mouse model was generated by crossing the Akita mouse with the Kimba mouse. Non-obese type-1 diabetic Akita mouse (also known as Mody mouse) exhibits a spontaneous mutation in the Insulin 2 gene, leading to severe pancreatic $\beta$-cells dysfunction [15]. Transgenic Kimba mouse exhibits transient human $\mathrm{VEGF}_{165}$ photoreceptor-specific overexpression, leading to retinal neovascularization [16]. Akimba model retains both parental phenotypes, type-1 diabetic background and retinal neovascularization, being thus a suitable and attractive model for studying proliferative diabetic retinopathy.

Akita, Kimba, Akimba and relative C57B16 Wild-Type (WT) mice were kindly provided by Oxurion. Three Akita mice, along with 5 Kimba, 5 Akimba and 5 WT mice were included in this study. Mice were scarified 3 months after birth, then eyes were enucleated and retinas of OD and OS were isolated and pooled together for each individual. Pooled retinas were then snap frozen in liquid nitrogen and kept at - 80 degrees until RNA isolation.

\section{- $\quad$ RNA extraction, Reverse transcription and qRT-PCR}

Vitreous humors were lysed in lysis buffer by vortexing, using the same quantity of vitreous humor $(200 \mu \mathrm{L})$ for each patient. RNA was isolated from the lysate with miRNeasy serum/plasma kit (Qiagen) according to the manufacturer's protocol. Reverse transcription was performed with Universal cDNA synthesis kit (Exiqon) according to the manufacturer's instructions. qRT-PCR was performed with iQ SYBR green Supermix (Bio-Rad) according to the manufacturer's instructions. MiRNA levels were assessed with miRCURY LNA 
miRNA PCR Assay (Qiagen). All data were normalized to C. elegans miR-39 (Qiagen) expression as a spike-in control added to all samples prior to RNA isolation.

Retinas were lysed with stainless steel beads (Qiagen) in lysis buffer with the TissueLyser LT (Qiagen). RNA was isolated from the lysate with mirVANA kit (Ambion) according to the manufacturer's instructions. Reverse transcription was performed with miScript RT Kit II (Qiagen) using the same RNA input according to the manufacturer's instructions. qRT-PCR was performed with iQ SYBR Green Supermix (Bio-Rad) according to the manufacturer's protocol, with $10 \mathrm{ng}$ cDNA as input. MiRNA levels were assessed with miRCURY LNA miRNA PCR Assay (Qiagen). All data were normalized to GAPDH and HPRT expression as housekeeping genes.

\section{- Selection of candidate microRNAs}

The 32/30 microRNAs tested by qRT-PCR in human/mouse samples were chosen from a literature research. This literature research was carried out in early 2017 on PubMed database and focused on determining a list of microRNAs with potential or known functions in angiogenic processes as well as in inflammatory processes. This biased approach for the selection of candidate microRNAs was intentionally used to highlight potential microRNAs implicated in inflammation and/or angiogenesis, the main biological features of proliferative diabetic retinopathy.

\section{- Data analysis}

All data underwent a statistical analysis using Prims7 software. All graphs are presented as mean +/- SEM. Mann-Whitney test or One-way ANOVA followed by post-hoc multiple comparisons tests were used to determine statistical significance. Statistical significance was defined as $\mathrm{p} \leq 0.05$. 


\section{Results}

\section{MicroRNA expression profile in the vitreous of diabetic patients}

In this study, we first analyzed the expression of 32 pre-selected microRNAs in the vitreous of diabetic patients by qRT-PCR (Fig. 1). Among these, 10 had a significant increased expression in diabetic patients when compared to healthy controls: miR-21-5p, miR-23b-3p, miR-29b-3p, miR-142-3p, miR-142-5p, miR-146b-5p, miR-152-5p, miR199a-3p, miR-320a and miR-378a-3p.

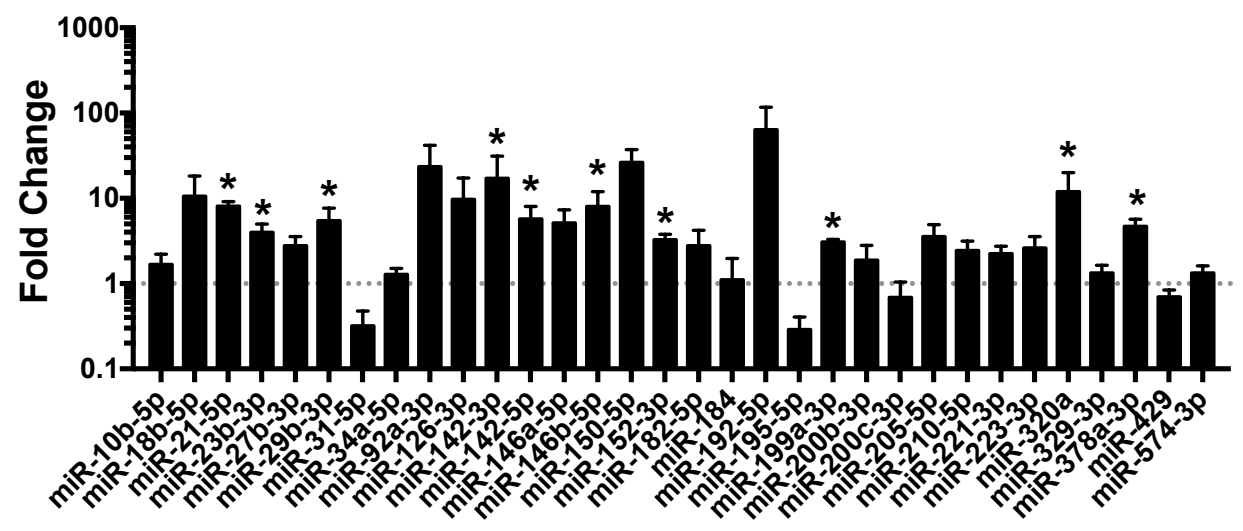

Figure 1. MicroRNA expression profile in the vitreous of diabetic patients

MicroRNA expression profile in the vitreous of diabetic patients compared to healthy vitreous. Diabetic vitreous results are presented as fold change to healthy vitreous (not shown on the graph). Results are presented as mean +SEM. Mann Whitney test $(\mathrm{n}=3-9$ per experimental group) $(*=\mathrm{p}<0.05)$. 


\section{MicroRNA expression profile in the retinas of Akimba and related parental mouse models}

We next analyzed the expression of the same 32 microRNAs in the retinas of Akita, Kimba and Akimba mice. MiR-329-3p and 429 were not detected in our samples. Out of the remaining 30 microRNAs whose expression was detected, 3 were dysregulated in Akita mice, 13 in Kimba mice and 20 in Akimba mice (Tab. 1). Akita mice exhibited increased expression of miR-21-5p and 221-3p, and decreased expression of miR-378a-3p. Kimba mice exhibited increased expression of miR-34a-5p, 142-3p, 146a-5p, 146b-5p, 195-5p and 223-3p, while expressions of miR-18b-5p, 21-5p, 27b-3p, 92a-3p, 150-5p, 182-5p and 192$5 \mathrm{p}$ were decreased. Akimba mice exhibited increased expression of miR-10b-5p, 31-5p, 34a5p, 126-3p, 142-3p, 146a-5p, 146b-5p, 152-3p, 184, 195-5p, 199a-3p, 210-5p, 221-3p, $223-$ $3 \mathrm{p}, 320 \mathrm{a}$ and $574-3 \mathrm{p}$, while expressions of miR-92a-3p, 150-5p, 182-5p and 192-5p were decreased (Fig. 2).

\begin{tabular}{|c|c|c|c|}
\hline & Akita & Kimba & Akimba \\
\hline miR-10b-5p & 0 & 0 & + \\
\hline miR-18b-5p & 0 & - & 0 \\
\hline miR-21-5p & + & - & 0 \\
\hline miR-23b-3p & 0 & 0 & 0 \\
\hline miR-27b-3p & 0 & - & 0 \\
\hline miR-29b-3p & 0 & 0 & 0 \\
\hline miR-31-5p & 0 & 0 & + \\
\hline miR-34a-5p & 0 & + & + \\
\hline miR-92a-3p & 0 & - & - \\
\hline miR-126-3p & 0 & 0 & + \\
\hline miR-142-3p & 0 & 0 & + \\
\hline miR-142-5p & 0 & + & 0 \\
\hline miR-146a-5p & 0 & + & + \\
\hline miR-146b-5p & 0 & + & + \\
\hline miR-150-5p & 0 & - & - \\
\hline miR-152-3p & 0 & 0 & + \\
\hline miR-182-5p & 0 & - & - \\
\hline miR-184 & 0 & 0 & + \\
\hline miR-192-5p & 0 & - & - \\
\hline miR-195-5p & 0 & + & + \\
\hline miR-199a-3p & 0 & 0 & + \\
\hline miR-200b-3p & 0 & 0 & 0 \\
\hline miR-200c-3p & 0 & 0 & 0 \\
\hline miR-205-5p & 0 & 0 & 0 \\
\hline miR-210-5p & 0 & 0 & + \\
\hline miR-221-3p & + & 0 & + \\
\hline miR-223-3p & 0 & + & + \\
\hline miR-320a & 0 & 0 & + \\
\hline miR-378a-3p & - & 0 & 0 \\
\hline miR-574-3p & 0 & 0 & + \\
\hline
\end{tabular}

Table 1. Overview of microRNA dysregulation in the retinas of Akita, Kimba and Akimba mice model

0 = no significant difference compared to WT mice; + = upregulated compared to WT mice; - = downregulated compared to WT mice. 


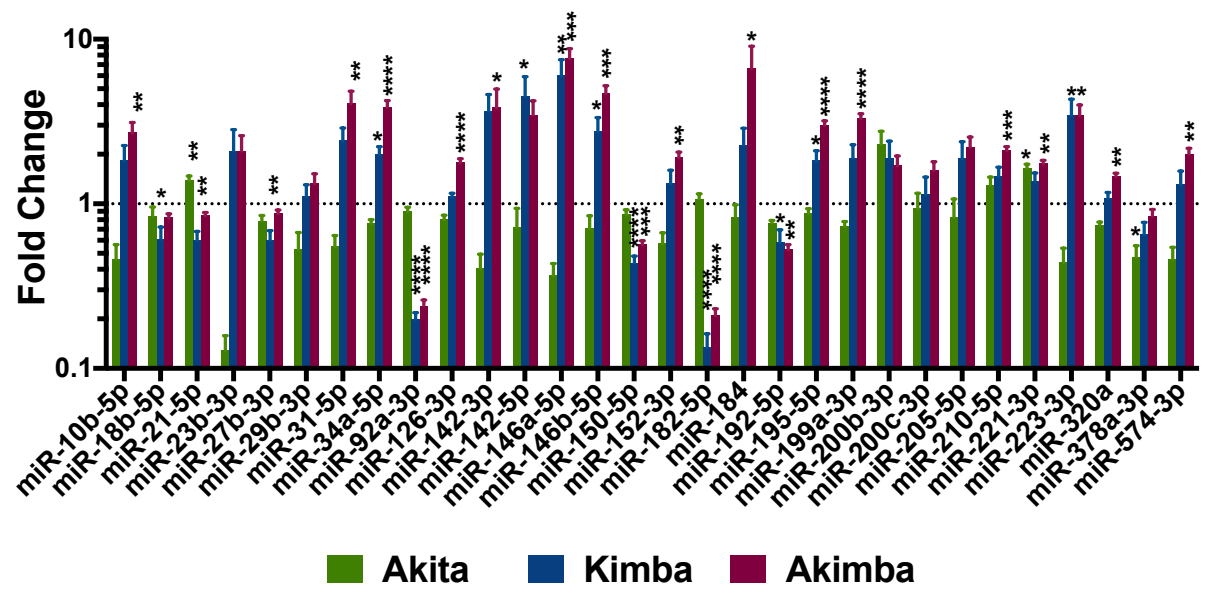

Figure 2. MicroRNA expression profile in the retinas of Akita, Kimba and Akimba mice model

MicroRNA expression profile in the retinas of Akita, Kimba and Akimba mice compared to WT mice. Akita, Kimba and Akimba results are presented as fold change to WT mice (not shown on the graph). Results are presented by mean + - SEM. One-way ANOVA + multiple comparison test $(n=3-5$ per experimental group) $(*=\mathrm{p} \leq 0.05 ; * *=\mathrm{p} \leq 0.01 ; * * *=\mathrm{p} \leq 0.001 ; * * * *=\mathrm{p} \leq 0.0001)$. 


\section{Overlap of overexpressed microRNAs in diabetic patients and Akimba mice}

The overlap of upregulated microRNAs in both human and mouse samples highlighted 5 microRNAs whose expression was increased compared to control conditions. These microRNAs were miR-142-3p, miR-146b-5p, miR-152-3p, miR-199a-3p and miR-320a (Fig. 3).

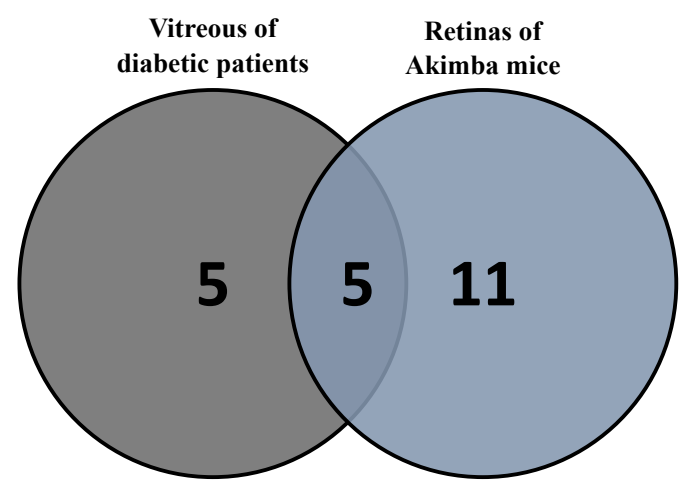

Figure 3. Venn diagram showing overlap of upregulated microRNAs in the vitreous of diabetic patients and the retinas of Akimba mice

Among the 32 microRNAs tested in the vitreous of diabetic patients and the 30 microRNAs tested in the retinas of Akimba mice, 5 microRNAs showed a common overexpression in both models. These microRNAs are miR142-3p, miR-146b-5p, miR-152-3p, miR-199a-3p and miR-320a. 


\section{Discussion}

\section{Vitreous humors of diabetic patients exhibit a specific microRNA expression profile}

In this study, we analyzed the expression profile of a set of microRNAs in the vitreous of diabetic patients. Human samples were initially collected in the context of a research not focusing on DR. Thus, we were only able to collect vitreous of diabetic patients and healthy control patients, but no vitreous of NPDR or PDR patients had been collected. Several studies have recently reported microRNAs expression profile in the vitreous of diabetic patients with or without diabetic retinopathy, all recently reviewed in [13] and [14]. Interestingly, our results show similarities with these previous studies but also major differences.

MiR-21, previously described as a pro-angiogenic and pro-fibrotic microRNA, was upregulated in our samples of diabetic patients, in line with its vitreous expression in patients with a proliferative vitreoretinal disease patients [17]. Interestingly, miR-21 was also upregulated in the serum of PDR patients when compared to NPDR patients, making this microRNA a monitoring factor for detecting the progression of PDR from NPDR [18]. Thus, miR-21 appears to be a microRNA implicated in both primary onset and DR progression. Future studies will have to determine if a correlation between miR-21 quantity and DR progression can be observed. In a previous study, miR-29a and miR-320a were upregulated in PDR vitreous when compared to control (macular hole, MH) [19]. In the same study, miR320a was also upregulated in the serum of PDR patients, but not miR-29a. Similarly, our analysis revealed a similar increase of miR-320a and miR-29b, a close isomiR to miR-29a, in the vitreous of diabetic patients. While miR-142-3p was specifically expressed in the vitreous of PDR patients but not detected in the vitreous of MH control patients [19], we detected miR-142-3p in both diabetic and control group, with a significantly higher expression in the diabetic group. In the same study, miR-142-5p was not detected in either control (macular hole) patients or PDR patients. Similarly, miR-152-5p was upregulated in our vitreous samples of diabetic patients, but was not in the other studies including both $\mathrm{MH}$ and PDR groups.

In conclusion, our set of diabetic vitreous samples shows partial matches with what is known from the literature. However, we also highlighted specific features of miRNA expression that was not describe until now. In this way, we showed a specific overexpression of miR-23b-3p, miR-146b-5p, miR-152-5p and miR-378a-3p. To our knowledge, these microRNAs have never been described yet in a context of diabetic vitreous samples. Despite showing particular specificities, our set of samples failed to highlight some well-described microRNAs in DR such as miR-200b [20, 21].

Taken together our data suggest that the vitreous microRNA content of diabetic patients partially overlap the vitreous content of DR patients. However, all these data have to be considered with care due to methodological considerations and limitations. First, the procedure of vitreous collection is not always described, particularly the centrifugation step. Indeed, vitreous humor can content several circulating cell-types such as macrophages and red blood cells, in particular in hemorrhagic and inflammatory conditions. Thus, 
centrifugation allows the elimination of cell pellets. The resulting microRNA expression profile that we obtained in this study is thus originating from exosomes or microvesicles, which are known to be carrier of microRNAs in different body fluid such as vitreous humor $[22,23]$. Second, the method of detection used has to be taken in consideration. In most studies, microRNA expression profile was first obtained by microarray analysis than confirmed by qRT-PCR. Here, we assessed the microRNA composition by qRT-PCR, leading to possible biased results. Moreover, which one of the mature microRNA strand (5p or $3 p$ ) was used for a particular study is not always described by the authors. Finally, the type of control patients might interfere with the comparison of the different studies. Indeed, several clinical feature of control patients -sex, age and co-morbidities among other- could alter the expression profile.

\section{Retinas of Akimba mice exhibit a specific microRNA expression profile}

Out of the 32 microRNAs tested in human samples, only 30 were expressed in mouse samples: miR-329-3p and miR-429 were not detected in Akimba mice and parental strains. To our knowledge, this is the first time that a microRNAs expression profile is reported in the Akimba mouse model of PDR.

Akimba mice show enhanced pro-inflammatory and pro-angiogenic characteristics such as activation of NLRP3 inflammasome and loss of blood-retinal barrier (BRB) [24, 25]. Thus, a large microRNA dysregulation in Akimba retinas was to be expected. Two of the most upregulated microRNAs were miR-146a-5p and miR-146b-5p, well-described for their regulatory functions in immune responses and angiogenesis [26]. These distinct microRNAs only differ by 2 nucleotides in the 3' region and share identical seed sequence, and therefore share predicted downstream targets. Their overexpression is thus concomitant with the highly inflammatory environment encountered in Akimba retinas. At the functional level, intravitreal delivery of miR-146 successfully attenuates inflammatory phenotype in both SZT-induced diabetic rat model as well as in a model of Experimental Autoimmune Anterior Uveitis (EAAU), another common ocular inflammation disorder [27, 28]. Taken together, these data suggest a potential implication of both miR-146a-5p and miR-146b-5p in PDR. This should be further confirmed by intravitreal injection of miR-146 inhibitor and mimic in the Akimba model.

In parallel to the 2 most upregulated microRNAs, the expression of miR-92a-3p and miR-182-5p is strongly decreased in the eyes of Akimba mice. MiR-92a-3p is a proangiogenic mediator produced by endothelial cells (EC) and packed into extracellular vesicles. In a diabetic context, miR-92a-3p is elevated in high-density lipoprotein (HDP) fraction in reconstituted HDL-infused mice early after ischemia [29]. However, no description of this microRNA has been made in a DR context. It is thus surprising to observe that miR-92a-3p is downregulated in Kimba and Akimba mice while they show a strong neovascularization phenotype. This might be explained by the fact that miR-92a-3p is transcribed from the miR-17/92 cluster [30]. Further characterization of miR-92a-3p expression in Akimba samples should thus be accompanied by assessment of expression level of other microRNAs originating from the same cluster such as miR-17 or miR-20a. The 
second most downregulated microRNA was miR-182-5p, with poorly described function. MiR-182 is overexpressed in diabetic nephropathy (DN) patients and is responsible for podocytes apoptosis, leading to an increased risk of DN [31]. It is also a key regulator of diabetic corneal nerve regeneration [32]. Implications of miR-182-5p in the diabetic eye might thus be related to apoptosis and regeneration, but requires further investigations.

While Akimba mice retain both VEGF overexpression and diabetic status from parental strains, Akita strain only have a DM background. Thus, comparison of microRNAs dysregulation in diabetic patients and diabetic mice could be relevant. Only a poor overlap in microRNAs expression profile in Akita's retinas and vitreous of diabetic patients is present. While miR-21-5p is overexpressed in both human and Akita mouse samples, miR$378 \mathrm{a}-3 \mathrm{p}$ is downregulated in human and upregulated in mouse. MiR-221-3p overexpression is only observed in mouse and not in human. Even if miR-221-3p was not dysregulated in our set of human samples, this specific microRNA is overexpressed in the serum of PDR patients [33]. A positive correlation between miR-221 level and DR progression was observed. Thus, miR-221 concentration seems to increase both in NPDR and PDR compared to healthy patients.

Despite showing strong inflammatory and angiogenic phenotypes, the Akimba mouse model may not be the most appropriate model for proliferative DR since it also shows severe accessibility limitations. Indeed, this mouse model is protected under a patent and its accessibility for academic research is very complicated. Moreover, breeding of Akimba mice is difficult, since crossing of parental strains results in a low number of viable pups (+- $10 \%$ of viable pups). Altogether, these particularities make the Akimba mouse model poorly accessible.

\section{Promising targets for further proliferative DR investigations}

In order to define a specific set of angiogenic and/or inflammatory related microRNAs in PDNR, we merged the data in diabetic patients and Akimba mice. As such, miR-142-3p, miR-146b-5p, miR-152-3p, miR-199a-3p and miR-320a emerge as overlapping targets, and future studies will have to address their functional role in DR progression. To our knowledge, no studies have currently addressed the question of a functional role of these microRNAs in a PDR context. Local or systemic delivery of miR inhibitor and mimic in the Akimba model and parental strains as well as in other animal models of DR could therefore improve our knowledge about the biological role of these microRNAs. 


\section{Conclusion}

Since their discovery, microRNAs appeared to be master regulators of numerous physiological and pathological processes. In the course of proliferative diabetic retinopathy, a vision-threatening ocular disease, many microRNAs show dysregulated expression profile. Among these microRNAs, some are of particular interest since their dysregulation is concomitantly observed in both animal models and human samples. Thus, further characterization of microRNAs expression profile could help in the diagnosis of PDR. Here, we investigated the expression profile of a large panel of microRNAs expressed in the vitreous of diabetic patients and in the retinas of Akimba mice. We also defined a set of 5 specific microRNAs, namely miR-142-3p, miR-146b-5p, miR-152-3p, miR-199a-3p and miR-320a, that may be deeper characterized in the context of PDR.

\section{Acknowledgements}

The authors kindly acknowledge Dr Joachim Van Calster (KU Leuven-UZ Leuven, Belgium) for providing human vitreous humor samples and Oxurion ${ }^{\circledR}$ (a vision science company based in Leuven, Belgium) for providing Akimba, Kimba, Akita and WT retina samples.

\section{Authors contribution}

QR wrote the manuscript. QR, VC and JL performed the experiments. JL collected human samples. SH designed the study. 


\section{References}

1. Saeedi, P., et al., Global and regional diabetes prevalence estimates for 2019 and projections for 2030 and 2045: Results from the International Diabetes

Federation Diabetes Atlas, 9(th) edition. Diabetes Res Clin Pract, 2019. 157: p. 107843.

2. $\quad$ Organization, W.H., Global Report on Diabetes. 2016.

3. Yau, J.W., et al., Global prevalence and major risk factors of diabetic retinopathy. Diabetes Care, 2012. 35(3): p. 556-64.

4. Lopez-Galvez, M.I., F. Manco Lavado, and J.C. Pastor, Diabetic Retinopathy, in Handbook of Nutrition, Diet and the Eye. 2014. p. 41-51.

5. Tang, J. and T.S. Kern, Inflammation in diabetic retinopathy. Prog Retin Eye Res, 2011. 30(5): p. 343-58.

6. Altmann, C. and M.H.H. Schmidt, The Role of Microglia in Diabetic Retinopathy: Inflammation, Microvasculature Defects and Neurodegeneration. Int J Mol Sci, 2018. 19(1).

7. Cai, X. and J.F. McGinnis, Diabetic Retinopathy: Animal Models, Therapies, and Perspectives. J Diabetes Res, 2016. 2016: p. 3789217.

8. Rakoczy, E.P., et al., Characterization of a mouse model of hyperglycemia and retinal neovascularization. Am J Pathol, 2010. 177(5): p. 2659-70.

9. Caolo, V., et al., Resistance to retinopathy development in obese, diabetic and hypertensive ZSF1 rats: an exciting model to identify protective genes. Sci Rep, 2018. 8(1): p. 11922.

10. Bahrami, B., et al., Anti-VEGF Therapy for Diabetic Eye Diseases. Asia Pac J Ophthalmol (Phila), 2017. 6(6): p. 535-545.

11. Cheung, N., I.Y. Wong, and T.Y. Wong, Ocular anti-VEGF therapy for diabetic retinopathy: overview of clinical efficacy and evolving applications. Diabetes Care, 2014. 37(4): p. 900-5.

12. Lee, R.C., R.L. Feinbaum, and V. Ambros, The C. elegans heterochronic gene lin4 encodes small RNAs with antisense complementarity to lin-14. Cell, 1993. 75(5): p. 843-54.

13. Martinez, B. and P.V. Peplow, MicroRNAs as biomarkers of diabetic retinopathy and disease progression. Neural Regen Res, 2019. 14(11): p. 1858-1869.

14. Li, X., et al., MicroRNAs: Potential Targets in Diabetic Retinopathy. Horm Metab Res, 2020. 52(3): p. 142-148.

15. Wang, J., et al., A mutation in the insulin 2 gene induces diabetes with severe pancreatic beta-cell dysfunction in the Mody mouse. J Clin Invest, 1999. 103(1): p. 27-37.

16. Lai, C.M., et al., Generation of transgenic mice with mild and severe retinal neovascularisation. Br J Ophthalmol, 2005. 89(7): p. 911-6.

17. Usui-Ouchi, A., et al., Upregulation of Mir-21 Levels in the Vitreous Humor Is Associated with Development of Proliferative Vitreoretinal Disease. PLoS One, 2016. 11(6): p. e0158043.

18. Qing, S., et al., Serum miRNA biomarkers serve as a fingerprint for proliferative diabetic retinopathy. Cell Physiol Biochem, 2014. 34(5): p. 1733-40.

19. Hirota, K., et al., Comparisons of microRNA expression profiles in vitreous humor between eyes with macular hole and eyes with proliferative diabetic retinopathy. Graefes Arch Clin Exp Ophthalmol, 2015. 253(3): p. 335-42. 
20. Gomaa, A.R., E.T. Elsayed, and R.F. Moftah, MicroRNA-200b Expression in the Vitreous Humor of Patients with Proliferative Diabetic Retinopathy. Ophthalmic Res, 2017. 58(3): p. 168-175.

21. McArthur, K., et al., MicroRNA-200b regulates vascular endothelial growth factor-mediated alterations in diabetic retinopathy. Diabetes, 2011. 60(4): p. 1314-23.

22. Zhang, W., S. Chen, and M.L. Liu, Pathogenic roles of microvesicles in diabetic retinopathy. Acta Pharmacol Sin, 2018. 39(1): p. 1-11.

23. Zhao, Y., et al., Liquid Biopsy of Vitreous Reveals an Abundant Vesicle Population Consistent With the Size and Morphology of Exosomes. Transl Vis Sci Technol, 2018. 7(3): p. 6.

24. Chaurasia, S.S., et al., The NLRP3 Inflammasome May Contribute to Pathologic Neovascularization in the Advanced Stages of Diabetic Retinopathy. Sci Rep, 2018. 8(1): p. 2847.

25. Wisniewska-Kruk, J., et al., Molecular analysis of blood-retinal barrier loss in the Akimba mouse, a model of advanced diabetic retinopathy. Exp Eye Res, 2014. 122: p. 123-31.

26. Testa, U., et al., miR-146 and miR-155: Two Key Modulators of Immune Response and Tumor Development. Noncoding RNA, 2017. 3(3).

27. Zhuang, P., C.K. Muraleedharan, and S. Xu, Intraocular Delivery of miR-146 Inhibits Diabetes-Induced Retinal Functional Defects in Diabetic Rat Model. Invest Ophthalmol Vis Sci, 2017. 58(3): p. 1646-1655.

28. Hsu, Y.R., et al., MicroRNA-146a Alleviates Experimental Autoimmune Anterior Uveitis in the Eyes of Lewis Rats. Mediators Inflamm, 2017. 2017: p. 9601349.

29. Hourigan, S.T., et al., The regulation of miRNAs by reconstituted high-density lipoproteins in diabetes-impaired angiogenesis. Sci Rep, 2018. 8(1): p. 13596.

30. Mogilyansky, E. and I. Rigoutsos, The miR-17/92 cluster: a comprehensive update on its genomics, genetics, functions and increasingly important and numerous roles in health and disease. Cell Death Differ, 2013. 20(12): p. 1603-14.

31. Ming, L., et al., Excessive apoptosis of podocytes caused by dysregulation of microRNA-182-5p and CD2AP confers to an increased risk of diabetic nephropathy. J Cell Biochem, 2019. 120(10): p. 16516-16523.

32. Wang, Y., et al., microRNA-182 Mediates Sirt1-Induced Diabetic Corneal Nerve Regeneration. Diabetes, 2016. 65(7): p. 2020-31.

33. Liu, H.N., et al., Serum microRNA-221 as a biomarker for diabetic retinopathy in patients associated with type 2 diabetes. Int J Ophthalmol, 2018. 11(12): p. 18891894. 


\section{Chapter 4: Intravitreal injection of anti-miRs against miR-142-3p reduces angiogenesis and microglia activation in a mouse model of laser-induced choroidal neovascularization}

Quentin Roblain $^{1,2}$, Thomas Louis ${ }^{1}$, Cassandre Yip ${ }^{1}$, Louis Baudin ${ }^{1}$, Ingrid Struman ${ }^{3}$, Vincenza Caolo $^{5}$, Vincent Lambert ${ }^{1,4}$, Julie Lecomte ${ }^{1,}$ ", Agnès Noël ${ }^{1, ~ *} \&$ Stephane Heymans ${ }^{2,5, *}$

${ }^{1}$ Laboratory of Tumor and Development Biology, GIGA-Cancer, University of Liège, Liège, Belgium.

${ }^{2}$ Department of Cardiology, CARIM School for Cardiovascular Diseases, Faculty of Health, Medicine and Life Sciences, Maastricht University, Maastricht, The Netherlands.

${ }^{3}$ Molecular angiogenesis laboratory, GIGA-Cancer, University of Liège, Liège, Belgium.

${ }^{4}$ Ophthalmic Tissue Bank, Department of Ophthalmology, University Hospital of Liège, Sart-Tilman, Belgium

5 Department of Cardiovascular Sciences, Centre for Molecular and Vascular Biology, KU Leuven, Leuven, Belgium.

* These authors equally contributed to the study

In revisions in Aging 
102 


\begin{abstract}
Age-related macular degeneration (AMD) is a worldwide leading cause of blindness affecting individuals over 50 years old. The most aggressive form, wet AMD, is characterized by choroidal neovascularization $(\mathrm{CNV})$ and inflammation involving microglia recruitment. By using a laser-induced CNV mouse model, we provide evidence for a key role played by miR-142-3p during CNV formation. MiR-142-3p was overexpressed in murine CNV lesions and its pharmacological inhibition decreased vascular and microglia densities by $46 \%$ and $30 \%$, respectively. Consistently, miR-142-3p overexpression with mimics resulted in an increase of $136 \%$ and $126 \%$ of blood vessels and microglia recruitment. Interestingly, miR-142-3p expression was linked to the activation state of mouse microglia cells as determined by morphological analysis (cell solidity) through a computational method. In vitro, miR-142-3p overexpression in human microglia cells (HMC3) modulated microglia activation, as shown by CD68 levels. Interestingly, miR142-3p modulation also regulated the production of VEGF-A, the main pro-angiogenic factor. Together, these data strongly support the unprecedented importance of miR-142-3p-dependent vascularinflammation axis during CNV progression, through microglia activation.
\end{abstract}




\section{Introduction}

Globally, 2.2 billion people suffer of vision impairment or blindness [1]. Age-related macular degeneration (AMD) is the fourth leading cause of blindness worldwide [2], affecting $8.7 \%$ of population. This percentage is expected to increase due to the ageing population [3]. Although less frequent than atrophic (or dry) AMD, neovascular (or wet) AMD represents the most vision-threatening form of the disease [4]. It is mainly characterized by choroidal neovascularization (CNV), the formation of abnormal blood vessels arising from the choroid and invading the subretinal space, leading to vision impairment [5]. This angiogenic process is associated with the recruitment and proliferation of inflammatory cells including neutrophils, macrophages and microglia [6, 7]. The latter cells are the main resident immune cells and contributors to the innate immunity within the central nervous system, including the retina. At the experimental level, rodent models of laser-induced CNV are the most broadly used for neovascular AMD pre-clinical research [7, 8]. They recapitulate the angiogenic and inflammatory phases of the disease including microglia implication. Antibody-based anti-VEGF (Vascular Endothelium Growth Factor) therapy is still the golden standard for the management of neovascular AMD [9]. Unfortunately, around one fifth of neovascular AMD patients do not or poorly respond to anti-VEGF therapy [10]. Therefore, additional therapies are urgently required.

MicroRNAs, also called miRs or miRNAs, are short non-coding RNA species of about 20-24 nucleotides length, which act as post-transcriptional gene-expression regulators. A single mature microRNA can bind to several mRNAs and a single mRNA can be targeted by several miRNAs [11-13]. Many ocular diseases are regulated by short non-coding RNAs, and a growing body of literature related to miRNA complications in eye disorders is currently emerging $[14,15]$. To date, several teams have focused on establishing microRNAs as potential AMD biomarkers in both human and mouse study [16, 17]. However, only a few of these studies addressed the question of a functional role of microRNAs in AMD [18]. Through their capacity to regulate biological processes such as angiogenesis and inflammation [19,20], a functional contribution of miRNA in AMD is expected, but poorly documented. Hence, miRNA as therapeutic target for the treatment of AMD is an emerging concept for future drug design.

In the present study, we searched for microRNAs with functional implication in CNV development. We first determined the expression profile of a set of angiogenesis and inflammation-related microRNAs in mouse CNV lesions. Inhibition and overexpression of miR-142-3p, one of the top dysregulated microRNAs, regulated both vascular and inflammatory phenotypes. Notably, microglia recruitment and activation were enhanced by miR-142-3p mimic and reduced by miR-142-3p inhibitor both in vitro and in vivo. Our work provides evidence that miR-142-3p is a functional mediator during CNV progression and acts as a microglia cell activator. MiR-142-3p is worth considering as a target for future neovascular AMD therapeutics. 


\section{Material and methods}

\section{- Laser-induced choroidal neovascularization mouse model}

$\mathrm{CNV}$ was induced in mice by laser-mediated choroid burn as previously described [7]. Briefly, 6 to 8 weeks old C57B16 mice (all males) (Janvier, France) were anesthetized by intraperitoneal injection of a mixture of ketamine $(50 \mathrm{mg} / \mathrm{kg})$ and medetomidine $(0.5 \mathrm{mg} / \mathrm{kg})$. A few minutes before laser induction, pupils were dilated with Tropicol (Théa Pharma, France). Four laser burns per eye were performed with MicronIV instrument (Phoenix Lab, USA) leading to Bruch's membrane local destruction and the subsequent choroidal neovascularization. Mice were than woken up by intraperitoneal administration of atipamezole (1mg/kg). Mice were sacrificed 5 (D5) or 7 days (D7) after laser induction by cervical dislocation. All in vivo experiments were approved by the local Animal Ethic Commission (File number 17-1986, “Commission d'éthique de l'utilisation des animaux de l'Université de Liège").

\section{- Laser microdissection of the choroid}

Mice subjected or not to CNV induction were sacrificed at D5 or D7 post-laser burn (n = 5-10 per experimental group, $4 \mathrm{CNV}$ lesions per eye). Eyes were enucleated, placed fresh in Tissue-Tek OCT medium (VWR, USA) and stored at $-80{ }^{\circ} \mathrm{C}$ until section preparation and laser microdissection. The day of laser microdissection, eyes were placed on dry ice until cryosection (Cryostat Cryotome FSE, Thermo Scientific). Ten $\mu \mathrm{m}$ sections were performed and place on PEN-Frame slides (Leica, Germany). Regions of interest (ROIs) were microdissected with Leica Laser Microdissection LMD 7000 and directly collected in lysis buffer for subsequent RNA extraction. Samples were collected from 3 different ROIs: control choroid (from control mouse not subjected to CNV), CNV lesion (neovascular tissue from laser-induced mouse) and their corresponding adjacent choroid (healthy tissue next to CNV lesion from laser-induced mouse). For each ROI, microdissected tissues from the two eyes of a single mouse were pooled before RNA extraction.

\section{- Intravitreal injections and optical coherence tomography (OCT)}

To assess inhibition/overexpression of miR-142-3p, intravitreal injections of inhibitor (single stranded LNA-antimiR) or mimic (chemically modified double-stranded) were performed in mice, just after laser induction (D0, inhibitor conditions), or just after laser induction and 5 days later (D0 + D5, mimic conditions) $(n=11-15$ per experimental group for flat-mounted choroids; $n=5-10$ per experimental group for flat-mounted retinas). During general anesthesia, mouse eyes were locally anesthetized with Unicaïn $0.4 \%$ (Théa Pharma) before the injection, in the vitreous chamber, of a solution $(2 \mu \mathrm{L})$ of inhibitor $(1.83 \mathrm{mM})$ or mimic $(10 \mu \mathrm{M})$. Negative control inhibitor and miR-142-3p inhibitor were purchased from Qiagen (miRCURY LNA miRNA Inhibitors, The Netherlands). Mimics (negative control and miR-142-3p mimic) were from Ambion (mirVana miRNA Mimics, USA). After intravitreal injection, eyes were covered with Trafloxal (Bausch + Lomb, Austria) to avoid 
any infection and inflammation. Both eyes of a single mouse received the same treatment. Phenotypic alterations consequent to miR-142-3p modulation were assessed through optical coherence tomography (OCT) measurements by using the MicronIV instrument, through vascular and microglia stainings on flat-mounted choroids, and through microglia staining on flat-mounted retinas. OCT measurements are presented as $(b-c) / c$ ratios, where $b$ is the CNV lesion thickness and $\mathrm{c}$ the adjacent choroid thickness [7]. For miR-142-3p mimic conditions, FITC-dextran (FD2000S, Sigma, USA) tail vein injections were performed 3 minutes before sacrifice.

\section{- RNA extraction, Reverse transcription and qRT-PCR}

Laser-captured choroids or cells were homogenized in lysis buffer by vortexing. For some qRT-PCR, whole retinas and choroids were lysed with ceramic beads (MagNA Lyser Green beads, Roche, Switzerland) in lysis buffer with tissue homogenizer (Precellys Evolution, Bertin, France). RNA was then extracted with miRNeasy Micro kit (lasercaptured choroids) (Qiagen) or with mirVana kit (all other experiments) (Ambion), according to the manufacturer's protocols. Complementary DNA was synthesized with miScript RT Kit II (Qiagen) using the same RNA input according to the manufacturer's instructions. mRNA levels of different targets and a set of microRNA levels were quantified by qRT-PCR using FastStart SYBR Green Master (Roche) and a LightCycler 96 instrument (Roche). MiRNA levels were assessed with miRCURY LNA miRNA PCR Assay (Qiagen). All data were normalized to GAPDH and snU6 expression as housekeeping genes. Primer sequences used to detect mouse and human target mRNAs are presented in Table $\mathbf{1}$.

5' - sequence - 3'

\begin{tabular}{|c|c|}
\hline \multirow{2}{*}{ mmu-GAPDH } & f: ggtggacctcatggcctaca \\
\hline \multirow{2}{*}{ mmu-uPA } & r: ctctcttgctcagtgtccttgct \\
\hline \multirow{2}{*}{ mmu-hsa-snU6 } & f: taaaatgctgtgtgctgcgg \\
\cline { 2 - 2 } & r: gcggccttcgatgttacaga \\
\hline \multirow{2}{*}{ hsa-GAPDH } & f: cgcttcggcagcacatatac \\
\hline \multirow{2}{*}{ hsa-CD68 } & r: ttcacgaatttgcgtgtcat \\
\hline \multirow{2}{*}{ hsa-VEGFa } & f: acccactcctccacctttgac \\
\hline & r: acctgttgctgtagccaatt \\
\hline & f: cagggaatgactgtcctcaca \\
\hline
\end{tabular}

\section{Table 1}

Forward (f) and reverse (r) primer sequences for mouse (mmu) and human (hsa) target mRNAs. 


\section{- Western blotting}

Cells were lysed with $1 \mathrm{X}$ lysis buffer (9803, Cell Signaling, USA) containing protease and phosphatase inhibitors (Complete and Phos-STOP, Roche). Proteins (15 $\mu \mathrm{g}$ ) were separated on acrylamide gels and transferred onto PVDF membranes. BCLAF1 proteins were detected by overnight incubation, at $4{ }^{\circ} \mathrm{C}$, with recombinant anti-BTF antibody $(1 / 10000$ dilution) (Ab181240, Abcam, UK) followed by $1 \mathrm{~h}$ incubation, at room temperature, with horseradish peroxidase-coupled secondary antibody (7074, Cell signaling) and enhanced chemiluminescent substrate (NEL1040001EA, PerkinElmer, USA) using a LAS4000 imager (Fujifilm). GAPDH (1/10 000, MAB 374, Millipore, USA) was used as a loading control.

\section{- Immunohistochemistry}

For mouse microglia immunostaining, eyes were enucleated and fixed in paraformaldehyde $4 \%$ for 1 hour. Subsequently, retinas and choroids were isolated, permeabilized, blocked and incubated with anti-Ibal antibody (1/1000 dilution) (Abcam Ab178846) overnight, at room temperature. The next day, samples were washed, incubated with secondary antibody (1/200 dilution) (goat anti-rabbit AlexaFluor 595, Invitrogen A11012, USA) for 2 hours and washed again. Retinas and choroids were flat-mounted with Fluoromount-G (SouthernBiotech, The Netherlands) on glass-slides for microscopy imaging.

For mouse vascular immunostaining, eyes were enucleated and fixed in ethanol $70 \%$ for 1 hour. Subsequently, choroids were isolated, permeabilized, blocked and incubated with anti-CD31 antibody (1/150 dilution) (Pharmingen 553370) overnight, at room temperature. The next day, samples were washed, incubated with secondary antibody (1/200 dilution) (goat anti-rat AlexaFluor 488, Invitrogen A11006) for 2 hours and washed again. Choroids were flat-mounted with Fluoromount-G (SouthernBiotech) on glass-slides for microscopy imaging.

- Epifluorescence/confocal microscopy and image quantifications

Flat-mounted Iba1/CD31 stained choroids were observed under an Olympus Vanox epifluorescence microscope (inhibitors conditions). Flat-mounted Iba1/FITC stained choroids were observed under a Leica Sp5 confocal microscope (mimics conditions) and Zstacks (z-path $=2.5 \mu \mathrm{m}$ ) were taken. Each CNV lesion was separately imaged and maximum intensity projections were performed for Iba1/FITC images. For each CNV lesion, the area occupied by the staining was calculated with Fiji software [21]. The mean area for a single eye was than calculated for each staining. The percentage of stained area within an eye, normalized to control condition, was used for subsequent statistical analysis to determine vascular and microglia densities.

For cell solidity analysis, Z-stacks of flat-mounted Ibal stained retinas were taken with a high-resolution confocal microscope (Zeiss LSM 880 with Airyscan), at the 20X magnification. Every CNV lesion was separately imaged, each image representing a $780 \mu \mathrm{m}$ long square, englobing one $\mathrm{CNV}$ area and the surrounding tissue. After acquisition, images were first processed and then quantified. The processing procedure started by identifying 
image background with morphological opening operations and subtracting it from the raw images. They were then thresholded using Otsu's method and subjected to a median filter to reduce noise. First quantification steps consisted in detecting every objects of the image and discarding the ones which were too small/big to be an isolated cell or a small cell cluster. The bright center of the cells was then used to segment and isolate each cell. Remaining noncellular objects were discarded using shape and size filters. Finally, tridimensional solidity was measured for every cell. The mean cell solidity of all the CNV lesions area of a single eye was calculated and then used for subsequent data analysis. The solidity of an object is defined as the ratio of the volume of this object over its convex volume. The convex volume corresponds to the volume of the convex hull of the region, i.e. the smallest region that satisfy two conditions: (1) it is convex (2) it contains the original region.

- Cell culture

Human microglia HMC3 cells were purchased from ATCC (CRL-3304, USA) and cultured according to the manufacturer's instructions. Cells were seeded in 6-well plate $\left(2.10^{5}\right.$ cells per well) the day before transfection or stimulation. HMC3 were transfected with $5 \mathrm{nM}$ of miR-142-3p inhibitor/negative control inhibitor or with $5 \mathrm{nM}$ miR-142-3p mimic/negative control mimic. Transfection was performed using INTERFERin reagent (Polyplus transfection) following manufacturer's protocol. Cells were harvested 24-hour post-transfection to assess miR-142-3p, BCLAF1 transcript and VEGF-A levels, or 48-hour and 72-hour to assess CD68 and BCLAF1 protein levels, respectively. Alternatively, HMC3 underwent pro-inflammatory stimulation by a 24 hours exposure to $10 \mathrm{ng} / \mathrm{mL}$ IFN $\gamma$ (Recombinant Human IFN $\gamma$, Peprotech, USA) or to $0,1 \mu \mathrm{g} / \mathrm{mL}$ LPS (Lipopolysaccharides from Escherichia coli 0111:B4, Sigma, USA).

- Data analysis

All data and measurements underwent a statistical analysis using Prims7 software. All graphs are presented as mean +/- SEM. Mann-Whitney test or Kruskal-Wallis followed by post-hoc multiple comparisons tests were used to determine statistical significance. Statistical significance was defined as $\mathrm{p} \leq 0.05$. 


\section{Results}

\section{MiR-142-3p is overexpressed in a laser-induced CNV mouse model}

Laser microdissection was used to isolate three types of samples from mice subjected or not to laser-induced CNV: i) control choroid from control mice (not subjected to CNV induction), ii) $\mathrm{CNV}$ lesions, and iii) adjacent choroid (healthy choroid adjacent to $\mathrm{CNV}$ lesion) (Fig. 1A). In a pilot experiment, the expression profile of 15 angiogenesis and/or inflammation-related microRNAs were determined 7 days post-induction by qRT-PCR in the 3 regions of interest (Fig. 1B). Although probably not extensive enough, we focused our attention only on these 15 microRNAs i) because of limited RNA availability after laser microdissection and ii) because these microRNAs are reported angiogenesis and/or inflammation mediators. Among those microRNAs tested, only 4 were dysregulated: miR21-5p, miR-34a-5p and miR-142-3p increased whereas miR-574-3p decreased upon laserinduction. To further confirm the overexpression of miR-142-3p in laser-induced CNV mouse, laser microdissection was repeated 7 days as well as 5 days post laser-induction. Interestingly, miR-142-3p increased in the CNV lesions, both at days 5 and 7 post laser induction (Fig. 1C), and correlated with the overexpression of uPA (urokinase plasminogen activator) used here as a CNV-disease marker [22] (Fig. 1D and Supp. 1). Taken together, these data suggest that miR-142-3p could act during CNV progression in the laser-induced CNV mouse model. 

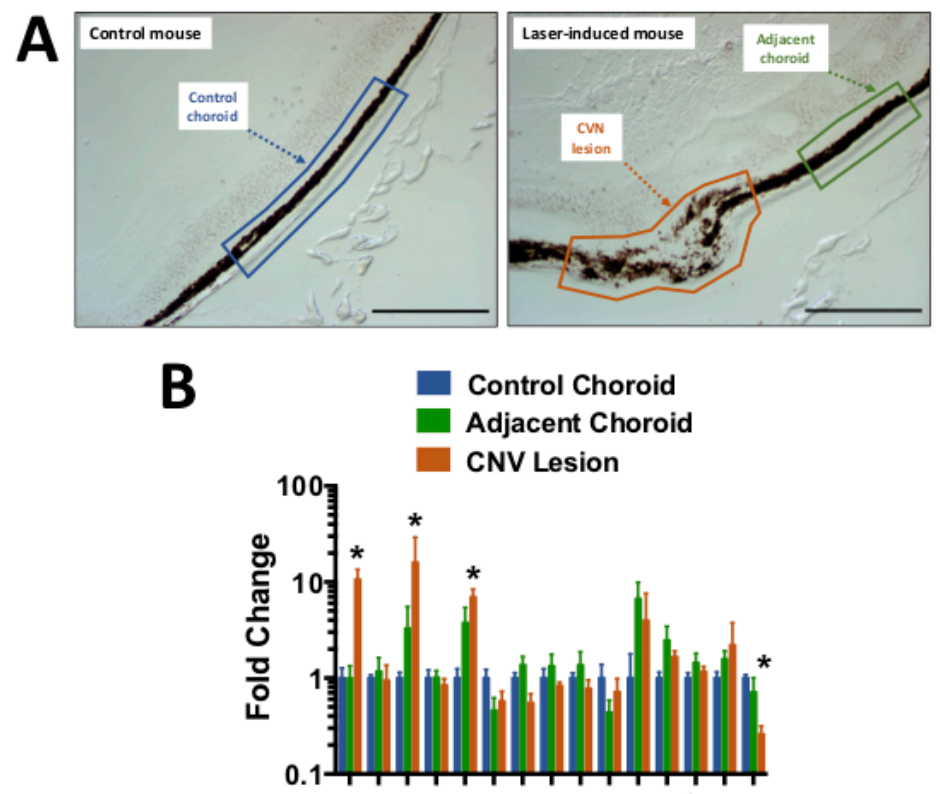

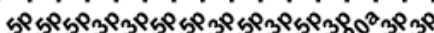

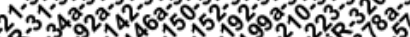

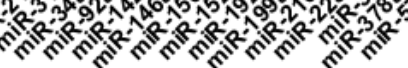

$\mathrm{C}$

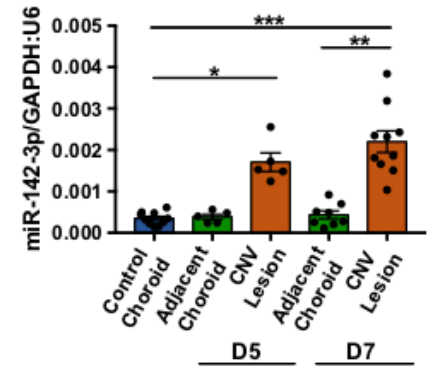

D

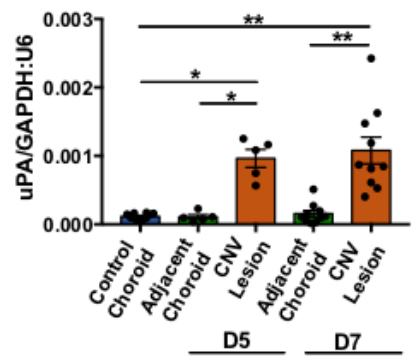

Figure 1. MiR-142-3p is overexpressed in a laser-induced CNV mouse model.

A Cryosections of control mouse (untreated) and laser-induced mouse delineating the different regions of interest (ROI). These ROIs, namely control choroid (in blue), adjacent choroid (in green) and CNV lesion (in orange), were isolated by laser microdissection. Scale bar $=250 \mu \mathrm{m}$. B MicroRNA expression in CNV lesion and adjacent choroid compared to control choroid, 7 days after laser induction. Results of CNV lesion and adjacent choroid are expressed as fold change to control choroid. Results are presented as mean +- SEM. Oneway ANOVA followed by multiple comparisons test $(\mathrm{n}=5$ per experimental group). C\&D Overexpression of miR-142-3p (C), and uPA (D), a CNV-disease marker, at day 5 (D5) and day 7 (D7). MiR-142-3p and uPA are specifically overexpressed in CNV lesion 5 and 7 days post laser induction. qRT-PCR results are presented as mean +- SEM. One-way ANOVA + multiple comparisons test $(\mathrm{n}=10$ for control choroid, $\mathrm{n}=5$ for adjacent choroid and CNV lesion D5, $\mathrm{n}=10$ for adjacent choroid and CNV lesion D7) $(*=\mathrm{p} \leq 0.05 ; * *=\mathrm{p} \leq 0.01 ; * * *$ $=\mathrm{p} \leq 0.001)$ 


\section{Modulation of miR-142-3p expression alters neovascularization and inflammation}

in $\mathrm{CNV}$ mouse model

Intravitreal injections of miR-142-3p inhibitor or mimic allowed us to evaluate the functional role of miR-142-3p during CNV progression in the laser-induced CNV mouse model. CNV lesion thickness, as assessed by OCT measurements, was lower in mice injected with miR-142-3p inhibitor when compared to control inhibitor (Fig. 2A) $(p=0.0149)$. This inhibitor reduced by $46 \%$ the blood vessel density in CNV mouse (Fig. 2B, top panels) ( $\mathrm{p}=$ 0.0157). Concomitantly, microglia area, a major component of the innate immunity in the eye, was decreased by $30 \%$ upon miR-142-3p inhibition (Fig. 2B, bottom panels) ( $\mathrm{p}=$ 0.0292). Therefore, miR-142-3p inhibition alleviates CNV progression by decreasing neovascularization and inflammation. Inversely, the overexpression of miR-142-3p through mimic injection slightly enhanced CNV lesion thickness (Fig. 3A) $(\mathrm{p}=0.0407)$. It also increased the angiogenic response by $136 \%$ (Fig. 3B, top panels) $(p=0.0233)$ and microglia cell recruitment by $126 \%$ (Fig. 3B, bottom panels) $(\mathrm{p}=0.0441)$. For both inhibitor and mimic conditions, miR-142-3p retinal levels were measured to assess its inhibition/overexpression (Supp. 2 \& Supp. 3). Altogether, the modulation of miR-142-3p expression in $\mathrm{CNV}$ mouse model revealed a functional role of this microRNA during CNV progression. Intriguingly, both the vascular and microglia components of CNV lesions were affected by miR-142-3p modulation. 

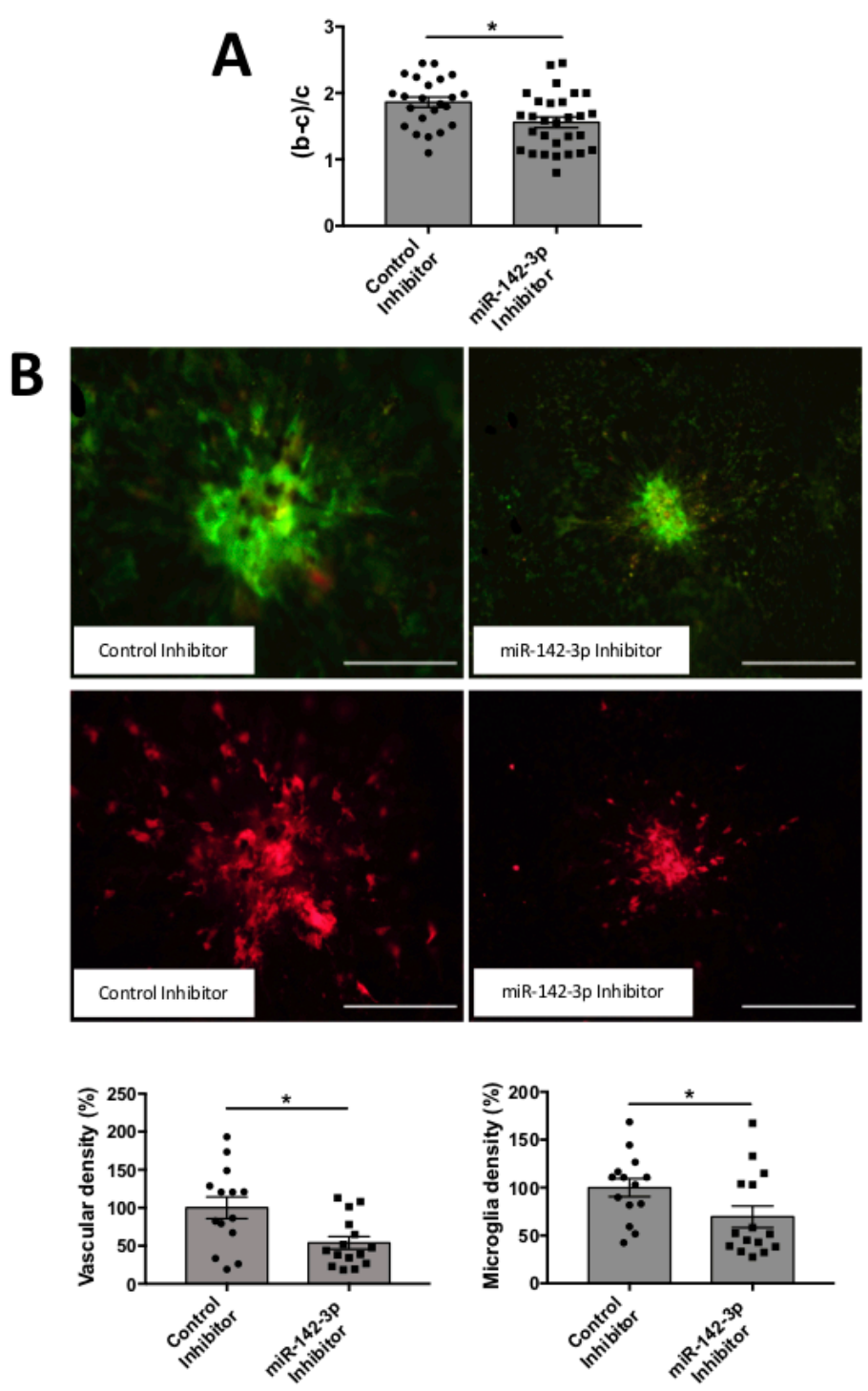

Figure 2. Inhibition of miR-142-3p in a laser-induced CNV mouse model decreases both vascular and inflammatory phenotypes.

A OCT measurement presented as (b-c)/c ratios where $\mathrm{b}$ is the $\mathrm{CNV}$ lesion thickness and $\mathrm{c}$ is the adjacent choroid thickness. $\mathrm{b}$ and $\mathrm{c}$ were measured just prior to sacrifice $(\mathrm{n}=23-29$ per experimental group). B Flatmounted choroids showing vascular (in green) and microglia (in red) density and corresponding quantification. Scale bar $=250 \mu \mathrm{m}(\mathrm{n}=14-15$ per experimental group $)$. All results are presented as mean + SEM. Mann Whitney test $(*=\mathrm{p} \leq 0.05)$ 

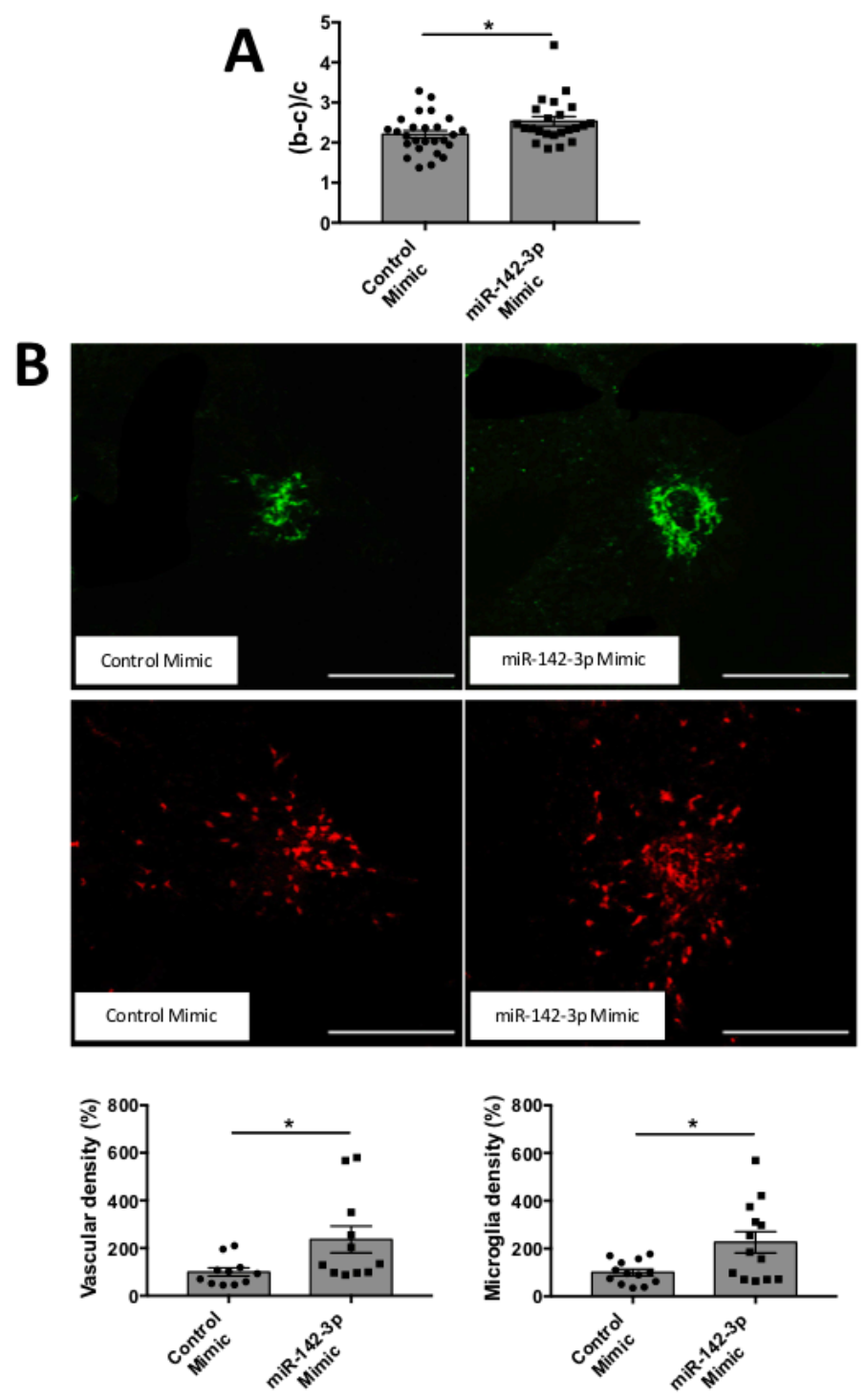

Figure 3. Overexpression of miR-142-3p in a laser-induced CNV mouse model increases both vascular and inflammatory phenotypes.

A OCT measurement presented as (b-c)/c ratios where $\mathrm{b}$ is the CNV lesion thickness and $\mathrm{c}$ is the adjacent choroid thickness. $\mathrm{b}$ and $\mathrm{c}$ were measured just prior to sacrifice $(\mathrm{n}=24-26$ per experimental group). B Flatmounted choroids showing vascular (in green) and microglia (in red) density and corresponding quantification. Scale bar $=250 \mu \mathrm{m}(\mathrm{n}=11-13$ per experimental group $)$. All results are presented as mean + SEM. Mann Whitney test $(*=p \leq 0.05)$ 


\section{Retina microglia cell activation is closely linked to miR-142-3p expression}

Microglia cells are resident immune cells of the central nervous system, including the retina. In laser-induced mouse model of $\mathrm{CNV}$, microglia are recruited from the retina and invade the underlying $\mathrm{CNV}$ lesion [23, 24]. Under activation in inflammatory conditions, resting microglia cells lose their highly ramified morphology and gain an amoeboid shape. To assess the effect of miR-142-3p on retina microglia cell shape in vivo, an innovative computerized quantification method has been set up. Cell solidity corresponds to the ratio between the cell volume and its convex volume, and reflects microglia activation (Fig 4A). While activated amoeboid microglia cells are characterized by a solidity closer to 1 (which characterizes a round cell), resting ramified microglia cells are characterized by a solidity closer to 0 (Fig. 4A). Each portion of the whole retina corresponding to a CNV lesion and its surrounding tissue was imaged at the $20 \mathrm{X}$ magnification to insure an optimal resolution for cell solidity analysis (Fig. 4B). Raw images were processed and binarized for computerized quantification, which allowed a clear discrimination of every single microglia cell within the entire image (Fig. 4C and Supp. 4). Interestingly, this computerized image analysis revealed that miR-142-3p inhibitor and miR-142-3p mimic impacted drastically the morphology of retina microglia cell (Fig. 4D). While miR-142-3p inhibitor decreased microglia solidity and thus microglia activation, miR-142-3p mimic had the opposite effect. Altogether, these data suggest that miR-142-3p contributes in vivo to microglia cell activation. 

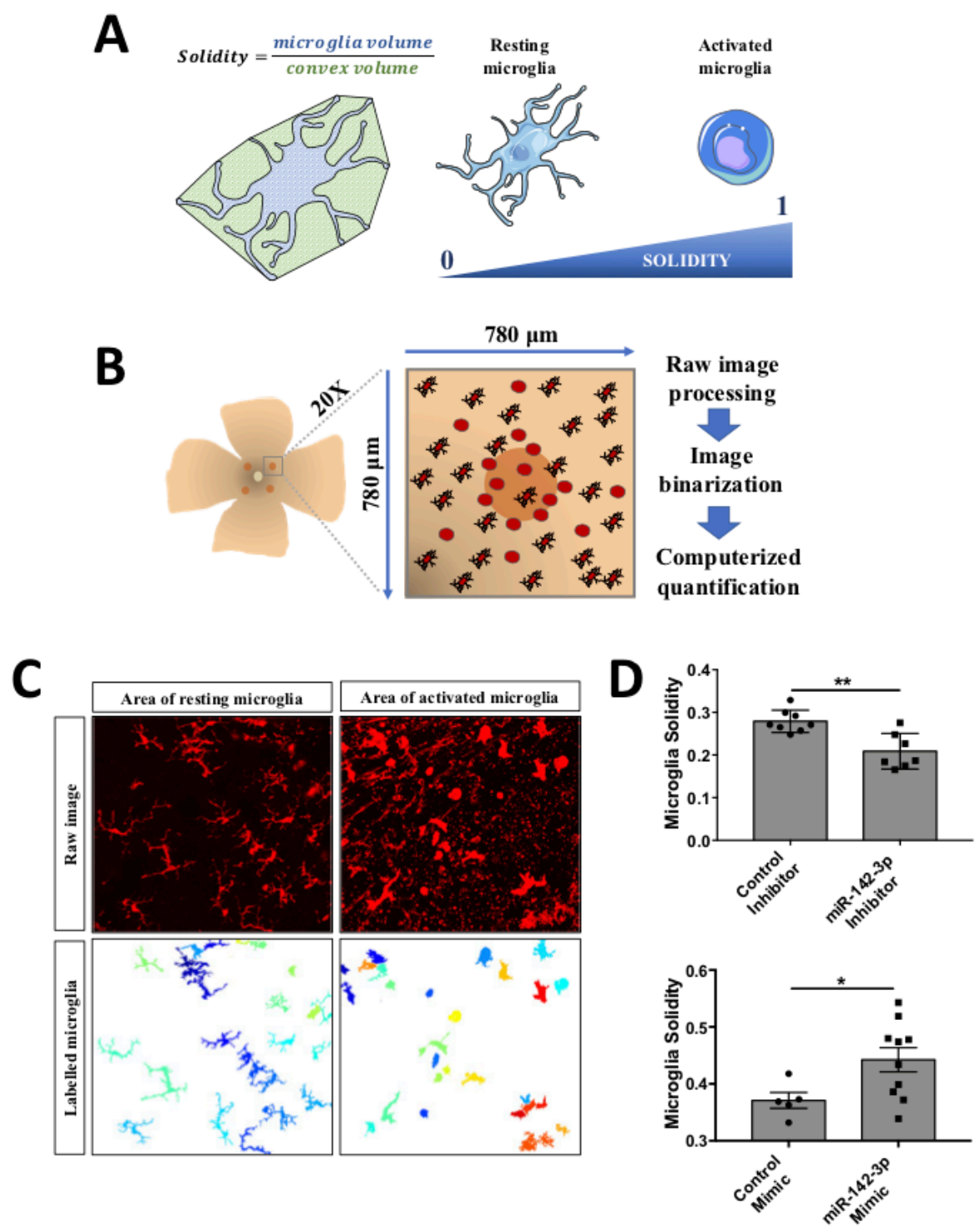

Figure 4. MiR-142-3p influences microglia cell activation state in vivo.

A Characterization of microglia morphology via cell solidity. The solidity of an object is defined as the ratio between its volume and its convex volume. Resting microglia are highly ramified while activated microglia present an amoeboid shape, with no or small ramifications. Activated microglia are characterized by a higher solidity. B CNV lesion area of flat-mounted retinas and surrounding healthy tissue were imaged at the $20 \mathrm{X}$ magnification and then processed and quantified. C Representative raw images and corresponding labelled images of resting and activated microglia area. D Microglia activity measured around the CNV lesion in mice injected with either miR-142-3p inhibitor or mimic and relative controls $(n=5-10$ per experimental group). All results are presented as mean + - SEM. Mann Whitney test $(*=p \leq 0.05 ; * *=p \leq 0.01)$ 


\section{MiR-142-3p regulates the activation of human microglia cells under pro- inflammatory conditions}

Transfection of human microglia HMC3 cells with miR-142-3p mimic drastically increased miR-142-3p expression (Fig. 5A, right panel). It also decreased the production of its previously described target, BCLAF1, both at mRNA (Fig. 5B, right panel) and protein (Fig. 5C) levels [25]. BCLAF1 was used here to validate the efficacy of miR-142-3p mimic. Alongside with its effect on BCLAF1 expression, miR-142-3p overexpression also influenced HMC3 activation state as assessed by increased CD68 level (Fig. 5D, right panel), a marker of microglia activation [26, 27]. Interestingly, VEGF-A transcript level (Fig. 5E, right panel) increased upon miR-142-3p mimic, which could contribute to the effect observed in vivo on the angiogenic response. When using the inhibitor, only a slight decrease of miR142-3p expression was observed (Fig. 5A, left panel) without any effect on BCLAF1, CD68 and VEGF-A levels (Fig. 5 B-E, left panels). Such a low effect of the inhibitor may be related to the low basal level of miR-142-3p in HMC3 cell line.

Our data in vitro confirm the in vivo findings of the biological implication of miR-142$3 p$ in microglia activation. This is further supported by the concomitant increase of miR-142$3 p$ and CD68 expressions in HMC3 cells stimulated by IFN $\gamma$ or LPS (Fig. 5F). Taken together, these data revealed a strong link between the activation state of microglia cell and miR-142-3p expression. 


\section{A}
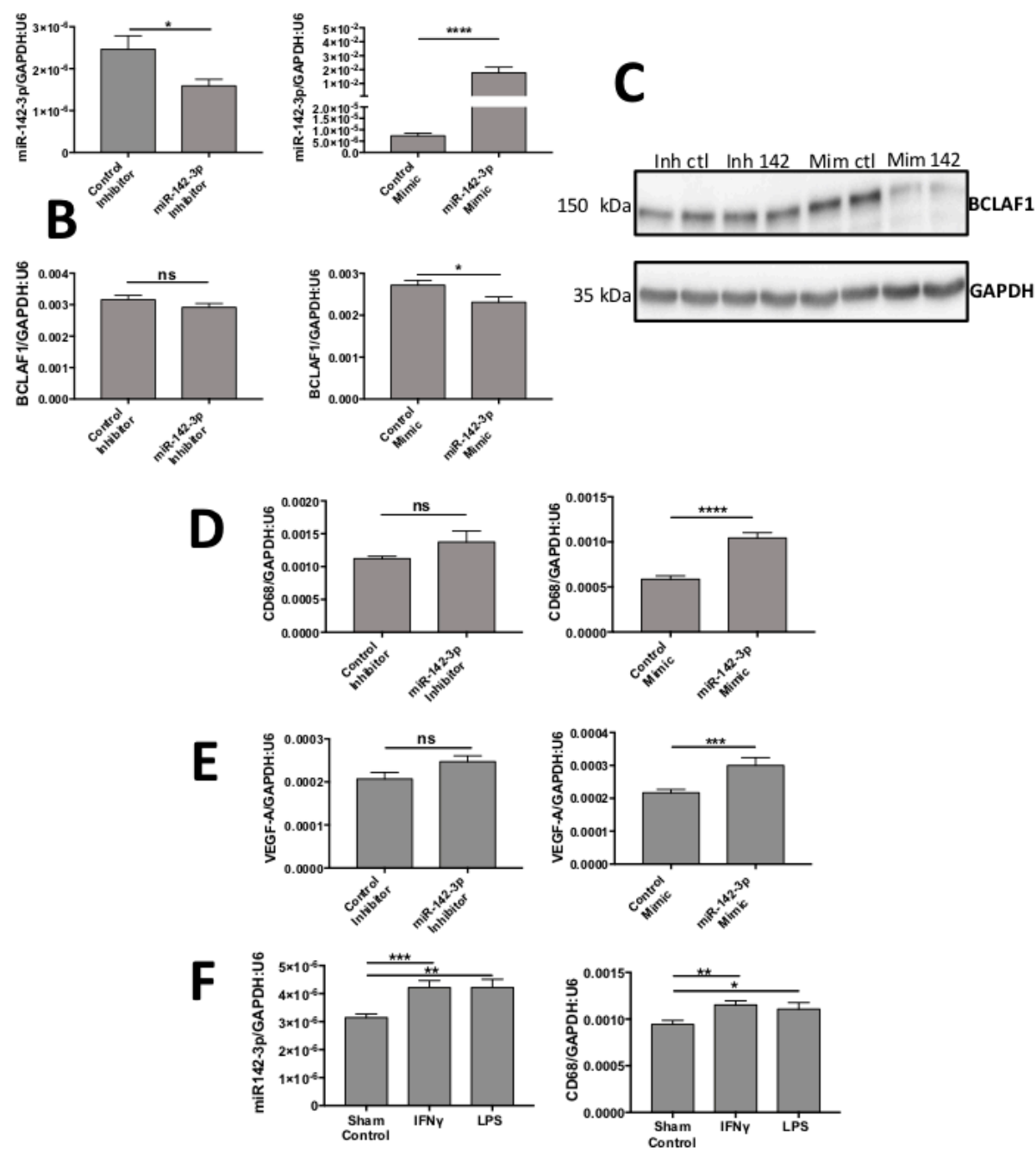

Figure 5. MiR-142-3p regulates the activation of human microglia cells under proinflammatory conditions.

A-C Transfections of miR-142-3p inhibitor and mimic lead to decreased and increased miR-142-3p level, respectively (A). Transfection of miR-142-3p mimic decreased BCLAF1 levels, a previously identified target of miR-142-3p, both at the transcript level (B) and protein level (C). D-E Effects on CD68 level (D) or VEGFA (E) in HMC3 transfected with miR-142-3p inhibitor or mimic and relative controls. Only miR-142-3p mimic was able to induce CD68 et VEGF-A production. F HMC3 stimulated with either IFN $\gamma$ or LPS for 24 hours overexpress miR-142-3p as long with CD68.All qRT-PCR results are presented as mean +- SEM. Mann Whitney test. $(*=\mathrm{p} \leq 0.05 ; * *=\mathrm{p} \leq 0.01 ; * * *=\mathrm{p} \leq 0.001 ; * * * *=\mathrm{p} \leq 0.0001 ; \mathrm{ns}=$ not significant $)$ 


\section{Discussion}

The present study unveils a functional role of miR-142-3p in stimulating CNV formation in a mouse model of wet AMD. This novel concept is supported by i) the overexpression of miR-142-3p in CNV lesions, and by ii) the impact of this miRNA modulation (inhibition/overexpression) on both vascular and inflammatory phenotypes. While inhibition of miR-142-3p caused a subsequent decrease of angiogenesis, its overexpression had the opposite effect. Beside its effect on angiogenesis, administration of miR-142-3p activates microglia cells -part of the inflammatory process- both in vivo and in vitro. Our data provide evidence for a pivotal role played by miR-142-3p during CNV progression and particularly on microglia activation.

The originality of our approach relies on the laser microdissection, which enables us to isolate CNV tissue devoid of any surrounding healthy tissue that could have biased the microRNA profile [28]. Indeed, previous studies in CNV mouse primarily investigated microRNAs circulating in the blood $[17,29]$ or present in the entire RPE-choroid-sclera complexes [30]. It is noteworthy that CNV lesion sites only englobe $2.5 \%$ of total choroid tissue, making entire choroid complexes highly heterogenous mixes of healthy and nonhealthy tissue. Among the microRNAs tested, 4 were dysregulated: miR-21-5p, miR-34a-5p and miR-142-3p increased, whereas miR-574-3p decreased upon laser-induction. In line with our data, miR-21-5p was previously reported as strongly up-regulated in RPE-choroid-sclera complex [30]. MiR-21-5p is a well-known pro-angiogenic microRNA in different pathologies [31, 32] and is highly abundant in human retinal endothelial cells [33]. Concomitantly to the overexpression of miR-34a-5p in our samples, this microRNA was found to be upregulated in serum of AMD patients [34] and in hydrogen peroxide-induced prematurely senescent ARPE-19 cells [35], possibly leading thus to AMD progression.

Here, we identified miR-142-3p as a specific CNV lesion microRNA, being upregulated both 5- and 7-days post laser-induction and displaying a correlation with uPA, a wellestablished CNV disease marker [22]. MiR-142 gene is a broadly conserved miRNA gene among species that gives rise to two distinct mature forms, miR-142-3p and miR-142-5p. MiR-142 is involved in stem cell fate, cardiomyocyte hypertrophy, cancer, immune tolerance and hematopoiesis, as previously reviewed [36]. Both vascular [25] and inflammatory [37] functions have been attributed to this microRNA. Interestingly, miR-142-3p expression is upregulated in a mouse model of retinal degeneration [38], but its biological role in ocular disease, including wet AMD, remained unknown. The increase of miR-142-3p in CNV goes in parallel with macrophage recruitment, as well as the increase in vascular density [7]. Thus, miR-142-3p expression was associated with two interconnected hallmarks of the disease, namely inflammation and angiogenesis. Previous studies revealed that the cellular source of miR-142-3p may be more immune-related rather than vascular-related [39-41]. Accordingly, miR-142-3p expression in human microglia cells increased upon IFN $\gamma$ or LPS stimulation, and correlated with CD68 level, a marker of activated microglia. To evaluate the functional role of miR-142-3p during CNV, miR-142-3p inhibitor or mimic were injected into the vitreous chamber of $\mathrm{CNV}$ mouse. Intravitreal injections are routinely used by clinicians to 
deliver anti-VEGF drugs to wet AMD patients [42]. On one hand, intravitreal injection of miR-142-3p inhibitor decreased the vascular density in flat-mounted choroids, while injection of miR-142-3p mimic conversely increased it. Those findings were further confirmed by alterations of CNV lesion thickness measured via OCT. Furthermore, intravitreal miR-142-3p inhibition alleviated microglia density, while miR-142-3p mimic increased it.

Microglia are the immune resident cells of the central nervous system, constantly screening their environment for pathogens and ready to migrate, proliferate and phagocyte if needed. In physiological conditions, retinal microglia cells exhibit a highly ramified morphology, shifting to a more amoeboid shape under activation [24]. In the laser-induced $\mathrm{CNV}$ context, microglia are known to migrate from retina and invade CNV lesion site, starting 4-days post laser-induction [23]. Various cellular morphology parameters exist to describe microglia shape [43, 44], but cell solidity has been described as the most appropriate one for assessing the amoeboid shape of activated microglia, and thus microglia activation [45]. An innovative computerized imaging and quantification technique revealed that miR142-3p inhibition/overexpression decreases/increases retinal microglia cell solidity in vivo, in line with our in vitro data of miR-142-3p mediated microglia activation. Our data suggest that miR-142-3p stimulates the activation of microglia in the retina and their migration to the $\mathrm{CNV}$ lesion site, located within the choroid. Accordingly, miR-142-3p is one of the top upregulated microRNA in primary microglia isolated from rat pup's brain under LPS stimulation [46]. Besides morphological analysis, future studies may evaluate mouse microglia activation state through transcriptomic and surface marker analyses. Interestingly, in human microglia culture, miR-142-3p mimic enhanced drastically miR-142-3p levels, as well as CD68 expression, a marker of activated microglia [26, 27]. An interesting finding is the concomitant up-regulation of VEGF-A, a key molecular mediator of CNV progression [47]. This demonstrates that miR-142-3p is able to modulate both inflammatory and vascular responses, two intimately linked processes associated to $\mathrm{CNV}$ progression [48]. Interestingly, CD68 was also upregulated in HMC3 stimulated by IFN $\gamma$ or LPS, leading to a pro-inflammatory state $[49,50]$. This observation supports the concept that miR-142-3p is a mediator of inflammation triggering microglia cell activation and is in line with our in vivo data. How miR-142-3p modulates CD68 and VEGF-A production remains to be elucidated. However, our data support the concept that miR-142-3p could be a new intermediate in the vascular-inflammatory axis during $\mathrm{CNV}$ progression, through microglia activation.

In conclusion, miR-142-3p may exacerbate $\mathrm{CNV}$ by enhancing microglia migration and activation, and therefore is worth considering as a potential therapeutic target in AMD. 


\section{Acknowledgments}

The authors kindly acknowledge Nathalie Lefin and Erika Konradowski for technical support and the GIGA Flow Cytometry and Cell Imaging Platform for their help for image acquisition and laser microdissection. This study was supported by a joined $\mathrm{PhD}$ funding program in the framework of the cooperation between the University of Liège and Maastricht University named "Towards a joint imaging valley". Financial supports from Fondation Léon Fredericq, FEDER (BIOMED HUB - DMLA-AB), FNRS-PDR (grant number: T.1080.15) and "Fonds Spéciaux de l'Université de Liège" are gratefully acknowledged. This study has also been possible thanks to the support of the ERA-Net-CVD project MacroERA, 01KL1706. The authors acknowledge the support from the Netherlands Cardiovascular Research Initiative, an initiative with support of the Dutch Heart Foundation, CVON2016Early HFPEF, 2015-10, CVON She-PREDICTS, grant 2017-21, CVON Arena-PRIME, 2017-18.

\section{Conflicts of interest}

The authors declare no conflicts of interest.

\section{Authors' Contributions}

Q.R. conceptualized the work, performed investigations, interpreted results and wrote the manuscript. J.L. conceptualized the work, performed investigations and reviewed and/or edited the manuscript. T.L., C.Y., L.B. and V.L. performed investigations and reviewed and/or edited the manuscript. V.C. and I.S. reviewed and/or edited the manuscript. A.N. and S.H. conceptualized the work, supervised the study and reviewed and/or edited the manuscript. 


\section{References}

1. $\quad$ WHO, World report on vision. 2019.

2. Flaxman, S.R., et al., Global causes of blindness and distance vision impairment 1990-2020: a systematic review and meta-analysis. The Lancet Global Health, 2017. 5(12): p. e1221-e1234.

3. Wong, W.L., et al., Global prevalence of age-related macular degeneration and disease burden projection for 2020 and 2040: a systematic review and metaanalysis. The Lancet Global Health, 2014. 2(2): p. e106-e116.

4. Adelman, R.A., E.J. Sigler, and R.K. Seth, Age-related Macular DegenerationReview and Current Concepts. US Ophthalmic Review, 2011. 04(01).

5. Storey, P., L. Hark, and J.A. Haller, Age-Related Macular Degeneration, in Handbook of Nutrition, Diet and the Eye. 2014. p. 11-20.

6. Fletcher, E.L., Contribution of microglia and monocytes to the development and progression of age related macular degeneration. Ophthalmic Physiol Opt, 2020. 40(2): p. 128-139.

7. Lambert, V., et al., Laser-induced choroidal neovascularization model to study age-related macular degeneration in mice. Nat Protoc, 2013. 8(11): p. 2197-211.

8. $\quad$ Pennesi, M.E., M. Neuringer, and R.J. Courtney, Animal models of age related macular degeneration. Mol Aspects Med, 2012. 33(4): p. 487-509.

9. Park, Y.G., et al., New Approach of Anti-VEGF Agents for Age-Related Macular Degeneration. J Ophthalmol, 2012. 2012: p. 637316.

10. Zuber-Laskawiec, K., et al., Non-responsiveness and tachyphylaxis to antivascular endothelial growth factor treatment in naive patients with exudative agerelated macular degeneration. J Physiol Pharmacol, 2019. 70(5).

11. O'Brien, J., et al., Overview of MicroRNA Biogenesis, Mechanisms of Actions, and Circulation. Front Endocrinol (Lausanne), 2018. 9: p. 402.

12. Gebert, L.F.R. and I.J. MacRae, Regulation of microRNA function in animals. Nat Rev Mol Cell Biol, 2019. 20(1): p. 21-37.

13. Bartel, D.P., Metazoan MicroRNAs. Cell, 2018. 173(1): p. 20-51.

14. $\mathrm{Xu}, \mathrm{S}$. and L.D. Hazlett, MicroRNAs in Ocular Infection. Microorganisms, 2019. 7(9).

15. Raghunath, A. and E. Perumal, Micro-RNAs and their roles in eye disorders. Ophthalmic Res, 2015. 53(4): p. 169-86.

16. Ménard, C., et al., MicroRNA signatures in vitreous humour and plasma of patients with exudative AMD. Oncotarget, 2016. 7(15): p. 19171-84.

17. Kiel, C., et al., A Circulating MicroRNA Profile in a Laser-Induced Mouse Model of Choroidal Neovascularization. Int J Mol Sci, 2020. 21(8).

18. Liu, C.H., et al., MicroRNAs in Vascular Eye Diseases. Int J Mol Sci, 2020. 21(2).

19. Marques-Rocha, J.L., et al., Noncoding RNAs, cytokines, and inflammationrelated diseases. FASEB J, 2015. 29(9): p. 3595-611.

20. Tiwari, A., B. Mukherjee, and M. Dixit, MicroRNA Key to Angiogenesis Regulation: MiRNA Biology and Therapy. Curr Cancer Drug Targets, 2018. 18(3): p. 266-277.

21. Schindelin, J., et al., Fiji: an open-source platform for biological-image analysis. Nat Methods, 2012. 9(7): p. 676-82.

22. Rakic, J.M., et al., Mice without $u P A, t P A$, or plasminogen genes are resistant to experimental choroidal neovascularization. Invest Ophthalmol Vis Sci, 2003. 44(4): p. 1732-9. 
23. Crespo-Garcia, S., et al., In vivo analysis of the time and spatial activation pattern of microglia in the retina following laser-induced choroidal neovascularization. Exp Eye Res, 2015. 139: p. 13-21.

24. Silverman, S.M. and W.T. Wong, Microglia in the Retina: Roles in Development, Maturity, and Disease. Annu Rev Vis Sci, 2018. 4: p. 45-77.

25. Bao, H., et al., Platelet-derived microparticles promote endothelial cell proliferation in hypertension via miR-142-3p. FASEB J, 2018. 32(7): p. 39123923.

26. Costa, T., et al., Combined 1-Deoxynojirimycin and Ibuprofen Treatment Decreases Microglial Activation, Phagocytosis and Dopaminergic Degeneration in MPTP-Treated Mice. J Neuroimmune Pharmacol, 2020.

27. Stankov, A., et al., Visualisation of Microglia with the use of Immunohistochemical Double Staining Method for CD-68 and Iba-1 of Cerebral Tissue Samples in Cases of Brain Contusions. Pril (Makedon Akad Nauk Umet Odd Med Nauki), 2015. 36(2): p. 141-5.

28. Hernandez, S. and J. Lloreta, Manual versus laser micro-dissection in molecular biology. Ultrastruct Pathol, 2006. 30(3): p. 221-8.

29. Grassmann, F., et al., A circulating microrna profile is associated with late-stage neovascular age-related macular degeneration. PLoS One, 2014. 9(9): p. e107461.

30. Zhang, L., et al., Differential Expressions of microRNAs and Transfer RNAderived Small RNAs: Potential Targets of Choroidal Neovascularization. Curr Eye Res, 2019. 44(11): p. 1226-1235.

31. Du, X., et al., miR-21 induces endothelial progenitor cells proliferation and angiogenesis via targeting FASLG and is a potential prognostic marker in deep venous thrombosis. J Transl Med, 2019. 17(1): p. 270.

32. Fan, B., et al., MicroRNA-21 contributes to renal cell carcinoma cell invasiveness and angiogenesis via the PDCD4/c-Jun (AP-1) signalling pathway. Int J Oncol, 2020. 56(1): p. 178-192.

33. Walz, J.M., et al., Impact of angiogenic activation and inhibition on miRNA profiles of human retinal endothelial cells. Exp Eye Res, 2019. 181: p. 98-104.

34. Romano, G.L., et al., Retinal and Circulating miRNAs in Age-Related Macular Degeneration: An In vivo Animal and Human Study. Front Pharmacol, 2017. 8: p. 168.

35. Tong, N., et al., Involvement of microRNA-34a in Age-Related Susceptibility to Oxidative Stress in ARPE-19 Cells by Targeting the Silent Mating Type Information Regulation 2 Homolog 1/p66shc Pathway: Implications for AgeRelated Macular Degeneration. Front Aging Neurosci, 2019. 11: p. 137.

36. Shrestha, A., et al., MicroRNA-142 is a multifaceted regulator in organogenesis, homeostasis, and disease. Dev Dyn, 2017. 246(4): p. 285-290.

37. Mandolesi, G., et al., miR-142-3p Is a Key Regulator of IL-1beta-Dependent Synaptopathy in Neuroinflammation. J Neurosci, 2017. 37(3): p. 546-561.

38. Huang, P., et al., MicroRNA Expression Patterns Involved in Amyloid BetaInduced Retinal Degeneration. Invest Ophthalmol Vis Sci, 2017. 58(3): p. 17261735.

39. Chaudhuri, A.D., et al., Up-regulation of microRNA-142 in simian immunodeficiency virus encephalitis leads to repression of sirtuin1. FASEB J, 2013. 27(9): p. 3720-9. 
40. Carofino, B.L., et al., Head and neck squamous cancer progression is marked by CLIC4 attenuation in tumor epithelium and reciprocal stromal upregulation of miR-142-3p, a novel post-transcriptional regulator of CLIC4. Oncotarget, 2019. 10(68): p. 7251-7275.

41. Guiot, J., et al., Macrophage-derived exosomes attenuate fibrosis in airway epithelial cells through delivery of antifibrotic miR-142-3p. Thorax, 2020. 75(10): p. 870-881.

42. $\mathrm{Ba}, \mathrm{J}$., et al., Intravitreal anti-VEGF injections for treating wet age-related macular degeneration: a systematic review and meta-analysis. Drug Des Devel Ther, 2015. 9: p. 5397-405.

43. Young, K. and H. Morrison, Quantifying Microglia Morphology from Photomicrographs of Immunohistochemistry Prepared Tissue Using ImageJ. J Vis Exp, 2018(136).

44. Damani, M.R., et al., Age-related alterations in the dynamic behavior of microglia. Aging Cell, 2011. 10(2): p. 263-76.

45. Zanier, E.R., et al., Shape descriptors of the "never resting" microglia in three different acute brain injury models in mice. Intensive Care Med Exp, 2015. 3(1): p. 39.

46. Gupta, N., et al., miR-142-3p Regulates BDNF Expression in Activated Rodent Microglia Through Its Target CAMK2A. Front Cell Neurosci, 2020. 14: p. 132.

47. Kwak, N., et al., VEGF is major stimulator in model of choroidal neovascularization. Invest Ophthalmol Vis Sci, 2000. 41(10): p. 3158-64.

48. Huang, H., et al., VEGF receptor blockade markedly reduces retinal microglia/macrophage infiltration into laser-induced CNV. PLoS One, 2013. 8(8): p. e71808.

49. Martinez Leo, E.E. and M.R. Segura Campos, Neuroprotective effect from Salvia hispanica peptide fractions on pro-inflammatory modulation of HMC3 microglial cells. J Food Biochem, 2020. 44(6): p. e13207.

50. Cappoli, N., et al., The mTOR kinase inhibitor rapamycin enhances the expression and release of pro-inflammatory cytokine interleukin 6 modulating the activation of human microglial cells. EXCLI J, 2019. 18: p. 779-798. 


\section{Supplementary information}

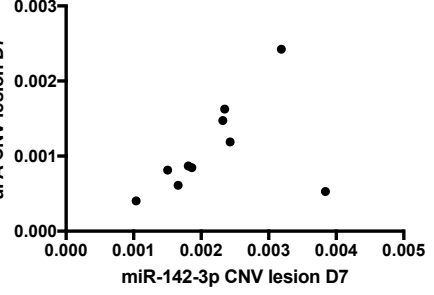

\section{Supplementary Figure 1.}

Spearman correlation between uPA level and miR-142-3p level in CNV lesion 7 days post laser induction $(\mathrm{r}=$ 0.503: $\mathrm{p}=0.144)$.

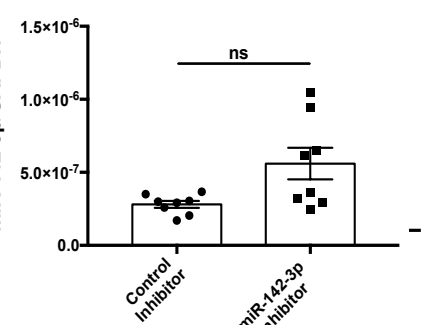

\section{Supplementary Figure 2.}

Expression of miR-142-3p was measured in CNV mouse retinas 7 days post laser-induction and intravitreal injection of miR-142-3p inhibitor and relative negative control $(\mathrm{n}=8$ per experimental group) $(\mathrm{ns}=$ not significant).

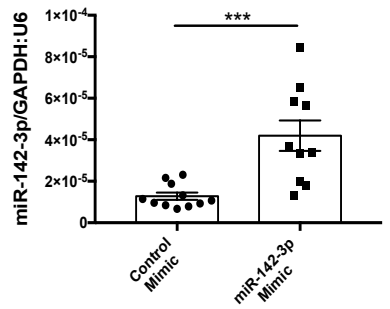

\section{Supplementary Figure 3.}

Expression of miR-142-3p was measured in CNV mouse retinas 7 days post laser-induction and intravitreal injection of miR-142-3p mimic and relative negative control $(n=10-11$ per experimental group) $(* * *=p \leq$ $0.001)$. 


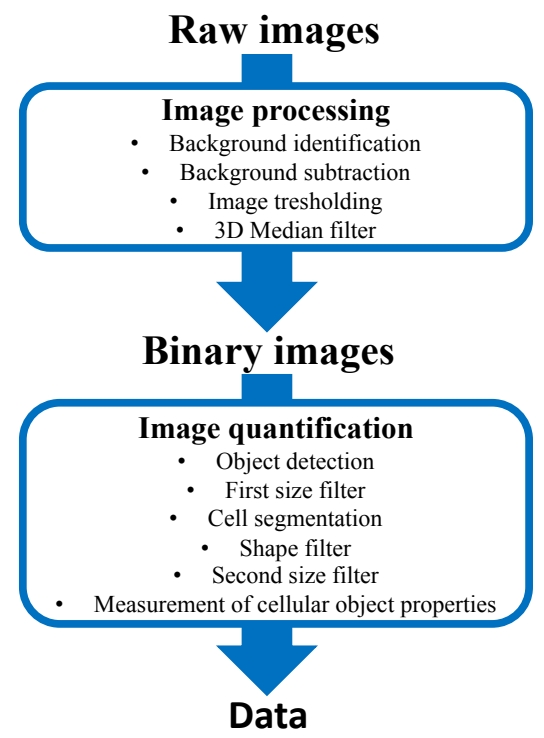

Supplementary Figure 4.

Workflow chart of image processing and quantification for microglia solidity analysis on flat-mounted retinas. 
Chapter 5: General Discussion 


\section{MicroRNA implication in AMD and DR}

The aims of this thesis were: 1) to evaluate the potential of ZSF1 rat as a model of diabetic retinopathy, 2) to identify dysregulated microRNAs in animal models of DR and AMD and 3) to explore whether the modulation of promising microRNA candidates identified under 2) were able to influence choroidal neovascularization.

In Chapter 3, we demonstrate that ZSF1 rat model, despite showing precursor signs of DR within retinal vasculature, failed to trigger further DR progression. This resistance to retinopathy development prompted us to further decipher its underlying genetic regulation. We point out a set of potential protective genes (i.e. Neuropeptide Y and several crystallin genes) overexpressed in obese rats that might be responsible of ZSF1 rat resistance to DR development. In Chapter 4, we define a set of 5 angiogenesis and inflammation-related microRNAs whose expression was upregulated in both human vitreous of diabetic patients and retinas of Akimba mice, a model of advanced DR. We suggest that these microRNAs might be functional regulators of DR progression. Finally, in Chapter 5, we show that miR$142-3 p$ is a functional mediator of choroidal neovascularization, the hallmark of wet AMD. Intravitreal injections of miR-142-3p inhibitor and mimic in the laser-induced CNV mouse model highlighted the pro-angiogenic and pro-inflammatory effects of this poorly described microRNA. We particularly demonstrate a clear link between miR-142-3p and microglia activation, both in vivo and in vitro.

Taken together, AMD and DR are responsible for the majority of blindness cases worldwide and are thus a major public health issue [1]. Often neglected, eye health is however crucial for maintaining a normal life. Half of the population consider losing vision as the worst possible health outcome [2]. Because no cure for AMD and DR is available, prevention remains the best strategy to avoid detrimental sight decrease. Thus, lowering AMD and DR risk by quitting or (best) not starting smoking, practicing regular exercise, having a healthy diet and controlling diabetes are the main everyday life modifiable risk factors [3-5]. AMD and DR show distinct pathophysiology but share biological features, namely inflammation and angiogenesis. This results in similar current treatment strategy for advanced AMD and DR, i.e. intravitreal injections of anti-VEGF drugs. Despite having been a revolution in AMD treatment a couple decades ago and reducing blindness incidence in AMD patients [6], anti-VEGF therapy now shows its limitations and an urgent clinical need for new therapies has emerged [7]. Moreover, repeated intravitreal injections are often painful and/or stressful for patients [8] and anti-VEGF drugs, despite showing discrepancies between molecules, are often quite expensive [9]. These medical, psychological and economic impacts should lead to the development of new drugs. While several clinical trials assessing microRNAs as targets are currently under process for different cancer types, no microRNA entered yet a clinical trial for ocular pathologies (Table 1) [10, 11]. With increasing evidence of microRNA functionality in eye diseases, opportunities for clinical management via microRNA are great and promising. 


\begin{tabular}{|c|c|c|c|c|}
\hline miRNA target & $\begin{array}{l}\text { Drug } \\
\text { name }\end{array}$ & $\begin{array}{c}\text { Type of } \\
\text { miRNA drug }\end{array}$ & Disease & $\begin{array}{c}\text { Development } \\
\text { status }\end{array}$ \\
\hline miR-16 & MesomiR-1 & $\begin{array}{l}\text { TargomiR } \\
\text { (Mimic) }\end{array}$ & $\begin{array}{l}\text { Mesothelioma/ } \\
\text { Lung cancer }\end{array}$ & Phase I \\
\hline miR-21 & $\begin{array}{c}\text { Lademirsen } \\
\text { (RG-012) }\end{array}$ & Inhibitor & Alport Syndrome & Phase I \\
\hline miR-29 & $\begin{array}{c}\text { Remlarsen } \\
\text { (MRG-201) }\end{array}$ & Mimic & Fibrous scar formation & Phase II \\
\hline miR-34 & MRX34 & Mimic & Primary liver cancer & Phase I \\
\hline miR-92 & MRG-110 & LNA inhibitor & Wound healing & Phase I \\
\hline miR-122 & Miravirsen & LNA inhibitor & Hepatitis $\mathrm{C}$ virus & Phase I/II \\
\hline miR-155 & $\begin{array}{l}\text { Cobomarsen } \\
\text { (MRG-106) }\end{array}$ & LNA inhibitor & $\begin{array}{c}\text { Cutaneous T-cell } \\
\text { lymphoma }\end{array}$ & Phase II \\
\hline
\end{tabular}

Table 1: microRNA therapeutic candidates in interventional clinical trial 


\section{Limitations of the study \& perspectives}

With this work, we provide evidence for the use of ZSF1 rat model as a model of resistance to DR onset, as well as the use of microRNAs as promising therapeutic targets in different ocular pathologies. However, several perspectives might be considered for further investigations and deeper characterization. Also, several technical limitations have to be considered in regard to the interpretation of the presented results.

Regarding the ZSF1 rat model, we were able to highlight several genes that might be responsible for the apparent resistance to DR development. First, including more phenotypic readouts for DR onset such as histology on retinal sections, counting of pericyte cells (instead of pericyte coverage), microglia morphology analysis (instead of only number) and lightsheet microscopy of full eyeballs may be of valuable interest to have an overall view of ZSF1 rat retina phenotype. While we investigated gene dysregulation in ZSF1 rats, we did not consider a microRNA analysis. Having a microRNA expression analysis on ZSF1 rat retinas could help us to further understand the surprising lack of DR phenotype in this diabetic animal model. We could expect that some microRNAs upregulated in the Akimba mice might be downregulated in ZSF1 rats. Thus, these microRNAs, upregulated in model of DR and downregulated in model of resistance to DR, might have functional implications in retinopathy onset. It would be of particular interest to analyze the expression pattern of miR142-3p over age in ZSF1 rats. Moreover, we analyzed gene expression only at the first and latest ages, but having a more precise view by analyzing intermediate timepoints could also bring some answers. Finally, the difference that we observed in arteriolar tortuosity and Crys genes family may eventually be obesity related and thus linked to the leptin receptor mutation status of lean and obese rats. 
Regarding the human vitreous and Akimba mouse model study, several perspectives might be considered.

First, increasing the cohort of diabetic patients and enlarging it by recruiting DR patients would be necessary to conclude of microRNAs expression in human samples of DR. Indeed, we were able to conduct our qRT-PCR screening only on a low number of human samples, and thus might not sufficiently reflect human pathology. This low number of samples may also explain why only upregulated microRNAs were highlighted while no significant downregulation was observed. Individual variability was consequent in regards to microRNA expression, in both healthy and diabetic groups. Increasing patient cohort may reduce this unwanted variability. Moreover, collecting other types of human samples, such as blood or post-mortem eyeballs, would be of particular interest. Regular access to blood samples from healthy controls and DR patients during their clinical follow-up would allow to evaluate how microRNA expression changes in response to treatment. Collecting fresh post-mortem human eyeballs could allow us to evaluate microRNA expression in the retina and eventually choroid of DR patients.

In a second time, assessment of microRNA function in the Akimba mice by intravitreal or systemic injections of microRNA inhibitors and mimics would be of particular interest. Despite we showed microRNA dysregulation in Akimba retinas, we did not bring any proof of functionality. Thus, in vivo microRNA modulation might be considered for further characterization. Also, assessing Crys and Npy genes expression in Akimba mice and parental strains may allow to further confirm their implication in DR onset. However, the Akimba mouse being under a patent protection, it is hardly accessible for academic purposes. To overcome the technical limitation of Akimba accessibility, the use of another DR animal model may be considered. Using the well-established and easily manageable STZ-induced model may be relevant. Moreover, this model mainly reflecting the early DR stage, and thus mainly the inflammatory processes, it could be useful to better determine whether miR-142$3 \mathrm{p}$ is more inflammation or angiogenesis-related. Finally, evaluating in vitro diverse cellular functions, such as proliferation, migration and phagocytosis activity of microglia cells by mimicking DR onset with high or low glucose culture conditions, or by modulating the expression of most promising microRNAs could help to decipher the tight regulation between DR and microRNAs. 
Regarding the role of miR-142-3p during CNV progression, we provide first evidence of this microRNA as a therapeutic target for AMD. However, specific microglia activation should be first further confirmed and then, mechanistic information on microglia activation by miR-142-3p might be needed.

To address the first question, a deeper molecular analysis of microglia may be performed, both in vivo and in vitro. In the laser-induced CNV model, the extended use of microglia-specific markers such as TMEM19 or P2Y12 should be considered in order to allow a clear discrimination between resident microglia and infiltrating macrophages. Combining the use of classical Iba1 marker and CD45 expression profile may also be considered. Despite being relevant, morphological analysis of microglia activation may be combined with molecular approaches. Thus, evaluating expression levels of different surface markers such as CD14 and CD16, specific to M1 activated microglia, from retinal isolated microglia may further confirm miR-142-3p implication in microglia activation. In vitro, the same markers should be used to confirm microglia activation, on both primary microglia and well-established cell line such as HMC3 (human) and BV2 (murine) cells.

To address the mechanistic information, several perspectives may be considered. Thus, in vitro assessment of microglia proliferation, migration, phagocytosis activity and cytokines production under modulation of miR-142-3p could help us to better understand the way this microRNA is able to regulate inflammation and angiogenesis in vivo. Interactions between endothelial cells and microglia cells should also be studied since miR-142-3p acts at both vascular and inflammatory sides. Moreover, despite showing a clear link between miR-142$3 p$ expression and microglia activation, we did not show the cellular source of miR-142-3p. To do so, in situ hybridizations could help in determining which cell type produces miR-1423 p. However, being a really low abundant microRNA, in situ hybridization are technically challenging. Also, despite showing an effect of BCLAF1 expression, a previously described miR-142-3p target, we do not show any functional direct target of miR-142-3p that might be responsible of microglia activation and decreased vascular phenotype. Cross analysis of RNA-sequencing data from laser-induced CNV mouse model and target databases (such as TargetScan and miRTarBase) might highlight target genes whose function could be linked to observed phenotypes. Optimization of inhibitor and mimic concentration for intra-vitreal injections, bioavailability as well as diffusion of these molecules within the mouse eye should be considered. Despite we observed an effect by injecting these molecules with no carrier, including the molecule in exosomes or other type of vesicles may increase tissue penetration and thus efficiency.

Finally, despite showing a beneficial effect of miR-142-3p inhibition on CNV progression, its efficiency should be compared to current standard of care treatments (i.e. Lucentis, Avastin, Eylea or Beovu) for actual preclinical proof of concept. 


\section{Concluding remarks}

The data presented in this thesis take us a step closer to an understanding on the implication and contribution of microRNAs in diabetic retinopathy and age-related macular degeneration. It appears clear from this doctoral work that microRNAs regulate several ocular pathologies and might be considered as targets for new clinical therapeutic strategies. However, the use of a miR-targeting strategy in the clinic implies a deeper characterization and actual molecular regulation by microRNAs of such diseases, as well as safety analysis, target optimization and pharmaco-kinetics and dynamics studies. MicroRNAs having pleiotropic functions on diverse genes sets, these tiny molecules should be considered as double-edged swords: great clinical applications come along with potential harmful sideeffects. Overall, this work could be a resource to fuel future investigations on targeting microRNAs in ocular pathologies. 


\section{References}

1. Flaxman, S.R., et al., Global causes of blindness and distance vision impairment 1990-2020: a systematic review and meta-analysis. The Lancet Global Health, 2017. 5(12): p. e1221-e1234.

2. $\quad$ Scott, A.W., et al., Public Attitudes About Eye and Vision Health. JAMA Ophthalmol, 2016. 134(10): p. 1111-1118.

3. Makin, R.D., et al., Voluntary Exercise Suppresses Choroidal Neovascularization in Mice. Invest Ophthalmol Vis Sci, 2020. 61(5): p. 52.

4. Guymer, R.H. and E.W. Chong, Modifiable risk factors for age-related macular degeneration. Med J Aust, 2006. 184(9): p. 455-8.

5. Tyrberg, M., et al., Overweight, hyperglycemia and tobacco use are modifiable risk factors for onset of retinopathy 9 and 17years after the diagnosis of diabetes A retrospective observational nation-wide cohort study. Diabetes Res Clin Pract, 2017. 133: p. 21-29.

6. Finger, R.P., et al., Anti-vascular endothelial growth factor in neovascular agerelated macular degeneration - a systematic review of the impact of anti-VEGF on patient outcomes and healthcare systems. BMC Ophthalmol, 2020. 20(1): p. 294.

7. Zuber-Laskawiec, K., et al., Non-responsiveness and tachyphylaxis to antivascular endothelial growth factor treatment in naive patients with exudative agerelated macular degeneration. J Physiol Pharmacol, 2019. 70(5).

8. Senra, H., et al., Psychological impact of anti-VEGF treatments for wet macular degeneration-a review. Graefes Arch Clin Exp Ophthalmol, 2016. 254(10): p. 1873-1880.

9. van Asten, F., et al., The cost-effectiveness of bevacizumab, ranibizumab and aflibercept for the treatment of age-related macular degeneration-A costeffectiveness analysis from a societal perspective. PLoS One, 2018. 13(5): p. e0197670.

10. Hanna, J., G.S. Hossain, and J. Kocerha, The Potential for microRNA Therapeutics and Clinical Research. Front Genet, 2019. 10: p. 478.

11. Jones, D., Setbacks shadow microRNA therapies in the clinic. Nat Biotechnol, 2018. 36(10): p. 909-910. 

Addendum 


\section{Summary}

"The eye as a miRror: targeting microRNAs in ocular pathologies" is a doctoral work performed by Quentin Roblain from February 2017 to January 2021 in collaboration between the University of Liège, Belgium (under the supervision of Prof. Dr. Agnès Noël) and Maastricht University, The Netherlands (under the supervision of Prof. Dr. Stephane Heymans). The main goal of this doctoral work was to evaluate the biological implication of microRNAs during age-related macular degeneration (AMD) and diabetic retinopathy (DR).

Vision impairment and blindness affect 2.2 billion individuals worldwide. Despite causing no direct death, vision impairment increases by a factor 3 the risk of being involved in a vehicle collision, the likelihood of suffering from depression and being unemployed. It also doubles the risk of having a fall while walking. AMD and DR are two vision-threatening eye disorders which are currently treated by intravitreal delivery of anti-VEGF drugs. However, patients exhibit great discrepancies in response to this treatment, and no cure for AMD and DR are available. MicroRNAs, a class of non-coding RNAs, have diverse biological functions during development, normal physiology and pathophysiology. These molecules could thus eventually be promising therapeutic targets in eye pathologies. In this work, the biological implication of microRNAs during AMD and DR was evaluated. In Chapter 1, every notion needed for a comprehensive reading of subsequent chapters is introduced, reviewed and summarized, microRNAs being the common thread between sections. Thus, a focus is made on their biology, their implication in the developing and adult eye, as well as in the pathological eye and finally, their role in microglia cells. In Chapter 2, the suitability of ZSF1 rat model as a model of DR is evaluated. Despite showing hyperglycemia an increased arteriolar tortuosity, the ZSF1 rats failed to develop further diabetic retinopathy. A potential genes network is next highlighted, possibly preventing ZSF1 rats from developing DR. In Chapter 3, an expression profile of microRNAs in human and mouse samples and their potential as biomarker or therapeutic target in DR is made and discussed. In Chapter 4, specific overexpression of miR-142-3p is revealed in CNV lesion of a mouse model of choroidal neovascularization. Implication of miR-142-3p in activation of microglia cells both in vitro and in vivo is next demonstrated. Finally, in Chapter 5, a global discussion of the present work is made, as well as suggestions of perspectives that might be considered for a better understanding of microRNAs implication in AMD and DR.

In conclusion, this doctoral work presents biological contributions of microRNAs in two eye pathologies, namely age-related macular degeneration and diabetic retinopathy. These findings extend the current knowledge on AMD and DR pathophysiology, and may contribute to the development of novel therapeutic strategies. 


\section{Valorization}

- Socio-economic relevance

Vision impairment and blindness affect 2.2 billion people worldwide and, in Europe, its estimated cost is 25.83 billion euros. Moreover, besides their large contribution to the global healthcare system cost, vision impairment and blindness can have a profound psychological impact on affected individuals, as well as on their quality of life. The use of available costeffective treatment and prevention strategies may significantly reduce both financial and social burdens. However, developing more efficient and less expensive drugs may also help with reducing vision impairment and blindness contribution to public health. In particular, despite a great progress in AMD and DR treatment since the approval of anti-VEGF therapy, these two retinal diseases are still not curable. Thus, a better understanding of AMD and DR biology is needed for the development of innovative drugs. In this regard, a close collaboration between basic scientists and clinicians is required, and will in the end be beneficial for patients.

\section{- $\quad$ Target groups}

The content of this doctoral work is broadly relevant for the scientific and academic communities. It may be of particular interest for other scientists working in the field of experimental ophthalmology, as well as for any other researcher whose area of study includes microRNAs. Since miR-142-3p implication in inflammation and microglia activation has been demonstrated in this doctoral work, it may also interest any scientists focused on other inflammatory or neurodegenerative diseases, such as Alzheimer's disease. In a larger extent, it may also be of particular interest for biopharmaceutical companies whose are aiming to develop new DR and AMD drugs.

\section{- Innovation}

This project highlights the potential of microRNAs as therapeutic targets in the context of two highly prevalent ocular diseases, namely age-related macular degeneration and diabetic retinopathy. Therefore, implementing specific microRNA inhibitors and/or mimics for the treatment of ocular diseases in a patent protection could be relevant if stronger proof of efficiency is provided.

\section{- Future directives}

Translation of the findings of this doctoral work to the clinic will be the most challenging goal for future directives. A big gap exists between basic research and clinical research, and future studies will have to build the bridge between them by addressing the questions of AMD and DR molecular regulation by microRNAs, as well as the safety, target optimization and pharmaco-kinetics and dynamics of the use of oligonucleotide molecules as therapeutic agents. 


\section{Acknowledgements}

Doing a PhD is like a twisty adventure: you know the start and the finale, but you don't have any idea of the path in-between. In the end, it makes you stronger, more mature and responsible. Hopefully, you are not alone for this adventure! I would like to acknowledge here all the people that helped me, as well as all the people that I met during this exciting adventure. Thank you all!

I gratefully acknowledge Agnès and Stephane for giving me the opportunity to realize my $\mathrm{PhD}$ under their joint supervision. Agnès, merci pour ta disponibilité, pour ta complaisance et pour ta bienveillance. Stephane, bedankt voor je advies, voor je dynamiek, en voor je hulp. I also want to acknowledge both of you for your help for the revisions of the different chapters of this thesis.

I also gratefully acknowledge Ingrid Struman, Paula da Costa Martins, Lieve Moons, Sebastien Foulquier, Theo Gorgels, Bernard Duchesne and Bernard Mari, the members of assessment and defense committees, for the critical reading of this manuscript. Dear Ingrid and Paula, thanks a lot for your help and advice during my annual committees.

Julie, tu auras été véritablement l'initiatrice de ce projet scientifique. Pour ta pédagogie, pour tes conseils éclairés, pour ta constante disponibilité, pour ton dynamisme à toute épreuve et pour m'avoir donné l'opportunité de mener à bien ce projet avec toi, je tiens à vivement te remercier ! Merci également pour ta bonne humeur quotidienne et la confiance dont tu as fait preuve envers moi au cours de ma thèse.

Merci Géraldine pour tout ce temps passé ensemble. Depuis les événements du CEB jusqu'à notre super bureau décoré avec goût, en passant par plein de soirées, de nombreux kilomètres de jogging et quelques petites blagues (pas) marrantes, une vraie amitié s'est installée entre nous. Partager le quotidien de la thèse avec toi pendant 4 ans fut un réel plaisir. Je te souhaite tout le meilleur pour la suite de ta carrière, et je sais que de nombreux kilomètres de course sont encore devant nous!

Coucou petite perruche ! Merci Cassandre, pour ton aide pratique, pour tes conseils avisés, pour le partage de ton savoir et de ton expérience, ainsi que pour ton soutien moral. Merci également pour tous les bons moments passés au labo et en dehors, et pour la complicité qui s'est installée entre nous. Je suis convaincu que quelle que soit ta future carrière, académique ou non, tu trouveras ton épanouissement professionnel, et je te souhaite le meilleur pour la suite !

Merci Adrien, Coline et Alice, mes autres collègues de bureau, avec qui j'ai eu le plaisir de partager le quotidien pendant quelques semaines, mois ou années ! Ce quotidien fut partagé entre moments de réflexion scientifique, de confidences, de fous rire, et de soutien moral, 
ainsi que de blagues entre collègues et de vengeances qui s'en suivent. Merci à vous trois pour tous ces bons moments.

Dear Ilona, my Maastricht and Leuven colleague and friend, thank you so much for your sympathy, for your kindness and for your help! I wish we could have worked more together during our PhDs. I wish you the best of luck with your new career and hope to see you soon.

Merci Vincent pour ton aide au cours de mes expériences, pour tes conseils, pour ta gentillesse, pour ta bonne humeur et pour tes super blagues. Avec Géraldine, vous faisiez vraiment la paire ! Ta disponibilité constante, même lorsque tu es retourné à la banque des yeux, m'aura bien aidé au cours de ma thèse.

Dear Vincenza, thank you for your help at the really beginning of my thesis. I learnt a lot working with you! I wish you good luck in your own career.

Merci Thomas, Lionel, Medhi et Pierre, mes partenaires de labo. Merci pour les moments passés au labo, mais surtout pour les après-midis jeux de société, les soirées de gala (parfois un peu trop) arrosées, les conversations un peu loufoques et tous les autres bons moments. Merci Anne (Annichou pour les intimes), Aline (mon autre partenaire de jogging), Marie, Marine, Charles, Laetitia et Carmen, mes autres partenaires de labo. Merci pour tous les moments passés ensemble, que ce soit au labo ou à l'extérieur. Les différentes activités du Télévie, les joggings et autres activités plus sportives, les repas chez l'un et chez l'autre et tous ces bon moments. Merci Anthony, Alizée, Isabelle B, Ekaterina, Alison, MarieLaure et Perrine. A vous tous, merci pour la super convivialité que vous avez apporté au quotidien. L'aventure au LBTD n'aurait pas été aussi agréable sans votre présence !

Merci Tania, Christel S, Florent, Natacha, Marie-Émilie, Justine, Céline H, Anna-Lisa, Irina, Oriane, Maureen, Maïté, Laure et Charline, les anciennes et anciens du labo. Nos chemins se sont croisés plus ou moins longtemps, et j'en garde un très bon souvenir !

Merci Fabrice, Sébastien, Loïc, Amélie, Isabelle DDS, Florence, Vincent, Daphnée et Sheila, les plus nouveaux arrivés au sein de l'équipe du LBTD. J'espère que vous apprécierez tout autant que moi votre passage au sein de cette grande équipe et je compte sur vous pour assurer une bonne ambiance !

Merci Nathalie, Erika, Émilie \& Isabelle D, la super équipe technique du LBTD ! Je suis très reconnaissant pour votre aide, votre pédagogie et votre disponibilité pour le travail quotidien. Merci Fabienne, Céline V \& Damien, l'autre super équipe technique du LBTD. Dear Rick \& Wouter, thank you for your technical help in the lab. Merci Hélène \& Karin, l'équipe administrative du LBTD, sans qui le travail quotidien serait rendu nettement plus compliqué et plus fastidieux. Dear Barbara \& Lilian, thank you for your kind help! Even remotely, I always get rapid answer to any of my questions, whatever the matter. 
Dear Paolo, thank you so much for your kind and precious help in Leuven! I learnt a lot working with you during animal experiments. I wish you all the best for your $\mathrm{PhD}$, and hope to see you soon. Dear Emma, you unfortunately arrived in Maastricht a few weeks after I left for Liège. I wish we could have work more together, and I really hope you appreciate your new position in Denver. Dear Annika \& Michiel, thank you for being nice colleagues, and good luck for your PhD. Merci Sandrine, ma partenaire des pauses café et ma collègue francophone. J'espère que nous pourrons bientôt retourner boire un café ensemble, cela fait tellement longtemps! Dear Robin, Monika, Steffie \& Leni, my former Maastricht colleagues, thank you for your help and best of luck with your respective career.

Merci Christel P, Christine, Carine, Erik, Nor Edine, les PIs du LBTD. Vos conseils et votre aide lors des différentes réunions et discussions scientifiques aura été précieuse. Dear Blanche \& Marc, thank you for your help and your advice.

Merci Louis, Pierre \& Lindsey, mes stagiaires que j'ai eu la chance de pouvoir de superviser au cours de cette thèse. Je vous souhaite tout le meilleur pour la suite. Louis, je suis sûr que ton travail et ta persévérance seront payants, et je te souhaite de pouvoir réaliser ta thèse au sein du LBTD.

Merci Alex V, pour m'avoir donné l'envie d'entreprendre un doctorat. Je te souhaite tout le meilleur du coté de Boston, et me réjouis de te revoir ! Merci Geoffrey, Julien, Delphine \& Catherine, mes précieux amis. Petit à petit, nos chemins commencent à se séparer de plus en plus, mais je suis convaincu que le temps ne ternira pas notre amitié. A tous ces moments passés ensemble, et à ceux à venir ! Merci Céline R, d'avoir été là dans les meilleurs moments, comme dans les moins bons. Puisse notre amitié durer aussi longtemps que possible ! Merci Marie S, d'avoir aussi été là dans les meilleurs et les moins bons moments. Je suis convaincu que tu feras une brillante carrière!

Il me tient également particulièrement à cœur de remercier ma famille proche. Merci Nanou, Thibaut, Papa \& Maman. Merci pour votre soutien, vos encouragements et votre foi en moi. Merci Maman, toi qui a toujours été là pour moi et qui t'es dévouée pour tes deux enfants.

Finalement, tout ce travail n'aurait pas été possible sans le soutien constant et sans faille de mon compagnon de vie. Merci Nathan, toi qui a toujours trouvé les mots et les gestes pour m'épauler, me rassurer, me conseiller et me supporter. Nous avons déjà parcouru un petit bout de chemin ensemble, et j'espère que cette aventure-là, elle, ne prendra jamais fin. Je t'aime. 


\section{Curriculum Vitae}

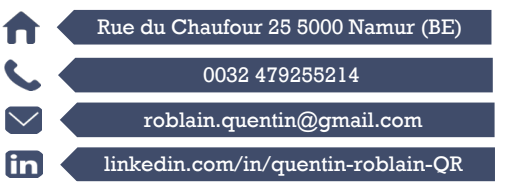

\section{Quentin Roblain}

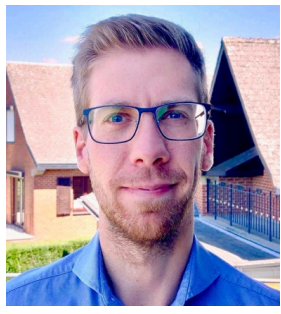

Early stage researcher with strong aptitudes for multidisciplinary work. Familiar with pre-clinical research, clinical trial, intellectual property and eye pathologies. Autonomous, polyvalent and leadership. Looking for a great job opportunity in the biomedical field, and within a dynamic and emerging group.

\section{Education Work experiences}

- Double PhD (Biomedical and Pharmaceutical Sciences)

2017 - Present | ULiège \& UMaastricht

- Master in Biochemistry and Molecular and Cellular Biology

2014-2016 | ULiège

- Bachelor in Biological Sciences 2010-2014 | ULiège

\section{Professional skills}

- Project management

- Clinical trial (certificate in progress)

- Translational medicine

- Molecular and cell culture techniques

- Animal research (FELASA C)

\section{Soft skills}

- Conflict resolution

- Decision making

- Solving problem

- Communication/vulgarization

Languages

- French: native

- English: fluent

- Dutch: basics

\section{- PhD candidate}

University of Liège (BE), Biology of Tumors and Development Lab \& Maastricht University (NL), Heart Failure Research Lab

2017 - Present

Exploring microRNAs in experimental ophthalmology

- Volunteer for COVID-19 federal task force University of Liège (BE) 2020

Virus inactivation step

\section{- Education animator for guided tour} Aquarium-Museum of Liège (BE)

2012-2017

Animation for children and/or adult groups

- Intern researcher in developmental biology University of Liège (BE) - Protein Signalling and Interactions Lab

2016

Angiogenesis in zebrafish

- Intern researcher in plant physiology University of Liège (BE) - Functional Genomics Lab 2016

Metal hyperaccumulation in plants

\section{- Intern researcher in bioengineering} CEBEDEAU (Liège, BE)

2015

Wastewater treatment via activated granular sludge

\section{Referees}

- Prof. Dr. Agnès NOËL Agnes.Noel@uliege.be

- Prof. Dr. Stephane HEYMANS s.heymans@maastrichtuniversity.nl 